\title{
UHPLC-ESI-QTOF-MS/MS-Based Molecular Networking Guided Isolation and Dereplication of Antibacterial and Antifungal Constituents of Ventilago denticulata
}

\author{
Muhaiminatul Azizah 1미 Patcharee Pripdeevech 2,3, Tawatchai Thongkongkaew ${ }^{1}$, \\ Chulabhorn Mahidol ${ }^{1,4}$, Somsak Ruchirawat ${ }^{1,4,5}$ and Prasat Kittakoop 1,4,5,*(D) \\ 1 Chulabhorn Graduate Institute, Chemical Biology Program, Chulabhorn Royal Academy, \\ Laksi, Bangkok 10210, Thailand; mimi.hufflepuf@gmail.com (M.A.); tawatchait@cgi.ac.th (T.T.); \\ mahidol_natlab@cri.or.th (C.M.); somsak@cri.or.th (S.R.) \\ 2 School of Science, Mae Fah Luang University, Muang, Chiang Rai 57100, Thailand; patcharee.pri@mfu.ac.th \\ 3 Center of Chemical Innovation for Sustainability (CIS), Mae Fah Luang University, \\ Muang, Chiang Rai 57100, Thailand \\ 4 Chulabhorn Research Institute, Kamphaeng Phet 6 Road, Laksi, Bangkok 10210, Thailand \\ 5 Center of Excellence on Environmental Health and Toxicology (EHT), CHE, Ministry of Education, \\ Bangkok 10210, Thailand \\ * Correspondence: prasat@cri.or.th; Tel.: +66-869755777
}

Received: 6 August 2020; Accepted: 12 September 2020; Published: 15 September 2020

\begin{abstract}
Ventilago denticulata is an herbal medicine for the treatment of wound infection; therefore this plant may rich in antibacterial agents. UHPLC-ESI-QTOF-MS/MS-Based molecular networking guided isolation and dereplication led to the identification of antibacterial and antifungal agents in $V$. denticulata. Nine antimicrobial agents in $V$. denticulata were isolated and characterized; they are divided into four groups including (I) flavonoid glycosides, rhamnazin 3-rhamninoside (7), catharticin or rhamnocitrin 3-rhamninoside (8), xanthorhamnin B or rhamnetin 3-rhamninoside (9), kaempferol 3-rhamninoside (10) and flavovilloside or quercetin 3-rhamninoside (11), (II) benzisochromanquinone, ventilatones B (12) and A (15), (III) a naphthopyrone ventilatone C (16) and (IV) a triterpene lupeol (13). Among the isolated compounds, ventilatone $C$ (16) was a new compound. Moreover, kaempferol, chrysoeriol, isopimpinellin, rhamnetin, luteolin, emodin, rhamnocitrin, ventilagodenin A, rhamnazin and mukurozidiol, were tentatively identified as antimicrobial compounds in extracts of $V$. denticulata by a dereplication method. MS fragmentation of rhamnose-containing compounds gave an oxonium ion, $\mathrm{C}_{6} \mathrm{H}_{9} \mathrm{O}_{3}{ }^{+}$at $m / z 129$, while that of galactose-containing glycosides provided the fragment ion at $m / z 163$ of $\mathrm{C}_{6} \mathrm{H}_{11} \mathrm{O}_{5}{ }^{+}$. These fragment ions may be used to confirm the presence of rhamnose or galactose in mass spectrometry-based analysis of natural glycosides or oligosaccharide attached to biomolecules, that is, glycoproteins.
\end{abstract}

Keywords: Ventilago denticulata; natural products; antibacterial activity; antifungal activity; dereplication; molecular networking; flavonoid glycosides; mass spectrometry; MS fragmentation of sugar

\section{Introduction}

Natural products are important sources of drugs and they provide many building blocks for drug discovery [1]. Statistically, around 50\% of approved drugs were derived from natural products [2]. From 1931 to 2013, new chemical entities from natural products approved by the US Food and Drug 
Administration (FDA), are approximately $47 \%$ derived from plants, followed by $30 \%$ from bacteria, $23 \%$ from fungi and $5 \%$ from other natural sources [3]. As per the World Health Organisation (WHO), approximately $65 \%$ of the population of the world particularly in developing countries, mostly rely on utilization of plant-derived traditional medicines for health care and ethnomedical-based treatments [4]. Furthermore, in 2015, Youyou Tu received the Nobel Prize award for the discovery of artemisinin as an anti-malarial drug from the plant Artemisia annua; this underscores the importance of plant metabolites as sources of modern drugs [2]. According to these data, plants are rich sources of bioactive compounds, contributing significantly to drug discovery.

A conventional approach for drug discovery from natural products takes long time and high cost with hard efforts in purification, isolation and identification of natural products [5]. Moreover, the end of this process may result in the rediscovery of known bioactive compounds [6,7]. To increase the rate of the discovery of new natural products, dereplication technique is an alternative approach. Dereplication enables the identification of known compounds and the potential unknown compounds in crude extracts at the early stage of research before the isolation process [8]. The dereplication technique employs liquid chromatography-mass spectrometry(LC-MS), liquid chromatography-photodiode array detector (LC-PDA), liquid chromatography-nuclear magnetic resonance (LC-NMR) or other spectroscopic techniques [5,6] and LC-MS provides high sensitivity and effectiveness for the identification of natural products [6].

There is a limitation for LC-MS based dereplication using parent masses because it yields several molecular formulas when searching in databases [9]; this leads to less effectiveness for compound identification. Since compounds with similar structures tend to have similar MS/MS fragmentation patterns, information from MS/MS data of chemical similarity is used for molecular networking, which is considered as an effective dereplication strategy [6]. MS/MS-based molecular networking emerges as a new technique to supplement the dereplication strategy [10]. The Global Natural Products Social Molecular Networking (GNPS) website (http://gnps.ucsd.edu) is an open-access web-based mass spectrometry, facilitating high-throughput online dereplication and molecular networking analysis [11]. At present, molecular networking has been successfully employed to discover new bioactive compounds from natural sources such as the discovery of penicanesones A-C from Penicillium canescens and selaginpulvilins M-T from Selaginella tamariscina [12,13]. MS/MS-based molecular networking is involved in an untargeted fragmentation study of all compounds in crude extracts, the MS/MS spectra alignment and assembling the spectra into nodes in the network based on spectral similarity [10]. The result from MS/MS-based molecular networking is the relational networks, which reveal relationship and distribution of each chemical constituent presented in crude extracts $[12,13]$.

Antibiotic resistance has been a public health problem worldwide. By 2050, it is predicted that death because of infection of antibiotic-resistant strains will reach approximately 10 million people per year [14]. Hence, the research on the discovery of novel antibiotics is needed. Ventilago denticulata Willd. is a plant in the family Rhamnaceae; previously it was named Ventilago calyculata. In Thailand, V. denticulata is called "Thao-Wan-Lek" or "Rhang-Dang." Interestingly, in the West Midnapore district of West Bengal, the Eastern State of India, the plant $V$. denticulata is widely used to treat wound infection, suggesting the presence of antibacterial agents in this plant [15]. Bacterial strains found in wound infection were $37 \%$ of Staphylococcus aureus, $17 \%$ of Pseudomonas aeruginosa and $6 \%$ of Escherichia coli [16]. Bacteria, Bacillus cereus and Salmonella enterica serovar Typhimurium, were also found in wound infection from immunocompetent patients or diabetes mellitus patients $[17,18]$. Candida albicans was the most widely detected fungus in wound infection especially in diabetic foot ulcers [19]. Therefore, this research aims to explore antibacterial and antifungal agents in V. denticulata. Previously, a crude bark extract of $V$. denticulata was reported to show the antibacterial and antifungal activities [20,21]. Our previous work revealed that $V$. denticulata had a few antibacterial agents [22]. Based on these studies, $V$. denticulata could be a potential source of medicinally useful compounds, especially antimicrobial and antifungal agents. This work explores antibacterial agents in crude 
extracts and fractions of $V$. denticulata using UHPLC-ESI-QTOF-MS/MS analysis, as well as a molecular networking. It is known that different parts of plants may have different chemical constituents and thus exerting different pharmacological effects [23]. We report herein antibacterial and antifungal compounds in both bark and trunk of a plant, V. denticulata.

\section{Results and Discussions}

\subsection{Dereplication of Compounds from Crude Extracts of V. denticulata and Guided Isolation by} UHPLC-ESI-QTOF-MS/MS-Based Molecular Networking

Fresh trunk and bark of $V$. denticulata were sequentially extracted with methanol $(\mathrm{MeOH})$ and dichloromethane $\left(\mathrm{CH}_{2} \mathrm{Cl}_{2}\right)$. Both $\mathrm{MeOH}$ and $\mathrm{CH}_{2} \mathrm{Cl}_{2}$ crude extracts were analyzed by UHPLC-ESI-QTOF-MS/MS. In this research, there were two scan types; first, LC-MS scans a total ion chromatogram (TIC) and base peak chromatogram (BPC). Both positive and negative MS ionization modes were performed because some classes of compounds such as sesquiterpenes and thiophenes were well-detected in a positive ionization mode, whereas flavonoids, phenolic acids and quinic acid could be detected by a negative ion mode [24]. Besides, the mechanism of fragmentation of positive and negative ion modes was dissimilar and they may afford supplementary structural information [25]. Overlay of TIC chromatograms of $\mathrm{MeOH}$ and $\mathrm{CH}_{2} \mathrm{Cl}_{2}$ crude extracts of $V$. denticulata is shown in Figure S1, Supplementary Materials. Second, auto-MS ${ }^{2}$ was performed in which the most predominant $\mathrm{MS}^{1}$ ions are chosen for $\mathrm{MS}^{2}$ fragmentation. From MS/MS spectra, the chemical constituents in crude extracts of $V$. denticulata were tentatively identified; they are listed in Table 1. The putative known and unknown compounds were annotated by the Agilent MassHunter METLIN Metabolomics Database, the Human Metabolome Database (https://hmdb.ca/) and online database Metlin (http://metlin.scripps.edu/index.php), as well as by comparison with standard compounds. The present work has seven standard compounds including (+)- $R$-ventilagolin, emodin, rutin, naringenin, 6-hydroxy flavone, chrysin and (+)-catechin.

As shown in Table 1, several compounds in crude extracts were identified in either positive or negative ionization mode. There are 93 tentatively identified compounds listed in Table 1; these metabolites have been reported as plant metabolites. Among the compounds identified in Table 1, emodin, physcion, ventilagodenin $\mathrm{A}$ and $(+)-(R)$-ventilagolin previously isolated by our group [22] were indeed found in crude extracts of $V$. denticulata and they underwent MS/MS fragmentation in both positive and negative ionization modes. We performed further analysis using the GNPS website; all acquired MS/MS data were converted into MzXML as an open file format by ProteoWizard. Then, the converted data were uploaded to create molecular networking on the GNPS website (http://gnps.ucsd.edu). All molecular networking data obtained from the GNPS system were imported to Cytoscape 3.7.2 version, in order to visualize and simplify molecular networking in one display. The node colors were set and they represented MS/MS data of compounds present in crude extracts or standard compounds. Cytoscape was used for rapid analysis of the whole profile of metabolites in all crude extracts, as well as for the correlation between standard compounds and their analogs. Result of the molecular networking of crude extracts in a positive mode is shown in Figure 1a, while that of a negative ionization mode is in Supplementary data (Figure S2); they are used as a complementary method for the dereplication strategy. 
Table 1. Tentatively identified compounds in the bark and trunk of V. denticulata obtained from LC-MS/MS analysis. Compounds identified by Metlin Database [M], Human Metabolome Database [H] and standard compounds [S].

\begin{tabular}{|c|c|c|c|c|c|c|c|c|c|c|}
\hline No. & $\underset{(\min )}{t_{R}}$ & Compounds & $\begin{array}{l}\text { Molecular } \\
\text { Formula }\end{array}$ & Mass & Adduct Ions & $\begin{array}{c}\text { Observed } \\
m / z\end{array}$ & $\begin{array}{c}\text { Calculated } \\
m / z\end{array}$ & $\underset{(\mathrm{ppm})}{\Delta}$ & Fragment Ions $(m / z)$ & $\begin{array}{l}\text { Found in } \\
\text { Extracts }{ }^{a}\end{array}$ \\
\hline 1 & 1.086 & Unidentified & $\mathrm{C}_{18} \mathrm{H}_{3} \mathrm{NO}_{14}$ & 487.1895 & $(\mathrm{M}-\mathrm{H})^{-}$ & 486.1826 & 486.1828 & 0.50 & $\begin{array}{c}\text { 341.1082, } 179.0561, \\
144.0663,119.0346 \\
101.0242\end{array}$ & DT \\
\hline 2 & 1.091 & Unidentified & $\mathrm{C}_{37} \mathrm{H}_{36} \mathrm{~N}_{2} \mathrm{O}_{11}$ & 684.2316 & $(\mathrm{M}-\mathrm{H})^{-}$ & 683.2244 & 683.2246 & 0.40 & $\begin{array}{c}\text { 341.1086, } 179.0556 \\
119.0346\end{array}$ & $\mathrm{MB}, \mathrm{MT}$ \\
\hline 3 & 1.357 & $\begin{array}{c}\text { 2'-Methoxy-3-(2,4-dihydroxyphenyl) } \\
\text {-1,2-propanediol 4'-glucoside } \\
{[\mathrm{M}, \mathrm{H}]}\end{array}$ & $\mathrm{C}_{16} \mathrm{H}_{24} \mathrm{O}_{9}$ & 360.1420 & $(\mathrm{M}+\mathrm{Na})^{+}$ & 383.1313 & 383.1313 & -0.01 & $\begin{array}{c}306.9908,248.9974 \\
207.0666,185.0403 \\
102.0900\end{array}$ & $\mathrm{MB}, \mathrm{MT}$ \\
\hline \multirow[t]{2}{*}{4} & \multirow[t]{2}{*}{1.371} & \multirow[t]{2}{*}{ Kaempferol-3-rhamninoside [M] } & \multirow[t]{2}{*}{$\mathrm{C}_{33} \mathrm{H}_{40} \mathrm{O}_{19}$} & \multirow[t]{2}{*}{740.2162} & $(\mathrm{M}+\mathrm{H})^{+}$ & 741.2233 & 741.2237 & 0.50 & $\begin{array}{c}595.1677,449.1072 \\
346.0867,287.0557 \\
147.0649\end{array}$ & MB \\
\hline & & & & & $(\mathrm{M}-\mathrm{H})^{-}$ & 739.2080 & 739.2091 & 1.49 & $\begin{array}{l}285.0396,255.0315 \\
479.1186,317.0657\end{array}$ & MB, MT \\
\hline 5 & 1.405 & Rhamnetin 3-rhamninoside $[\mathrm{M}]$ & $\mathrm{C}_{34} \mathrm{H}_{42} \mathrm{O}_{20}$ & 770.2269 & $(\mathrm{M}+\mathrm{H})^{+}$ & 771.2343 & 771.2342 & -0.09 & $\begin{array}{l}239.0928,163.0602, \\
147.0653,129.0548\end{array}$ & MB, MT \\
\hline \multirow[t]{2}{*}{6} & \multirow[t]{2}{*}{1.414} & \multirow[t]{2}{*}{$\begin{array}{c}\text { 1,2,10-Trihydroxydihydro-trans-linalyl oxide } \\
\text { 7-O- } \beta \text {-D-glucopyranoside }[\mathrm{M}, \mathrm{H}]\end{array}$} & \multirow[t]{2}{*}{$\mathrm{C}_{16} \mathrm{H}_{30} \mathrm{O}_{10}$} & \multirow[t]{2}{*}{382.1841} & $\begin{array}{c}(\mathrm{M}-\mathrm{H})^{-} \\
(\mathrm{M}+\mathrm{Na})^{+}\end{array}$ & $\begin{array}{l}769.2192 \\
405.1733\end{array}$ & $\begin{array}{l}769.2197 \\
405.1731\end{array}$ & $\begin{array}{c}0.66 \\
-0.54\end{array}$ & $\begin{array}{c}315.0505,299.0186 \\
355,0125,273.1298 \\
129.0543\end{array}$ & $\begin{array}{c}\mathrm{MB}, \mathrm{MT}, \mathrm{DT} \\
\mathrm{MB}\end{array}$ \\
\hline & & & & & $(\mathrm{M}-\mathrm{H})^{-}$ & 381.1762 & 381.1766 & 1.15 & $\begin{array}{l}\text { 322.0691, 249.1343, } \\
\text { 205.3362, 161.0450, } \\
113.0235,101.0243\end{array}$ & $\mathrm{MB}, \mathrm{DB}$ \\
\hline \multirow[t]{2}{*}{7} & \multirow[t]{2}{*}{1.493} & \multirow[t]{2}{*}{ Rhamnocitrin 3- rhamninoside [M] } & \multirow[t]{2}{*}{$\mathrm{C}_{34} \mathrm{H}_{42} \mathrm{O}_{19}$} & \multirow[t]{2}{*}{754.2320} & $(\mathrm{M}+\mathrm{H})^{+}$ & 755.2394 & 755.2393 & -0.19 & $\begin{array}{c}463.1233,301.0709 \\
163.0600,147.0651 \\
129.0543\end{array}$ & MB, MT, DT \\
\hline & & & & & $(\mathrm{M}-\mathrm{H})^{-}$ & 753.2239 & 753.2248 & 1.12 & $\begin{array}{c}557.2233,299.0554 \\
283.0236 \\
647.1279,575.1043\end{array}$ & $\mathrm{MB}, \mathrm{MT}, \mathrm{DT}$ \\
\hline 8 & 1.499 & Unidentified & $\mathrm{C}_{27} \mathrm{H}_{34} \mathrm{~N}_{7} \mathrm{O}_{21}$ & 792.1803 & $(\mathrm{M}+2 \mathrm{H})^{+2}$ & 397.0973 & 397.0977 & 0.97 & $\begin{array}{l}545.1010,501.0683 \\
399.0395,339.0179, \\
201.0041,121.0495\end{array}$ & $\mathrm{MB}, \mathrm{MT}$ \\
\hline \multirow[t]{2}{*}{9} & \multirow[t]{2}{*}{1.535} & \multirow[t]{2}{*}{ Furocoumarinic acid glucoside $[\mathrm{M}, \mathrm{H}]$} & \multirow[t]{2}{*}{$\mathrm{C}_{17} \mathrm{H}_{18} \mathrm{O}_{9}$} & \multirow[t]{2}{*}{366.0955} & $(\mathrm{M}+\mathrm{H})^{+}$ & 367.1024 & 367.1024 & -0.21 & $\begin{array}{l}349.0928,331.0806, \\
307.0803,289.0703, \\
275.0556,263.0559, \\
217.0494,161.0594\end{array}$ & DB \\
\hline & & & & & $(\mathrm{M}-\mathrm{H})^{-}$ & 365.0871 & 365.0878 & 1.96 & $\begin{array}{l}350.0639,306.0746, \\
289.0707,274.0482, \\
246.0522,161.0181\end{array}$ & DB \\
\hline 10 & 1.540 & Unidentified & $\mathrm{C}_{27} \mathrm{H}_{50} \mathrm{Cl}_{2} \mathrm{~N}_{9} \mathrm{O}_{8} \mathrm{~S}$ & 762.2601 & $(\mathrm{M}+\mathrm{Na})^{+}$ & 785.2493 & 785.2493 & -0.03 & $\begin{array}{c}\text { 493.1342, } 331.0815 \\
147.0664\end{array}$ & DB \\
\hline
\end{tabular}


Table 1. Cont

\begin{tabular}{|c|c|c|c|c|c|c|c|c|c|c|}
\hline No. & $\begin{array}{c}t_{\mathrm{R}} \\
(\mathrm{min})\end{array}$ & Compounds & $\begin{array}{l}\text { Molecular } \\
\text { Formula }\end{array}$ & Mass & Adduct Ions & $\begin{array}{c}\text { Observed } \\
m / z\end{array}$ & $\begin{array}{c}\text { Calculated } \\
m / z\end{array}$ & $\begin{array}{c}\Delta \\
(\mathrm{ppm})\end{array}$ & Fragment Ions $(m / z)$ & $\begin{array}{l}\text { Found in } \\
\text { Extracts }\end{array}$ \\
\hline 11 & 1.547 & Unidentified & $\mathrm{C}_{29} \mathrm{H}_{30} \mathrm{~N}_{16} \mathrm{O}_{11}$ & 778.2272 & $(\mathrm{M}+2 \mathrm{H})^{+2}$ & 412.1027 & 412.1032 & 1.32 & $\begin{array}{c}677.1394,575.1073 \\
429.0485,369.0279 \\
266.0451,201.0073 \\
129.0543\end{array}$ & MB \\
\hline 12 & 1.558 & $\begin{array}{c}\text { 3, } 3^{\prime}, 4^{\prime} \text {-Trihydroxyflavone } \\
\text { 3-O-[ } \alpha \text {-L-rhamnopyranosyl- }(1 \rightarrow 2) \\
{[\alpha-\mathrm{L}-\text {-rhamnopyranosyl- }(1 \rightarrow 6)} \\
\text { ]- } \beta \text {-D-glucopyranoside }] \\
{[\mathrm{M}, \mathrm{H}]}\end{array}$ & $\mathrm{C}_{33} \mathrm{H}_{40} \mathrm{O}_{18}$ & 724.2206 & $\left(\mathrm{M}+\mathrm{CH}_{3} \mathrm{COO}\right)^{-}$ & 783.2345 & 783.2353 & 1.06 & $\begin{array}{c}453.1600,329.0657 \\
314.0425,145.0503 \\
101.0246\end{array}$ & MB, MT, DB, DT \\
\hline 13 & 1.564 & 5,7,8-Trihydroxyflavanone 7-glucoside [M, H] & $\mathrm{C}_{21} \mathrm{H}_{22} \mathrm{O}_{10}$ & 434.1212 & $(\mathrm{M}-\mathrm{H})^{-}$ & 433.1138 & 433.1140 & 0.59 & $\begin{array}{c}\text { 313.0719, 271.0556, } \\
270.0528,231.0611 \\
139.0402\end{array}$ & MB, DT \\
\hline \multirow[t]{2}{*}{14} & 1.575 & $\begin{array}{c}\text { Rhamnazin 3-rhamninoside } \\
{[\mathrm{M}]}\end{array}$ & $\mathrm{C}_{35} \mathrm{H}_{44} \mathrm{O}_{20}$ & 784.2430 & $(\mathrm{M}+\mathrm{H})^{+}$ & 785.2503 & 785.2503 & 0.00 & $\begin{array}{c}\text { 493.1341, 331.0814, } \\
163.0599,147.0653 \\
129.054\end{array}$ & MB, MT, DB, DT \\
\hline & & & & & $(\mathrm{M}-\mathrm{H})^{-}$ & 783.2349 & 783.2348 & -0.13 & $\begin{array}{c}537.1992,453.1584 \\
329.0664,234.1049 \\
145.0490\end{array}$ & MB, MT, DB, DT \\
\hline 15 & 1.626 & Astragalin $[\mathrm{M}, \mathrm{H}]$ & $\mathrm{C}_{21} \mathrm{H}_{20} \mathrm{O}_{11}$ & 448.1009 & $(\mathrm{M}+\mathrm{H})^{+}$ & 449.1081 & 449.1078 & -0.58 & $\begin{array}{l}317.0661,287.0553 \\
269.0444,195.0657\end{array}$ & $\mathrm{MB}, \mathrm{MT}, \mathrm{DT}$ \\
\hline 16 & 1.629 & Unidentified & $\mathrm{C}_{23} \mathrm{H}_{30} \mathrm{~N}_{7} \mathrm{O}_{8}$ & 532.2155 & $(\mathrm{M}+\mathrm{Na})^{+}$ & 555.2043 & 555.2048 & 0.88 & $\begin{array}{l}381.1307,286.0742, \\
207.0619,147.0433 \\
346.8297 .327 .0481\end{array}$ & MB \\
\hline 17 & 1.634 & Kaempferol 5-glucoside [M, H] & $\mathrm{C}_{21} \mathrm{H}_{20} \mathrm{O}_{11}$ & 448.0996 & $(\mathrm{M}+\mathrm{HCOO})^{-}$ & 493.0979 & 493.0988 & 1.76 & $\begin{array}{c}298.0487,285.0402 \\
240.0460\end{array}$ & $\mathrm{MB}, \mathrm{MT}, \mathrm{DT}$ \\
\hline 18 & 1.635 & Naringenin $4^{\prime}$-O-glucuronide $[\mathrm{M}, \mathrm{H}]$ & $\mathrm{C}_{21} \mathrm{H}_{20} \mathrm{O}_{11}$ & 448.1002 & $(\mathrm{M}+\mathrm{Na})^{+}$ & 471.0895 & 471.0898 & 0.57 & $\begin{array}{c}339.0471,309.0368 \\
294.0188,249.1094 \\
161.9958\end{array}$ & $\mathrm{MB}, \mathrm{MT}$ \\
\hline \multirow[t]{2}{*}{19} & 1.708 & Aloesol $[\mathrm{M}, \mathrm{H}]$ & $\mathrm{C}_{13} \mathrm{H}_{14} \mathrm{O}_{4}$ & 234.0891 & $(\mathrm{M}+\mathrm{H})^{+}$ & 235.0964 & 235.0965 & 0.45 & $\begin{array}{l}\text { 217.0860, 191.0705, } \\
\text { 163.0754, 151.0385, } \\
135.0804,107.0847\end{array}$ & MB, MT, DT \\
\hline & & & & & $(\mathrm{M}-\mathrm{H})^{-}$ & 233.0818 & 233.1819 & 0.60 & $\begin{array}{c}\text { 189.0552, } 161.0593 \\
149.0251\end{array}$ & $\mathrm{MB}, \mathrm{MT}, \mathrm{DB}$ \\
\hline 20 & 1.765 & Zingerone glucoside $[\mathrm{M}, \mathrm{H}]$ & $\mathrm{C}_{17} \mathrm{H}_{24} \mathrm{O}_{8}$ & 356.1468 & $(\mathrm{M}+\mathrm{Na})^{+}$ & 379.1361 & 379.1363 & 0.63 & $\begin{array}{c}\text { 323.9212, 278.3414, } \\
\text { 235. 8741, 217.0847, } \\
111.0775\end{array}$ & $\mathrm{MB}, \mathrm{DB}$ \\
\hline 21 & 1.810 & Unidentified & $\mathrm{C}_{33} \mathrm{H}_{46} \mathrm{~N}_{4} \mathrm{O}_{6}$ & 594.3419 & $(\mathrm{M}+\mathrm{H})^{+}$ & 595.3492 & 595.349 & -0.35 & $\begin{array}{l}577.3542,536.2739 \\
173.1640,120.0805\end{array}$ & MB \\
\hline 22 & 1.852 & Unidentified & $\mathrm{C}_{21} \mathrm{H}_{28} \mathrm{O}_{8}$ & 408.1771 & $(\mathrm{M}+\mathrm{Na})^{+}$ & 431.1674 & 431.1676 & 0.53 & $\begin{array}{c}317.1031,275.0908 \\
205.0465\end{array}$ & DB \\
\hline
\end{tabular}


Table 1. Cont

\begin{tabular}{|c|c|c|c|c|c|c|c|c|c|c|}
\hline No. & $\begin{array}{c}t_{R} \\
(\min )\end{array}$ & Compounds & $\begin{array}{l}\text { Molecular } \\
\text { Formula }\end{array}$ & Mass & Adduct Ions & $\begin{array}{c}\text { Observed } \\
m / z\end{array}$ & $\begin{array}{c}\text { Calculated } \\
\mathrm{m} / \mathrm{z}\end{array}$ & $\begin{array}{c}\Delta \\
(\mathrm{ppm})\end{array}$ & Fragment Ions $(m / z)$ & $\begin{array}{l}\text { Found in } \\
\text { Extracts a }\end{array}$ \\
\hline \multirow[t]{2}{*}{23} & 1.909 & Xanthotoxol glucoside $[\mathrm{M}, \mathrm{H}]$ & $\mathrm{C}_{17} \mathrm{H}_{16} \mathrm{O}_{9}$ & 364.0795 & $(\mathrm{M}+\mathrm{H})^{+}$ & 365.0866 & 365.0867 & 0.36 & $\begin{array}{l}305.0661,291.0851, \\
277.0713,259.0606, \\
215.0704,132.0900\end{array}$ & DB \\
\hline & & & & & $(\mathrm{M}-\mathrm{H})^{-}$ & 363.0716 & 363.0722 & 1.51 & $\begin{array}{c}304.0583,287.0556, \\
272.0320,261.0404, \\
244.0375,228.0435 \\
201.0195\end{array}$ & DB \\
\hline 24 & 2.000 & Unidentified & $\mathrm{C}_{24} \mathrm{H}_{18} \mathrm{~N}_{8} \mathrm{O}_{4}$ & 482.1448 & $(\mathrm{M}+\mathrm{Na})^{+}$ & 505.1338 & 505.1343 & 0.96 & $\begin{array}{l}419.1317,343.1048, \\
257.0809,127.0393 \\
335.0877,257.0804\end{array}$ & MB \\
\hline \multirow[t]{2}{*}{25} & 2.006 & Isoliquiritin $[\mathrm{M}, \mathrm{H}]$ & $\mathrm{C}_{21} \mathrm{H}_{22} \mathrm{O}_{9}$ & 418.1261 & $(\mathrm{M}+\mathrm{H})^{+}$ & 419.1334 & 419.1337 & 0.71 & $\begin{array}{c}239.0703,191.0702 \\
127.0390\end{array}$ & MB \\
\hline & & & & & $(\mathrm{M}-\mathrm{H})^{-}$ & 417.1184 & 417.1191 & 1.66 & $297.0764,255.0643$ & $\mathrm{MB}, \mathrm{MT}, \mathrm{DB}$ \\
\hline 26 & 2.042 & 6"-O-Acetyldaidzin [M, H] & $\mathrm{C}_{23} \mathrm{H}_{22} \mathrm{O}_{10}$ & 458.1209 & $(\mathrm{M}+\mathrm{HCOO})^{-}$ & 503.1192 & 503.1195 & 0.67 & $\begin{array}{c}\text { 418.1190, 297.0765, } \\
255.0690\end{array}$ & MB \\
\hline 27 & 2.190 & Glucoemodin $[\mathrm{M}, \mathrm{H}]$ & $\mathrm{C}_{21} \mathrm{H}_{20} \mathrm{O}_{10}$ & 432.1051 & $(\mathrm{M}-\mathrm{H})^{-}$ & 431.0979 & 431.0984 & 1.07 & $\begin{array}{l}344.8229,311.0557, \\
269.0448,227.1067 \\
359.0953,246.0522,\end{array}$ & MB, MT, DT \\
\hline 28 & 2.282 & Kievitol [M, H] & $\mathrm{C}_{20} \mathrm{H}_{22} \mathrm{O}_{7}$ & 374.1356 & $(\mathrm{M}-\mathrm{H})^{-}$ & 373.1284 & 373.1293 & 2.29 & $\begin{array}{c}193.0504,179.0714, \\
164.0475,149.0600 \\
134.0368\end{array}$ & DB \\
\hline \multirow[t]{2}{*}{29} & 2.291 & Wharangin $[\mathrm{M}, \mathrm{H}]$ & $\mathrm{C}_{17} \mathrm{H}_{12} \mathrm{O}_{8}$ & 344.0536 & $(\mathrm{M}+\mathrm{H})^{+}$ & 345.0608 & 345.0605 & -0.74 & $\begin{array}{l}\text { 303.0497, 327.0487, } \\
299.0544,275.0543, \\
261.0401,195.0290\end{array}$ & $\mathrm{DB}, \mathrm{DT}$ \\
\hline & & & & & $(\mathrm{M}-\mathrm{H})^{-}$ & 343.0454 & 343.0459 & 1.69 & $\begin{array}{c}330.0381,301.0348 \\
287.0196,273.0040 \\
158.0608 \\
517.8187,458.3673\end{array}$ & DB \\
\hline 30 & 2.314 & $\begin{array}{c}\text { 4"-Methyl-6"-(3,4-dihydroxy-E-cinnamoyl) } \\
\text { isoorientin }[\mathrm{M}, \mathrm{H}]\end{array}$ & $\mathrm{C}_{31} \mathrm{H}_{28} \mathrm{O}_{14}$ & 624.1474 & $(\mathrm{M}-\mathrm{H})^{-}$ & 623.1400 & 623.1406 & 1.02 & $\begin{array}{c}375.3759,298.0471, \\
295.0808,285.0416, \\
241.0516\end{array}$ & MB \\
\hline 31 & 2.376 & Chrysoeriol $[\mathrm{M}, \mathrm{H}]$ & $\mathrm{C}_{16} \mathrm{H}_{12} \mathrm{O}_{6}$ & 300.0640 & $(\mathrm{M}+\mathrm{H})^{+}$ & 301.0712 & 301.0707 & -1.80 & $\begin{array}{c}273.0397,260.0310 \\
255.0651,245.0442 \\
227.0698 \\
270.0168,258.0166\end{array}$ & DB \\
\hline & & & & & $(\mathrm{M}-\mathrm{H})^{-}$ & 299.0561 & 299.0561 & 0.00 & $\begin{array}{c}255.0661,240.0428, \\
227.0346,214.0269, \\
151.0033\end{array}$ & DB \\
\hline 32 & 2.377 & 6"-Malonylcosmosiin [M, H] & $\mathrm{C}_{24} \mathrm{H}_{22} \mathrm{O}_{13}$ & 518.1048 & $(\mathrm{M}-\mathrm{H})^{-}$ & 517.0975 & 517.0988 & 2.44 & $\begin{array}{c}473.1078,432.1734 \\
385.1729,269.0452 \\
225.0402\end{array}$ & MB \\
\hline
\end{tabular}


Table 1. Cont.

\begin{tabular}{|c|c|c|c|c|c|c|c|c|c|c|}
\hline No. & $\begin{array}{c}t_{\mathrm{R}} \\
(\mathrm{min})\end{array}$ & Compounds & $\begin{array}{l}\text { Molecular } \\
\text { Formula }\end{array}$ & Mass & Adduct Ions & $\begin{array}{c}\text { Observed } \\
m / z\end{array}$ & $\begin{array}{c}\text { Calculated } \\
m / z\end{array}$ & $\begin{array}{c}\Delta \\
(\mathrm{ppm})\end{array}$ & Fragment Ions $(\mathrm{m} / \mathrm{z})$ & $\begin{array}{l}\text { Found in } \\
\text { Extracts }{ }^{\mathrm{a}}\end{array}$ \\
\hline 33 & 2.382 & Cicerin 7-(6-malonylglucoside) [M, H] & $\mathrm{C}_{26} \mathrm{H}_{26} \mathrm{O}_{15}$ & 578.1269 & $(\mathrm{M}+\mathrm{H})^{+}$ & 579.1341 & 579.1344 & 0.64 & $\begin{array}{c}437.0247,342.9891 \\
331.0819,147.0531 \\
127.0390\end{array}$ & MB \\
\hline 34 & 2.416 & Unidentified & $\mathrm{C}_{40} \mathrm{H}_{38} \mathrm{NO}_{5} \mathrm{~S}$ & 644.2467 & $(\mathrm{M}+\mathrm{Na})^{+}$ & 667.2356 & 667.2363 & 1.04 & $\begin{array}{l}553.2780,425.0864 \\
329.1411,129.0528\end{array}$ & MB, DT \\
\hline \multirow[t]{2}{*}{35} & 2.445 & Quercetin $[\mathrm{M}, \mathrm{H}]$ & $\mathrm{C}_{15} \mathrm{H}_{10} \mathrm{O}_{7}$ & 302.0422 & $(\mathrm{M}+\mathrm{H})^{+}$ & 303.0494 & 303.0499 & 1.86 & $\begin{array}{l}276.8345,240.8436, \\
229.0471,195.0268, \\
182.9751,139.8692\end{array}$ & MB \\
\hline & & & & & $(\mathrm{M}-\mathrm{H})^{-}$ & 301.0351 & 301.0354 & 0.76 & $\begin{array}{l}273.0382,229.0518, \\
178.9980,151.0032, \\
121.0300,107.0132 \\
537.1811,410.0280\end{array}$ & MB \\
\hline \multirow[t]{2}{*}{36} & 2.552 & Unidentified & $\mathrm{C}_{18} \mathrm{H}_{40} \mathrm{~N}_{5} \mathrm{O}_{18}$ & 614.2359 & $(\mathrm{M}+\mathrm{Na})^{+}$ & 637.2247 & 637.2261 & 2.19 & $\begin{array}{c}339.1044,145.0475 \\
110.0979\end{array}$ & MB, DT \\
\hline & & & & & $(\mathrm{M}-\mathrm{H})^{-}$ & 349.0924 & 349.0929 & 1.35 & $\begin{array}{l}\text { 334.0694, 319.0457, } \\
291.0506,219.0304 \\
242.0590,217.0500,\end{array}$ & DB \\
\hline \multirow[t]{2}{*}{37} & 2.563 & Emodinanthranol $[\mathrm{M}, \mathrm{H}]$ & $\mathrm{C}_{15} \mathrm{H}_{12} \mathrm{O}_{4}$ & 256.0738 & $(\mathrm{M}+\mathrm{H})^{+}$ & 257.0810 & 257.0808 & -0.66 & $\begin{array}{l}214.0612,198.9313, \\
145.0656,101.0594\end{array}$ & DB \\
\hline & & & & & $(\mathrm{M}-\mathrm{H})^{-}$ & 255.0658 & 255.0663 & 1.84 & $\begin{array}{c}213.0555,187.0768 \\
183.0814\end{array}$ & DB \\
\hline 38 & 2.753 & $\alpha$-Hydrojuglone 4-O- $\beta$-D-glucoside [H] & $\mathrm{C}_{13} \mathrm{H}_{18} \mathrm{O}_{5}$ & 338.0995 & $(\mathrm{M}-\mathrm{H})^{-}$ & 337.0922 & 337.0929 & 1.98 & $\begin{array}{c}250.0844,221.081 \\
163.0765\end{array}$ & $\mathrm{MB}, \mathrm{DB}, \mathrm{DT}$ \\
\hline 39 & 3.039 & Unidentified & $\mathrm{C}_{13} \mathrm{H}_{20} \mathrm{~N}_{3} \mathrm{O}_{8} \mathrm{~S}$ & 378.0958 & $(\mathrm{M}+\mathrm{H})^{+}$ & 379.1025 & 379.1044 & 4.86 & $\begin{array}{l}319.0809,291.0861, \\
202.0630,111.0421 \\
333.0949,301.0702\end{array}$ & DB \\
\hline 40 & 3.087 & Unidentified & $\mathrm{C}_{17} \mathrm{H}_{18} \mathrm{O}_{8}$ & 350.1003 & $(\mathrm{M}+\mathrm{H})^{+}$ & 351.1075 & 351.1074 & -0.19 & $\begin{array}{c}276.0630,259.0604 \\
215.0700\end{array}$ & DB \\
\hline 41 & 3.131 & Unidentified & $\mathrm{C}_{28} \mathrm{H}_{24} \mathrm{O}_{12}$ & 552.1264 & $(\mathrm{M}-2 \mathrm{H})^{-2}$ & 275.0558 & 275.0561 & 1.17 & $\begin{array}{c}338.0072,262.0703, \\
232.0368,218.0236, \\
188.0462\end{array}$ & DB \\
\hline 42 & 3.233 & Isopimpinellin $[\mathrm{M}, \mathrm{H}]$ & $\mathrm{C}_{13} \mathrm{H}_{10} \mathrm{O}_{5}$ & 246.0522 & $\left(\mathrm{M}+\mathrm{CH}_{3} \mathrm{COO}\right)^{-}$ & 305.0660 & 305.0667 & 2.34 & $\begin{array}{l}\text { 245.0447, 201.0512, } \\
173.0585,129.0714\end{array}$ & DB \\
\hline 43 & 3.274 & Kaempferol $[\mathrm{M}, \mathrm{H}]$ & $\mathrm{C}_{15} \mathrm{H}_{10} \mathrm{O}_{6}$ & 286.0480 & $(\mathrm{M}+\mathrm{H})^{+}$ & 287.0554 & 287.0550 & -1.39 & $\begin{array}{l}227.8855,165.0174 \\
153.0172,121.0271 \\
257.0426,241.0493\end{array}$ & $\mathrm{MB}, \mathrm{MT}$ \\
\hline & & & & & $(\mathrm{M}-\mathrm{H})^{-}$ & 285.0401 & 285.0405 & 1.37 & $\begin{array}{c}229.0487,211.0396 \\
151.0029\end{array}$ & $\mathrm{MB}, \mathrm{MT}, \mathrm{DB}$ \\
\hline 44 & 3.291 & Coriandrone $\mathrm{C}[\mathrm{M}, \mathrm{H}]$ & $\mathrm{C}_{13} \mathrm{H}_{10} \mathrm{O}_{5}$ & 246.0536 & $(\mathrm{M}+\mathrm{H})^{+}$ & 247.0609 & 247.0601 & -3.39 & $\begin{array}{l}229.0499,219.0262, \\
201.0552,173.0586, \\
158.0695,137.1239\end{array}$ & DB \\
\hline
\end{tabular}


Table 1. Cont.

\begin{tabular}{|c|c|c|c|c|c|c|c|c|c|c|}
\hline No. & $\begin{array}{c}t_{R} \\
(\min )\end{array}$ & Compounds & $\begin{array}{l}\text { Molecular } \\
\text { Formula }\end{array}$ & Mass & Adduct Ions & $\begin{array}{c}\text { Observed } \\
m / z\end{array}$ & $\begin{array}{l}\text { Calculated } \\
m / z\end{array}$ & $\begin{array}{c}\Delta \\
(\mathrm{ppm})\end{array}$ & Fragment Ions $(m / z)$ & $\begin{array}{l}\text { Found in } \\
\text { Extracts }{ }^{a}\end{array}$ \\
\hline \multirow[t]{2}{*}{45} & 3.364 & Eriodictyol $[\mathrm{M}, \mathrm{H}]$ & $\mathrm{C}_{15} \mathrm{H}_{12} \mathrm{O}_{6}$ & 288.0631 & $(\mathrm{M}-\mathrm{H})^{-}$ & 289.0704 & 289.0707 & 0.84 & $\begin{array}{l}\text { 271.0589, 259.0603, } \\
257.0465,231.0641, \\
229.0488,173.0582\end{array}$ & DB \\
\hline & & & & & $(\mathrm{M}-\mathrm{H})^{-}$ & 287.0558 & 287.0561 & 0.93 & $\begin{array}{l}\text { 259.0604, 243.0653, } \\
201.0582,177.0550 \\
151.0041,125.0243 \\
243.1493,241.0487\end{array}$ & $\mathrm{MB}, \mathrm{MT}, \mathrm{DT}$ \\
\hline 46 & 3.583 & Coumesterol $[\mathrm{M}, \mathrm{H}]$ & $\mathrm{C}_{15} \mathrm{H}_{8} \mathrm{O}_{5}$ & 268.0373 & $(\mathrm{M}+\mathrm{H})^{+}$ & 269.0446 & 269.0444 & -0.46 & $\begin{array}{l}213.0553,185.0602 \\
157.0644\end{array}$ & $\mathrm{MB}, \mathrm{MT}$ \\
\hline \multirow[t]{2}{*}{47} & 3.594 & Citreorosein $[\mathrm{M}, \mathrm{H}]$ & $\mathrm{C}_{15} \mathrm{H}_{10} \mathrm{O}_{6}$ & 286.0482 & $(\mathrm{M}+\mathrm{H})^{+}$ & 287.0555 & 287.0550 & -1.65 & $\begin{array}{l}269.0447,213.0536 \\
185.0593\end{array}$ & $\mathrm{MB}, \mathrm{MT}, \mathrm{DT}$ \\
\hline & & & & & $(\mathrm{M}-\mathrm{H})^{-}$ & 285.0404 & 285.0405 & 0.35 & $\begin{array}{l}241.0503,172.9762 \\
257.0808,243.0644\end{array}$ & MB, MT, DB, DT \\
\hline \multirow[t]{2}{*}{48} & 3.608 & Physcion $[\mathrm{M}]$ & $\mathrm{C}_{16} \mathrm{H}_{12} \mathrm{O}_{5}$ & 284.0688 & $(\mathrm{M}+\mathrm{H})^{+}$ & 285.0761 & 285.0757 & -1.4 & $\begin{array}{l}239.0696,229.0496 \\
211.0750\end{array}$ & DB \\
\hline & & & & & $(\mathrm{M}-\mathrm{H})^{-}$ & 283.0612 & 283.0612 & -0.07 & $\begin{array}{c}255.0650,241.0503, \\
239.0703,227.0345, \\
224.0477\end{array}$ & DB \\
\hline 49 & 3.698 & $R$-Angolensin $[\mathrm{M}]$ & $\mathrm{C}_{16} \mathrm{H}_{16} \mathrm{O}_{4}$ & 272.1051 & $(\mathrm{M}+\mathrm{H})^{+}$ & 273.1124 & 273.1121 & -0.81 & $\begin{array}{l}\text { 255.1016, 231.1015, } \\
227.1068,189.0915, \\
174.0667,111.8671\end{array}$ & DB \\
\hline 50 & 3.894 & $( \pm)$-Sphaerosin $[\mathrm{M}, \mathrm{H}]$ & $\mathrm{C}_{17} \mathrm{H}_{18} \mathrm{O}_{5}$ & 302.1153 & $(\mathrm{M}+\mathrm{H})^{+}$ & 303.1225 & 303.1227 & 0.80 & $\begin{array}{l}\text { 285.1117, 261.1129, } \\
\text { 257.1174, 219.1029, } \\
204.0783,163.0361\end{array}$ & $\mathrm{MB}, \mathrm{DB}$ \\
\hline 51 & 3.919 & Unidentified & $\mathrm{C}_{34} \mathrm{H}_{36} \mathrm{O}_{10}$ & 604.2310 & $(\mathrm{M}+\mathrm{Na})^{+}$ & 627.2204 & 627.2201 & -0.58 & $\begin{array}{l}325.1052 \\
2680917.259 .0604\end{array}$ & DB \\
\hline 52 & 3.926 & 3-Hydroxyphloretin [M, H] & $\mathrm{C}_{15} \mathrm{H}_{14} \mathrm{O}_{6}$ & 290.0785 & $(\mathrm{M}+\mathrm{HCOO})^{-}$ & 335.0767 & 335.0772 & 1.67 & $\begin{array}{l}268.0917,259.0604, \\
248.0686,220.0728, \\
205.0504,147.0429\end{array}$ & $\mathrm{MB}, \mathrm{DB}$ \\
\hline 53 & 3.996 & $3^{\prime}, 7$-Dihydroxy-4', 8-dimethoxyisoflavone $[\mathrm{H}]$ & $\mathrm{C}_{17} \mathrm{H}_{14} \mathrm{O}_{6}$ & 314.0785 & $(\mathrm{M}-\mathrm{H})^{-}$ & 313.0712 & 313.0718 & 1.82 & $\begin{array}{l}300.0246,269.0808 \\
254.0571,239.0326\end{array}$ & $\mathrm{MB}, \mathrm{DB}$ \\
\hline 54 & 4.062 & Unidentified & $\mathrm{C}_{19} \mathrm{H}_{22} \mathrm{O}_{10}$ & 410.1214 & $(\mathrm{M}+\mathrm{Na})^{+}$ & 433.1106 & 433.1105 & -0.21 & $\begin{array}{l}401.0840,369.0571 \\
341.0618,250.5698\end{array}$ & MB \\
\hline 55 & 4.064 & Unidentified & $\mathrm{C}_{37} \mathrm{H}_{32} \mathrm{~N}_{3} \mathrm{O}_{15}$ & 758.1834 & $(\mathrm{M}-\mathrm{H})^{-}$ & 757.1760 & 757.1761 & 0.13 & $\begin{array}{c}713.1893,458.1202, \\
410.6138,373.7386, \\
299.7235,254.0514 \\
191.1313\end{array}$ & $\mathrm{MB}, \mathrm{MT}$ \\
\hline 56 & 4.075 & Unidentified & $\mathrm{C}_{25} \mathrm{H}_{30} \mathrm{~N}_{8} \mathrm{O}_{7}$ & 650.1400 & $(\mathrm{M}+\mathrm{H})^{+}$ & 651.1473 & 651.1472 & -0.03 & 337.0683 & DB \\
\hline 57 & 4.132 & $\begin{array}{c}\text { 5,6,7,8-Tetrahydroxy-3', } 4^{\prime} \text {-dimethoxyflavone } \\
{[\mathrm{M}, \mathrm{H}]}\end{array}$ & $\mathrm{C}_{17} \mathrm{H}_{14} \mathrm{O}_{8}$ & 346.0681 & $(\mathrm{M}-\mathrm{H})^{-}$ & 345.0610 & 345.0616 & 1.85 & $\begin{array}{l}331.0413,298.0119 \\
270.0171,242.0246 \\
312.0620,286.0477\end{array}$ & MB \\
\hline 58 & 4.134 & 5-Hydroxy-4',7,8-trimethoxyflavone [M, H] & $\mathrm{C}_{18} \mathrm{H}_{16} \mathrm{O}_{6}$ & 328.0939 & $(\mathrm{M}-\mathrm{H})^{-}$ & 327.0867 & 327.0874 & 2.06 & $\begin{array}{l}\text { 312.0620, 286.0477, } \\
271.0240,268.0732, \\
253.0500,225.0558\end{array}$ & DB \\
\hline
\end{tabular}


Table 1. Cont.

\begin{tabular}{|c|c|c|c|c|c|c|c|c|c|c|}
\hline No. & $\begin{array}{c}t_{R} \\
(\min )\end{array}$ & Compounds & $\begin{array}{l}\text { Molecular } \\
\text { Formula }\end{array}$ & Mass & Adduct Ions & $\begin{array}{c}\text { Observed } \\
m / z\end{array}$ & $\begin{array}{l}\text { Calculated } \\
\quad m / z\end{array}$ & $\begin{array}{c}\Delta \\
(\mathrm{ppm})\end{array}$ & Fragment Ions $(m / z)$ & $\begin{array}{l}\text { Found in } \\
\text { Extracts }{ }^{a}\end{array}$ \\
\hline 59 & 4.178 & Unidentified & $\mathrm{C}_{12} \mathrm{H}_{8} \mathrm{~N}_{5} \mathrm{O}_{6} \mathrm{~S}$ & 350.0196 & $(\mathrm{M}+\mathrm{H})^{+}$ & 351.0265 & 351.0268 & 0.77 & $\begin{array}{l}\text { 297.3586, 261.9442, } \\
\text { 245.8488, 222.0035, } \\
181.0472,135.0783\end{array}$ & $\mathrm{MB}$ \\
\hline 60 & 4.203 & Unidentified & $\mathrm{C}_{34} \mathrm{H}_{24} \mathrm{O}_{12}$ & 624.1270 & $(\mathrm{M}+\mathrm{Na})^{+}$ & 647.1162 & 647.1160 & -0.38 & 335.053 & DB \\
\hline \multirow[t]{2}{*}{61} & 4.251 & $\begin{array}{l}\text { Aloe emodin w-acetate }[\mathrm{M}, \mathrm{H}] \\
\text { or Ventilatone A (isolation) }\end{array}$ & $\mathrm{C}_{17} \mathrm{H}_{12} \mathrm{O}_{6}$ & 312.0636 & $(\mathrm{M}+\mathrm{H})^{+}$ & 313.0706 & 313.0707 & 0.29 & $\begin{array}{c}285.0759,271.0604 \\
243.0659,215.0685 \\
167.8890\end{array}$ & $\mathrm{MB}, \mathrm{DB}$ \\
\hline & & & & & $(\mathrm{M}-\mathrm{H})^{-}$ & 311.0559 & 311.0561 & 0.72 & $\begin{array}{c}297.0393,269.0438 \\
268.0373,253.0140 \\
224.0472\end{array}$ & DB \\
\hline \multirow[t]{2}{*}{62} & 4.532 & Cartorimine $[\mathrm{M}, \mathrm{H}]$ & $\mathrm{C}_{15} \mathrm{H}_{14} \mathrm{O}_{6}$ & 290.0794 & $(\mathrm{M}+\mathrm{Na})^{+}$ & 313.0686 & 313.0683 & -1.23 & $\begin{array}{c}276.9105,212.8751 \\
123.1149\end{array}$ & $\mathrm{MB}, \mathrm{MT}$ \\
\hline & & & & & $(\mathrm{M}-\mathrm{H})^{-}$ & 289.0712 & 289.01718 & 1.81 & $\begin{array}{l}273.0402,259.0239 \\
245.0457,201.0550\end{array}$ & MB, MT, DB \\
\hline \multirow[t]{2}{*}{63} & 4.619 & Rhamnetin [M] & $\mathrm{C}_{16} \mathrm{H}_{12} \mathrm{O}_{7}$ & 316.0581 & $(\mathrm{M}+\mathrm{H})^{+}$ & 317.0654 & 317.0654 & 0.56 & $\begin{array}{l}271.0590,243.0679 \\
167.0342,121.0279\end{array}$ & MB \\
\hline & & & & & $(\mathrm{M}-\mathrm{H})^{-}$ & 315.0505 & 315.0510 & 1.57 & $\begin{array}{l}300.0261,166.0221 \\
121.0293,112.9849 \\
270.0163,257.0450\end{array}$ & $\mathrm{MB}, \mathrm{DB}$ \\
\hline 64 & 4.721 & Luteolin $[\mathrm{M}, \mathrm{H}]$ & $\mathrm{C}_{15} \mathrm{H}_{10} \mathrm{O}_{6}$ & 286.0473 & $(\mathrm{M}-\mathrm{H})^{-}$ & 285.0401 & 285.04005 & 1.34 & $\begin{array}{c}241.0499,213.0526 \\
151.9236\end{array}$ & MB, MT, DB, DT \\
\hline 65 & 4.752 & Unidentified & $\mathrm{C}_{18} \mathrm{H}_{14} \mathrm{O}_{7}$ & 342.0744 & $(\mathrm{M}+\mathrm{Na})^{+}$ & 365.0636 & 365.0632 & -1.14 & $\begin{array}{l}321.0373,305.0419 \\
\quad 156.0637\end{array}$ & DB \\
\hline 66 & 4.874 & $\begin{array}{c}\text { 5,4'-Dihydroxy-3,3'-dimethoxy-6:7- } \\
\text { methylenedioxyflavone } \\
{[\mathrm{M}, \mathrm{H}]}\end{array}$ & $\mathrm{C}_{18} \mathrm{H}_{14} \mathrm{O}_{8}$ & 358.0688 & $(\mathrm{M}+\mathrm{Na})^{+}$ & 381.0579 & 381.0581 & 0.39 & $\begin{array}{l}349.0312,333.4380 \\
328.4933,273.3009, \\
243.5325,189.0203\end{array}$ & DB \\
\hline 67 & 4.999 & $\begin{array}{l}\text { 1,3,5-Trihydroxy-6,7-dimethoxy-2- } \\
\text { methylantraquinone }[\mathrm{H}]\end{array}$ & $\mathrm{C}_{16} \mathrm{H}_{10} \mathrm{O}_{7}$ & 330.0734 & $(\mathrm{M}-\mathrm{H})^{-}$ & 329.0661 & 329.0667 & 1.74 & $\begin{array}{c}\text { 314.0427, 299.0207, } \\
288.0280,285.077, \\
273.0031,270.0525, \\
258.0168\end{array}$ & $\mathrm{MB}, \mathrm{TB}, \mathrm{DB}, \mathrm{DT}$ \\
\hline 68 & 5.007 & $\begin{array}{l}\text { Ventilagodenin A; } \\
\text { or 5-De-O-methyltoddanol }[\mathrm{M}, \mathrm{H}]\end{array}$ & $\mathrm{C}_{15} \mathrm{H}_{16} \mathrm{O}_{5}$ & 276.1000 & $(\mathrm{M}+\mathrm{H})^{+}$ & 277.1074 & 277.1071 & -1.21 & $\begin{array}{c}259.0957,244.0731 \\
235.0973,199.0748 \\
171.0804\end{array}$ & $\mathrm{MB}, \mathrm{TB}, \mathrm{DB}, \mathrm{DT}$ \\
\hline & & & & & $(\mathrm{M}-\mathrm{H})^{-}$ & 275.0922 & 275.0925 & 1.14 & $\begin{array}{l}259.0609,245.0447 \\
231.0661,192.6885 \\
175.0355\end{array}$ & $\mathrm{MB}, \mathrm{TB}, \mathrm{DB}$ \\
\hline 69 & 5.097 & Unidentified & $\mathrm{C}_{18} \mathrm{H}_{10} \mathrm{NO}_{4}$ & 304.0612 & $(\mathrm{M}+\mathrm{Na})^{+}$ & 327.0504 & 327.0502 & -0.46 & $\begin{array}{c}287.0555,259.0604 \\
255.0288,245.0422 \\
167.0345\end{array}$ & DB \\
\hline
\end{tabular}


Table 1. Cont.

\begin{tabular}{|c|c|c|c|c|c|c|c|c|c|c|}
\hline No. & $\begin{array}{c}t_{R} \\
(\min )\end{array}$ & Compounds & $\begin{array}{l}\text { Molecular } \\
\text { Formula }\end{array}$ & Mass & Adduct Ions & $\begin{array}{c}\text { Observed } \\
m / z\end{array}$ & $\begin{array}{c}\text { Calculated } \\
\mathrm{m} / \mathrm{z}\end{array}$ & $\begin{array}{c}\Delta \\
(\mathrm{ppm})\end{array}$ & Fragment Ions $(m / z)$ & $\begin{array}{l}\text { Found in } \\
\text { Extracts }^{\text {a }}\end{array}$ \\
\hline \multirow[t]{2}{*}{70} & 5.129 & $\begin{array}{l}\text { Rhamnalpinogenin }[\mathrm{M}, \mathrm{H}] \text {; } \\
\text { or Ventilatone B (isolation) }\end{array}$ & $\mathrm{C}_{17} \mathrm{H}_{12} \mathrm{O}_{7}$ & 328.0589 & $(\mathrm{M}+\mathrm{H})^{+}$ & 329.0659 & 329.0656 & -1.09 & $\begin{array}{l}311.0551,287.0551 \\
259.0607,167.0345 \\
312.0273,284.0326\end{array}$ & $\mathrm{MB}, \mathrm{TB}, \mathrm{DB}, \mathrm{DT}$ \\
\hline & & & & & $(\mathrm{M}-\mathrm{H})^{-}$ & 327.0508 & 327.0510 & 0.63 & $\begin{array}{c}269.0092,256.0378 \\
185.0239\end{array}$ & DB \\
\hline 71 & 5.135 & Unidentified & $\mathrm{C}_{12} \mathrm{H}_{8} \mathrm{~N}_{5} \mathrm{O}_{7} \mathrm{~S}$ & 366.0142 & $(\mathrm{M}+\mathrm{H})^{+}$ & 367.0212 & 367.0217 & 1.49 & $\begin{array}{l}352.3162,309.0637 \\
277.0991,235.8736 \\
186.9023,123.1163\end{array}$ & MB \\
\hline 72 & 5.152 & $\begin{array}{c}\text { 3,5,7-Trihydroxy-4',6-dimethoxyflavanone [M, } \\
\mathrm{H}]\end{array}$ & $\mathrm{C}_{17} \mathrm{H}_{16} \mathrm{O}_{7}$ & 332.0891 & $(\mathrm{M}+\mathrm{HCOO})^{-}$ & 377.0873 & 377.0878 & 1.30 & $\begin{array}{c}\text { 317.0660, 306.0738, } \\
259.0245,174.9557 \\
130.9658\end{array}$ & $\mathrm{MB}, \mathrm{DB}$ \\
\hline \multirow[t]{2}{*}{73} & 5.265 & Mukurozidiol (M, H) & $\mathrm{C}_{17} \mathrm{H}_{18} \mathrm{O}_{7}$ & 334.1051 & $(\mathrm{M}+\mathrm{H})^{+}$ & 335.1123 & 335.1125 & 0.77 & $\begin{array}{l}303.0866,285.0752, \\
275.0914,261.0750 \\
245.0448,233.0425\end{array}$ & $\mathrm{MB}, \mathrm{DB}, \mathrm{DT}$ \\
\hline & & & & & $(\mathrm{M}+\mathrm{HCOO})^{-}$ & 379.1026 & 379.1035 & 2.23 & $\begin{array}{c}308.0893,305.0640 \\
277.0688,262.0477 \\
174.9575\end{array}$ & $\mathrm{MB}, \mathrm{DB}$ \\
\hline 74 & 5.288 & Unidentified & $\mathrm{C}_{13} \mathrm{H}_{20} \mathrm{~N}_{3} \mathrm{O}_{8} \mathrm{~S}$ & 378.0957 & $(\mathrm{M}+\mathrm{H})^{+}$ & 379.1026 & 379.1044 & 4.68 & $\begin{array}{l}364.0528,291.0863 \\
215.0331,115.0550\end{array}$ & $\mathrm{MB}$ \\
\hline 75 & 5.296 & Unidentified & $\mathrm{C}_{19} \mathrm{H}_{22} \mathrm{O}_{10}$ & 410.1214 & $(\mathrm{M}+\mathrm{Na})^{+}$ & 433.1106 & 433.1105 & -0.13 & $\begin{array}{l}373.0897,342.0707 \\
327.0475\end{array}$ & MB \\
\hline 76 & 5.420 & Genistin $[\mathrm{M}, \mathrm{H}]$ & $\mathrm{C}_{21} \mathrm{H}_{20} \mathrm{O}_{10}$ & 432.1036 & $(\mathrm{M}+\mathrm{H})^{+}$ & 433.1109 & 433.1129 & 4.76 & $\begin{array}{c}401.0843,373.0894 \\
369.0579,342.0711 \\
327.0470\end{array}$ & DB \\
\hline \multirow[t]{2}{*}{77} & 5.463 & $6^{\prime}$-Hydroxyangolensin $[\mathrm{M}, \mathrm{H}]$ & $\mathrm{C}_{16} \mathrm{H}_{16} \mathrm{O}_{5}$ & 288.1000 & $(\mathrm{M}+\mathrm{H})^{+}$ & 289.1073 & 289.1071 & -0.92 & $\begin{array}{c}271.0967,247.0966 \\
243.1013,229.0856 \\
205.0864\end{array}$ & DB \\
\hline & & & & & $(\mathrm{M}-\mathrm{H})^{-}$ & 287.0920 & 287.0925 & 1.70 & $\begin{array}{l}269.0821,254.0605 \\
245.0823,203.0702\end{array}$ & DB \\
\hline 78 & 5.578 & (S)-Rutaretin $[\mathrm{M}, \mathrm{H}]$ & $\mathrm{C}_{14} \mathrm{H}_{14} \mathrm{O}_{5}$ & 262.0835 & $(\mathrm{M}-\mathrm{H})^{-}$ & 261.0761 & 261.0768 & 2.71 & $\begin{array}{l}246.0527,231.0291, \\
218.0561,203.0352\end{array}$ & DB \\
\hline 79 & 5.611 & Unidentified & $\mathrm{C}_{35} \mathrm{H}_{30} \mathrm{O}_{11}$ & 626.1773 & $(\mathrm{M}+\mathrm{HCOO})^{-}$ & 671.1753 & 671.177 & 2.60 & $\begin{array}{c}509.1242,416.1098, \\
254.0577 \\
243.0656,215.0705\end{array}$ & $\mathrm{~TB}, \mathrm{TD}$ \\
\hline 80 & 5.650 & Pratenol A $[\mathrm{M}, \mathrm{H}]$ & $\mathrm{C}_{14} \mathrm{H}_{12} \mathrm{O}_{5}$ & 260.0687 & $(\mathrm{M}+\mathrm{H})^{+}$ & 261.0759 & 261.0757 & -0.49 & $\begin{array}{c}200.0470,187.0749 \\
159.0439\end{array}$ & DB \\
\hline 81 & 5.743 & Gingerenone $\mathrm{C}[\mathrm{M}, \mathrm{H}]$ & $\mathrm{C}_{20} \mathrm{H}_{22} \mathrm{O}_{4}$ & 326.1521 & $(\mathrm{M}+\mathrm{H})^{+}$ & 327.1592 & 327.1591 & -0.46 & $\begin{array}{c}203.1049,171.0802, \\
151.0758,148.1110 \\
137.0600\end{array}$ & DB \\
\hline 82 & 5.848 & Unidentified & $\mathrm{C}_{53} \mathrm{H}_{26} \mathrm{~N}_{3} \mathrm{O}_{2}$ & 736.2027 & $(\mathrm{M}+\mathrm{Na})^{+}$ & 759.1920 & 759.1917 & -0.37 & $\begin{array}{l}\text { 664.0398, 504.1286, } \\
418.1196,299.0856, \\
256.0729\end{array}$ & MB \\
\hline 83 & 6.222 & Afzelechin $[\mathrm{M}, \mathrm{H}]$ & $\mathrm{C}_{15} \mathrm{H}_{14} \mathrm{O}_{5}$ & 274.0841 & $(\mathrm{M}-\mathrm{H})^{-}$ & 273.0768 & 273.0768 & 0.29 & $229.0501,202.026$ & $\mathrm{MB}, \mathrm{DB}$ \\
\hline
\end{tabular}


Table 1. Cont

\begin{tabular}{|c|c|c|c|c|c|c|c|c|c|c|}
\hline No. & $\begin{array}{c}t_{\mathrm{R}} \\
(\mathrm{min})\end{array}$ & Compounds & $\begin{array}{l}\text { Molecular } \\
\text { Formula }\end{array}$ & Mass & Adduct Ions & $\begin{array}{c}\text { Observed } \\
m / z\end{array}$ & $\begin{array}{c}\text { Calculated } \\
m / z\end{array}$ & $\begin{array}{c}\Delta \\
(\mathrm{ppm})\end{array}$ & Fragment Ions $(m / z)$ & $\begin{array}{l}\text { Found in } \\
\text { Extracts }{ }^{\mathrm{a}}\end{array}$ \\
\hline 84 & 6.342 & Ducunolide E [M, H] & $\mathrm{C}_{26} \mathrm{H}_{28} \mathrm{O}_{9}$ & 484.1724 & $(\mathrm{M}-\mathrm{H})^{-}$ & 483.1650 & 483.1661 & 2.19 & $\begin{array}{l}468.1412,439.1389 \\
424.1156,409.0887\end{array}$ & DB \\
\hline \multirow[t]{2}{*}{85} & 6.472 & Rhamnocitrin $[\mathrm{M}]$ & $\mathrm{C}_{16} \mathrm{H}_{12} \mathrm{O}_{6}$ & 300.0637 & $(\mathrm{M}+\mathrm{H})^{+}$ & 301.0711 & 301.0707 & -1.35 & $\begin{array}{l}286.0458,179.03331 \\
167.0344,121.0286 \\
284.0310,271.0605\end{array}$ & $\mathrm{MB}, \mathrm{DB}, \mathrm{DT}$ \\
\hline & & & & & $(\mathrm{M}-\mathrm{H})^{-}$ & 299.0556 & 299.0561 & 1.78 & $\begin{array}{c}240.0420,178.0257 \\
165.0189\end{array}$ & $\mathrm{MB}, \mathrm{DB}, \mathrm{DT}$ \\
\hline 86 & 6.607 & 7-Hydroxy-3,4',8-trimethoxyflavone [M, H] & $\mathrm{C}_{18} \mathrm{H}_{16} \mathrm{O}_{6}$ & 328.0949 & $(\mathrm{M}+\mathrm{H})^{+}$ & 329.1023 & 329.1020 & -1.04 & $\begin{array}{c}314.0786,313.0702, \\
285.0766,198.0922 \\
121.1025\end{array}$ & DB \\
\hline 87 & 6.698 & Acerosin $[\mathrm{M}, \mathrm{H}]$ & $\mathrm{C}_{18} \mathrm{H}_{16} \mathrm{O}_{8}$ & 360.0834 & $(\mathrm{M}-\mathrm{H})^{-}$ & 359.0761 & 359.0772 & 3.11 & $\begin{array}{l}344.0538,297.0054 \\
269.0084,171.2585\end{array}$ & $\mathrm{MB}, \mathrm{DB}$ \\
\hline 88 & 6.732 & Unidentified & $\mathrm{C}_{13} \mathrm{H}_{13} \mathrm{~N}_{6} \mathrm{O}_{7}$ & 365.0841 & $(\mathrm{M}+2 \mathrm{Na})^{+2}$ & 205.5309 & 205.5315 & 2.91 & $\begin{array}{l}\text { 320.7446, 254.9948, } \\
\text { 205.1755, 155.0088, } \\
141.5110,112.4964\end{array}$ & MB \\
\hline 89 & 6.766 & Alfalone $[\mathrm{M}, \mathrm{H}]$ & $\mathrm{C}_{17} \mathrm{H}_{14} \mathrm{O}_{5}$ & 298.0841 & $(\mathrm{M}+\mathrm{H})^{+}$ & 299.0916 & 299.0914 & -0.54 & $\begin{array}{l}271.3851,213.8909, \\
189.0528,112.7128 \\
316.0577,299.0542,\end{array}$ & DB, DT \\
\hline \multirow[t]{2}{*}{90} & 6.775 & Rhamnazin $[\mathrm{M}, \mathrm{H}]$ & $\mathrm{C}_{17} \mathrm{H}_{14} \mathrm{O}_{7}$ & 330.0743 & $(\mathrm{M}+\mathrm{H})^{+}$ & 331.0816 & 331.0812 & -1.22 & $\begin{array}{c}288,0634,179.0327 \\
167.0338\end{array}$ & $\mathrm{MB}, \mathrm{DB}$ \\
\hline & & & & & $(\mathrm{M}-\mathrm{H})^{-}$ & 329.0664 & 329.0667 & 0.74 & $\begin{array}{c}\text { 315.0457, 314.0424, } \\
286.0478,254.0217, \\
241.051,170.0353 \\
244.0734,241.0863\end{array}$ & $\mathrm{MB}, \mathrm{DB}$ \\
\hline 91 & 6.924 & Xanthoxyletin $[\mathrm{M}, \mathrm{H}]$ & $\mathrm{C}_{15} \mathrm{H}_{14} \mathrm{O}_{4}$ & 258.0894 & $(\mathrm{M}+\mathrm{H})^{+}$ & 259.0967 & 259.0965 & -0.73 & $\begin{array}{c}226.0628,217.0862, \\
213.0906,195.0799, \\
167.0879\end{array}$ & DB \\
\hline \multirow[t]{2}{*}{92} & 6.981 & Barpisoflavone A $[\mathrm{M}, \mathrm{H}]$ & $\mathrm{C}_{16} \mathrm{H}_{12} \mathrm{O}_{6}$ & 300.0636 & $(\mathrm{M}+\mathrm{H})^{+}$ & 301.0708 & 301.0707 & -0.43 & $\begin{array}{c}287.0570,269.0441, \\
236.9047,185.0603, \\
127.0056\end{array}$ & $\mathrm{MB}, \mathrm{TB}, \mathrm{DT}$ \\
\hline & & & & & $(\mathrm{M}-\mathrm{H})^{-}$ & 299.0560 & 299.0561 & 0.24 & $\begin{array}{c}267.0297,240.0422 \\
212.0476\end{array}$ & $\mathrm{MB}, \mathrm{TB}, \mathrm{DB}$ \\
\hline \multirow[t]{2}{*}{93} & 7.015 & $(+)-(R)$-Ventilagolin $[S]$ & $\mathrm{C}_{17} \mathrm{H}_{16} \mathrm{O}_{7}$ & 332.0897 & $(\mathrm{M}+\mathrm{H})^{+}$ & 333.0971 & 333.0969 & 0.60 & $\begin{array}{l}\text { 318.0736, 301.0710, } \\
\text { 276.0630, 259.0606, } \\
213.0544,185.0596\end{array}$ & $\mathrm{MB}, \mathrm{DB}, \mathrm{DT}$ \\
\hline & & & & & $(\mathrm{M}+\mathrm{HCOO})^{-}$ & 377.0873 & 377.0878 & 1.30 & $\begin{array}{c}317.066,306.0738 \\
303.0506,259.0245 \\
174.9557\end{array}$ & $\mathrm{MB}, \mathrm{DB}, \mathrm{DT}$ \\
\hline
\end{tabular}


Table 1. Cont

\begin{tabular}{|c|c|c|c|c|c|c|c|c|c|c|}
\hline No. & $\underset{(\min )}{\substack{t_{R}\\
}}$ & Compounds & $\begin{array}{l}\text { Molecular } \\
\text { Formula }\end{array}$ & Mass & Adduct Ions & $\begin{array}{c}\text { Observed } \\
m / z\end{array}$ & $\begin{array}{c}\text { Calculated } \\
\mathrm{m} / z\end{array}$ & $\begin{array}{c}\Delta \\
\text { (ppm) }\end{array}$ & Fragment Ions $(m / z)$ & $\begin{array}{l}\text { Found in } \\
\text { Extracts }\end{array}$ \\
\hline \multirow[t]{2}{*}{94} & 7.123 & Caryatin $[\mathrm{M}, \mathrm{H}]$ & $\mathrm{C}_{17} \mathrm{H}_{14} \mathrm{O}_{7}$ & 330.0741 & $(\mathrm{M}+\mathrm{H})^{+}$ & 331.0813 & 331.0812 & -0.12 & $\begin{array}{c}299.0551,276.0625 \\
259.0611,211.3641 \\
167.0181\end{array}$ & $\mathrm{MB}, \mathrm{DB}$ \\
\hline & & & & & $(\mathrm{M}-\mathrm{H})^{-}$ & 329.0660 & 329.0667 & 2.00 & $\begin{array}{c}314.0423,299.0194, \\
286.0488,271.0240 \\
165.0184\end{array}$ & MB, MT, DB, DT \\
\hline 95 & 7.349 & Kanzonol O $[\mathrm{M}, \mathrm{H}]$ & $\mathrm{C}_{22} \mathrm{H}_{22} \mathrm{O}_{6}$ & 382.1418 & $(\mathrm{M}+\mathrm{Na})^{+}$ & 405.1310 & 405.1309 & -0.29 & $\begin{array}{c}335.0526,270.0508 \\
143.0333\end{array}$ & DB \\
\hline 96 & 7.548 & Unidentified & $\mathrm{C}_{12} \mathrm{H}_{24} \mathrm{Cl}_{2} \mathrm{~N}_{2} \mathrm{O}_{8} \mathrm{~S}$ & 426.0635 & $(\mathrm{M}+\mathrm{Na})^{+}$ & 449.0527 & 449.0523 & -0.91 & $\begin{array}{c}\text { 408.2483, 388.7627, } \\
287.1038\end{array}$ & MB \\
\hline 97 & 7.887 & Unidentified & $\mathrm{C}_{34} \mathrm{H}_{30} \mathrm{~N}_{3} \mathrm{O}_{11}$ & 656.1874 & $(\mathrm{M}-\mathrm{H})^{-}$ & 655.1800 & 655.1808 & 1.18 & $557.9872,254.0580$ & $\mathrm{MB}, \mathrm{MT}$ \\
\hline 98 & 8.003 & Unidentified & $\mathrm{C}_{33} \mathrm{H}_{28} \mathrm{~N}_{3} \mathrm{O}_{11}$ & 642.1727 & $(\mathrm{M}-\mathrm{H})^{-}$ & 641.1655 & 641.1651 & -0.57 & $\begin{array}{l}509.1224,491.1100 \\
254.0579\end{array}$ & $\mathrm{MB}, \mathrm{MT}$ \\
\hline 99 & 8.120 & Unidentified & $\mathrm{C}_{16} \mathrm{H}_{11} \mathrm{NO}$ & 233.0844 & $(\mathrm{M}+\mathrm{Na})^{+}$ & 256.0734 & 256.0733 & -0.36 & $\begin{array}{c}240.0926,210.0659 \\
1821.0653,157.0646 \\
140.9164 \\
541.1141 .523 .0991\end{array}$ & MB \\
\hline 100 & 8.507 & $\begin{array}{c}\text { Dihydromorelloflavone } \\
{[\mathrm{M}, \mathrm{H}]}\end{array}$ & $\mathrm{C}_{30} \mathrm{H}_{22} \mathrm{O}_{11}$ & 558.1161 & $(\mathrm{M}+\mathrm{H})^{+}$ & 559.1236 & 559.1235 & -0.15 & $\begin{array}{c}517.1109,513.1141 \\
499.1013,313.0354 \\
257.0795\end{array}$ & DB \\
\hline \multirow[t]{2}{*}{101} & 8.938 & Emodin $[M, H, S]$ & $\mathrm{C}_{15} \mathrm{H}_{10} \mathrm{O}_{5}$ & 270.0528 & $(\mathrm{M}+\mathrm{H})^{+}$ & 271.0601 & 271.0601 & -0.16 & $\begin{array}{c}229.0509,225.0560, \\
201.0539,197.0590 \\
140.0222\end{array}$ & MB, MT, DB, DT \\
\hline & & & & & $(\mathrm{M}-\mathrm{H})^{-}$ & 269.0452 & 269.0455 & 1.33 & $\begin{array}{c}241.0511,225.0562 \\
210.0316,195.0415 \\
135.0911\end{array}$ & $\mathrm{MB}, \mathrm{MT}, \mathrm{DB}, \mathrm{DT}$ \\
\hline 102 & 9.187 & Formononetin $[\mathrm{M}, \mathrm{H}]$ & $\mathrm{C}_{16} \mathrm{H}_{12} \mathrm{O}_{4}$ & 268.0740 & $(\mathrm{M}+\mathrm{H})^{+}$ & 269.0813 & 269.0808 & -1.60 & $\begin{array}{l}254.0572,239.0708, \\
226.0618,151.0543\end{array}$ & DB \\
\hline 103 & 9.383 & $6 \alpha$-Hydroxymaackiain $[\mathrm{M}, \mathrm{H}]$ & $\mathrm{C}_{16} \mathrm{H}_{12} \mathrm{O}_{6}$ & 300.0637 & $(\mathrm{M}+\mathrm{H})^{+}$ & 301.0709 & 301.0707 & -0.76 & $255.0638,117.0696$ & $\mathrm{MB}, \mathrm{DB}$ \\
\hline 104 & 9.986 & Unidentified & $\mathrm{C}_{22} \mathrm{H}_{18} \mathrm{~N}_{7} \mathrm{O}_{3}$ & 428.1473 & $(\mathrm{M}+\mathrm{Na})^{+}$ & 451.1363 & 451.1363 & -0.01 & $\begin{array}{c}319.0570,292.0353 \\
133.0864\end{array}$ & DB \\
\hline 105 & 9.991 & Artonin $\mathrm{L}[\mathrm{M}, \mathrm{H}]$ & $\mathrm{C}_{22} \mathrm{H}_{20} \mathrm{O}_{7}$ & 396.1213 & $(\mathrm{M}+\mathrm{H})^{+}$ & 397.1283 & 397.1282 & -0.36 & $\begin{array}{l}337.1045,327.1201, \\
295.0939,287.0557\end{array}$ & DB \\
\hline 106 & 10.699 & $\operatorname{Muscomin}[\mathrm{M}, \mathrm{H}]$ & $\mathrm{C}_{18} \mathrm{H}_{18} \mathrm{O}_{7}$ & 346.1053 & $(\mathrm{M}+\mathrm{H})^{+}$ & 347.1125 & 347.1125 & -0.01 & $\begin{array}{c}332.0896,315.0864, \\
290.0781,273.0764, \\
227.0696\end{array}$ & DB \\
\hline 107 & 10.824 & Unidentified & $\mathrm{C}_{15} \mathrm{H}_{11} \mathrm{O}_{4}$ & 255.0658 & $(\mathrm{M}+\mathrm{H})^{+}$ & 256.0731 & 256.073 & -0.41 & $\begin{array}{c}241.0502,238.0625 \\
210.0683,198.9302 \\
182.0727\end{array}$ & $\mathrm{MB}, \mathrm{MT}, \mathrm{DB}$ \\
\hline
\end{tabular}


Table 1. Cont

\begin{tabular}{|c|c|c|c|c|c|c|c|c|c|c|}
\hline No. & $\begin{array}{c}t_{\mathrm{R}} \\
(\mathrm{min})\end{array}$ & Compounds & $\begin{array}{l}\text { Molecular } \\
\text { Formula }\end{array}$ & Mass & Adduct Ions & $\begin{array}{c}\text { Observed } \\
m / z\end{array}$ & $\begin{array}{c}\text { Calculated } \\
\mathrm{m} / \mathrm{z}\end{array}$ & $\begin{array}{c}\Delta \\
(\mathrm{ppm})\end{array}$ & Fragment Ions $(m / z)$ & $\begin{array}{l}\text { Found in } \\
\text { Extracts a }\end{array}$ \\
\hline 108 & 11.423 & $\begin{array}{c}2^{\prime}, 3,5 \text {-Trihydroxy-5',7-dimethoxyflavanone } \\
{[\mathrm{M}, \mathrm{H}]}\end{array}$ & $\mathrm{C}_{19} \mathrm{H}_{20} \mathrm{O}_{9}$ & 332.0885 & $\left(\mathrm{M}+\mathrm{CH}_{3} \mathrm{COO}\right)^{-}$ & 391.1024 & 391.1035 & 2.64 & $\begin{array}{c}317.0658,302.0387 \\
242.6421,209.8790 \\
130.2329\end{array}$ & DB \\
\hline 109 & 11.796 & Palmidin A [M, H] & $\mathrm{C}_{30} \mathrm{H}_{22} \mathrm{O}_{8}$ & 510.1312 & $\begin{array}{l}(\mathrm{M}+\mathrm{H})^{+} \\
(\mathrm{M}-\mathrm{H})^{-}\end{array}$ & $\begin{array}{l}511.1387 \\
509.1238\end{array}$ & $\begin{array}{l}511.1387 \\
509.1242\end{array}$ & $\begin{array}{l}0.16 \\
0.80\end{array}$ & $\begin{array}{c}256.0733,133.0854 \\
254.0583\end{array}$ & $\begin{array}{l}\text { MB, MT, DB, DT } \\
\text { MB, MT, DB, DT }\end{array}$ \\
\hline \multirow[t]{2}{*}{110} & 12.237 & $\begin{array}{l}\text { 1,3,5,8-Tetrahydroxy-6-methoxy-2- } \\
\text { methylanthraquinone }[\mathrm{M}, \mathrm{H}]\end{array}$ & $\mathrm{C}_{16} \mathrm{H}_{12} \mathrm{O}_{7}$ & 316.0585 & $(\mathrm{M}+\mathrm{H})^{+}$ & 317.0658 & 317.0656 & -0.56 & $\begin{array}{c}299.0575,254.8649 \\
193.0125,135.1168 \\
127.0534\end{array}$ & MB, DB \\
\hline & & & & & $(\mathrm{M}-\mathrm{H})^{-}$ & 315.0505 & 315.0510 & 1.57 & $\begin{array}{l}\text { 300.0261, 272.0305, } \\
\text { 216.9344, } 163.1615 \\
112.9849\end{array}$ & MB \\
\hline 111 & 12.742 & Khelmarin D [M, H] & $\mathrm{C}_{28} \mathrm{H}_{24} \mathrm{O}_{8}$ & 488.1460 & $\left(\mathrm{M}+\mathrm{CH}_{3} \mathrm{COO}\right)^{-}$ & 547.1599 & 547.1610 & 2.00 & $\begin{array}{c}457.0900 \\
469.0870,400.8285\end{array}$ & DB \\
\hline 112 & 12.798 & Amentoflavone $[\mathrm{M}, \mathrm{H}]$ & $\mathrm{C}_{30} \mathrm{H}_{18} \mathrm{O}_{10}$ & 538.0889 & $(\mathrm{M}-\mathrm{H})^{-}$ & 537.0814 & 537.0827 & 2.44 & $\begin{array}{c}333.5261,173.9422 \\
107.5508\end{array}$ & $\mathrm{MB}, \mathrm{MT}, \mathrm{DB}$ \\
\hline 113 & 12.837 & Isophysalin $\mathrm{G}[\mathrm{M}, \mathrm{H}]$ & $\mathrm{C}_{28} \mathrm{H}_{30} \mathrm{O}_{10}$ & 526.1860 & $(\mathrm{M}+\mathrm{Na})^{+}$ & 549.1752 & 549.1731 & -3.87 & $\begin{array}{c}\text { 517.1481, } 475.1364 \\
246.0893\end{array}$ & DB \\
\hline 114 & 13.326 & Yuccaol C [M, H] & $\mathrm{C}_{30} \mathrm{H}_{22} \mathrm{O}_{10}$ & 542.1201 & $(\mathrm{M}-\mathrm{H})^{-}$ & 541.1126 & 541.1140 & 2.6 & $\begin{array}{c}523.0998,511.0683 \\
493.0539,308.0347 \\
231.1206\end{array}$ & DB \\
\hline 115 & 13.632 & Ephedrannin A [M, H] & $\mathrm{C}_{30} \mathrm{H}_{20} \mathrm{O}_{11}$ & 556.0997 & $\left(\mathrm{M}+\mathrm{CH}_{3} \mathrm{COO}\right)^{-}$ & 615.1134 & 615.1144 & 1.59 & $299.0208,289.0709$ & DB \\
\hline 116 & 14.355 & Unidentified & $\mathrm{C}_{29} \mathrm{H}_{22} \mathrm{~N}_{3} \mathrm{O}_{7}$ & 524.1462 & $(\mathrm{M}-\mathrm{H})^{-}$ & 523.1387 & 523.1385 & -0.47 & 254.0580 & $\mathrm{MB}$ \\
\hline 117 & 14.718 & Unidentified & $\mathrm{C}_{16} \mathrm{H}_{13} \mathrm{O}_{4}$ & 269.0814 & $(\mathrm{M}+\mathrm{H})^{+}$ & 270.0885 & 270.0887 & 0.78 & $\begin{array}{c}\text { 227.07006, 179.0025, } \\
151.9915,105.0345 \\
386.1758,340.4709\end{array}$ & MB \\
\hline 118 & 14.748 & Palmidin B [M, H] & $\mathrm{C}_{30} \mathrm{H}_{22} \mathrm{O}_{7}$ & 494.1349 & $(\mathrm{M}-\mathrm{H})^{-}$ & 493.1279 & 493.1293 & 2.74 & $\begin{array}{l}254.0581,224.0460 \\
213.0023,161.4482\end{array}$ & DB, DT \\
\hline 119 & 16.060 & Murrayazolinine $[\mathrm{M}, \mathrm{H}]$ & $\mathrm{C}_{23} \mathrm{H}_{27} \mathrm{NO}_{2}$ & 349.2042 & $\left(\mathrm{M}+\mathrm{NH}_{4}\right)^{+}$ & 367.2390 & 367.2380 & -2.81 & $\begin{array}{c}323.2308,268.2613 \\
172.1157,156.1387 \\
116.0538\end{array}$ & MB, MT, DB. DT \\
\hline 120 & 16.060 & Unidentified & $\mathrm{C}_{32} \mathrm{H}_{28} \mathrm{~N}_{2} \mathrm{~S}_{3}$ & 536.1416 & $(\mathrm{M}+\mathrm{Na})^{+}$ & 559.1318 & 559.1307 & -1.94 & $\begin{array}{l}521.0807,466.7954, \\
409.8348,401.2433\end{array}$ & MB \\
\hline 121 & 17.810 & Rheidin B [M, H] & $\mathrm{C}_{30} \mathrm{H}_{20} \mathrm{O}_{8}$ & 508.1146 & $(\mathrm{M}-\mathrm{H})^{-}$ & 507.1074 & 507.1085 & 2.24 & $\begin{array}{l}479.1105,304.9145 \\
259.2411,149.1327\end{array}$ & $\mathrm{MB}, \mathrm{MT}, \mathrm{DT}$ \\
\hline 122 & 18.371 & Copalic acid $[\mathrm{M}, \mathrm{H}]$ & $\mathrm{C}_{20} \mathrm{H}_{32} \mathrm{O}_{2}$ & 304.2407 & $(\mathrm{M}+\mathrm{H})^{+}$ & 305.2479 & 305.2475 & -1.40 & $\begin{array}{c}137.1326,123.1165 \\
109.1010 \\
231.2105,175.1484\end{array}$ & $\mathrm{MB}$ \\
\hline 123 & 20.707 & $\gamma$-Pinacene $[\mathrm{M}, \mathrm{H}]$ & $\mathrm{C}_{20} \mathrm{H}_{32}$ & 272.2506 & $(\mathrm{M}+\mathrm{H})^{+}$ & 273.2578 & 273.2577 & -0.45 & $\begin{array}{l}\text { 163.1482, 149.1327, } \\
\text { 135.1169, 121.1014, } \\
\text { 109.1013, 107.0856 }\end{array}$ & DB \\
\hline 124 & 20.750 & Pipericine $[\mathrm{M}, \mathrm{H}]$ & $\mathrm{C}_{22} \mathrm{H}_{41} \mathrm{NO}$ & 335.3190 & $(\mathrm{M}+\mathrm{H})^{+}$ & 336.3264 & 336.3261 & -0.90 & $\begin{array}{c}240.2341,184.1702 \\
142.1230,170.1534 \\
100.0761\end{array}$ & MB, MT, DB, DT \\
\hline
\end{tabular}


Table 1. Cont.

\begin{tabular}{|c|c|c|c|c|c|c|c|c|c|c|}
\hline No. & $\begin{array}{c}t_{R} \\
(\min )\end{array}$ & Compounds & $\begin{array}{l}\text { Molecular } \\
\text { Formula }\end{array}$ & Mass & Adduct Ions & $\begin{array}{c}\text { Observed } \\
\mathrm{m} / \mathrm{z}\end{array}$ & $\begin{array}{c}\text { Calculated } \\
\mathrm{m} / \mathrm{z}\end{array}$ & $\begin{array}{c}\Delta \\
(\mathrm{ppm})\end{array}$ & Fragment Ions $(m / z)$ & $\begin{array}{l}\text { Found in } \\
\text { Extracts }\end{array}$ \\
\hline \multirow[t]{2}{*}{125} & 22.976 & Araliacerebroside $[\mathrm{M}, \mathrm{H}]$ & $\mathrm{C}_{40} \mathrm{H}_{77} \mathrm{NO}_{10}$ & 731.5543 & $(\mathrm{M}+\mathrm{Na})^{+}$ & 754.5435 & 754.5440 & 0.63 & ND & $\mathrm{MB}, \mathrm{MT}, \mathrm{DT}$ \\
\hline & & & & & $(\mathrm{M}-\mathrm{H})^{-}$ & 730.5462 & 730.5475 & 1.71 & $\begin{array}{c}568.4923,416.3272 \\
326.2700,271.2258 \\
179.0551,131.0328 \\
119.0354 \\
476.3663,371.2275\end{array}$ & MB, MT \\
\hline 126 & 23.282 & Unidentified & $\mathrm{C}_{26} \mathrm{H}_{51} \mathrm{~N}_{13}$ & 545.4386 & $(\mathrm{M}+\mathrm{Na})^{+}$ & 568.4274 & 568.4283 & 1.52 & $\begin{array}{c}250.1754,185.1303 \\
133.0845\end{array}$ & MB \\
\hline 127 & 24.262 & Unidentified & $\mathrm{C}_{26} \mathrm{H}_{45} \mathrm{~N}_{4}$ & 413.3639 & $(\mathrm{M}+\mathrm{H})^{+}$ & 414.3710 & 414.3717 & 1.59 & 112.0989 & MB \\
\hline 128 & 24.301 & Unidentified & $\mathrm{C}_{26} \mathrm{H}_{49} \mathrm{NO}$ & 391.3819 & $(\mathrm{M}+\mathrm{H})^{+}$ & 392.3893 & 392.3887 & -1.51 & $\begin{array}{l}282.2781,198.1852, \\
156.1385,130.1590\end{array}$ & $\mathrm{MB}, \mathrm{MT}$ \\
\hline 129 & 24.466 & Unidentified & $\mathrm{C}_{36} \mathrm{H}_{38} \mathrm{~N}_{4} \mathrm{O}_{5}$ & 606.2843 & $(\mathrm{M}+\mathrm{H})^{+}$ & 607.2917 & 607.2915 & -0.35 & 547.27 & MB \\
\hline 130 & 24.500 & Clerosterol 3-glucoside $[\mathrm{M}, \mathrm{H}]$ & $\mathrm{C}_{35} \mathrm{H}_{58} \mathrm{O}_{6}$ & 574.4219 & $\left(\mathrm{M}+\mathrm{CH}_{3} \mathrm{COO}\right)^{-}$ & 633.4359 & 633.4372 & 2.00 & $\begin{array}{c}559.3987,541.3890 \\
383.3517,175.0401 \\
133.0300\end{array}$ & DB \\
\hline 131 & 24.755 & Unidentified & $\mathrm{C}_{24} \mathrm{H}_{25} \mathrm{~N}_{9} \mathrm{O}_{2} \mathrm{~S}_{2}$ & 535.1579 & $(\mathrm{M}+\mathrm{H})^{+}$ & 536.1658 & 536.1645 & -2.30 & $\begin{array}{c}\text { 503.1070, } 415.0364 \\
\text { 341.0176, } 221.0841 \\
147.0655\end{array}$ & $\mathrm{MB}, \mathrm{TB}$ \\
\hline 132 & 24.913 & Unidentified & $\mathrm{C}_{37} \mathrm{H}_{38} \mathrm{~N}_{5} \mathrm{O}_{2}$ & 584.3020 & $(\mathrm{M}+\mathrm{Na})^{+}$ & 607.2911 & 607.2918 & 1.17 & $\begin{array}{l}547.2713,460.2258, \\
367.0213,280.2360 \\
167.1421,107.0840\end{array}$ & MB \\
\hline \multirow[t]{2}{*}{133} & 25.117 & AS $1-5[M, H]$ & $\mathrm{C}_{40} \mathrm{H}_{77} \mathrm{NO}_{9}$ & 715.5597 & $(\mathrm{M}+\mathrm{Na})^{+}$ & 738.5489 & 738.5489 & 0.15 & ND & MB, MT, DT \\
\hline & & & & & $(\mathrm{M}+\mathrm{HCOO})^{-}$ & 760.5560 & 760.5580 & 2.69 & $\begin{array}{l}\text { 655.7664, 552.4965, } \\
534.4872,299.4631, \\
179.0584,101.0237 \\
385.1727,329.1716,\end{array}$ & MB, MT \\
\hline 134 & 25.474 & 3-Dehydroteasterone $[\mathrm{M}, \mathrm{H}]$ & $\mathrm{C}_{28} \mathrm{H}_{46} \mathrm{O}_{4}$ & 446.3401 & $(\mathrm{M}+\mathrm{Na})^{+}$ & 469.3293 & 469.3288 & -0.93 & $\begin{array}{c}189.0170,171.0054 \\
113.1314 \\
775.5344,613.0880\end{array}$ & MB, MT, DB, DT \\
\hline 135 & 25.552 & Unidentified & $\mathrm{C}_{42} \mathrm{H}_{74} \mathrm{~N}_{6} \mathrm{O}_{10}$ & 822.5471 & $(\mathrm{M}-\mathrm{H})^{+}$ & 821.5396 & 821.5394 & -0.32 & $\begin{array}{l}523.3704,339.4486, \\
277.2172,261.1697, \\
175.6021,103.9958\end{array}$ & MB \\
\hline 136 & 25.644 & Unidentified & $\mathrm{C}_{29} \mathrm{H}_{41} \mathrm{~N}_{2} \mathrm{O}_{2} \mathrm{~S}_{5}$ & 609.1765 & $(\mathrm{M}+\mathrm{H})^{+}$ & 610.1843 & 610.1844 & 0.30 & $\begin{array}{c}489.0548,355.0700 \\
281.0509,221.0844 \\
147.0659\end{array}$ & MB \\
\hline 137 & 26.561 & Secasterone $[\mathrm{M}, \mathrm{H}]$ & $\mathrm{C}_{28} \mathrm{H}_{46} \mathrm{O}_{4}$ & 446.3395 & $(\mathrm{M}+\mathrm{Na})^{+}$ & 469.3286 & 469.3286 & 0.44 & $\begin{array}{l}329.1732,284.1760 \\
268.0679,109.1008 \\
778.5514,713.2510\end{array}$ & $\mathrm{MB}, \mathrm{MT}$ \\
\hline 138 & 26.727 & Unidentified & $\mathrm{C}_{42} \mathrm{H}_{76} \mathrm{~N}_{6} \mathrm{O}_{10}$ & 824.5628 & $(\mathrm{M}-\mathrm{H})^{-}$ & 823.5555 & 823.5550 & -0.60 & $\begin{array}{l}\text { 657.5735, 579.3840, } \\
513.3079,456.2245, \\
388.2563,277.2178\end{array}$ & MB \\
\hline 139 & 26.799 & Unidentified & $\mathrm{C}_{36} \mathrm{H}_{76} \mathrm{~N}_{9} \mathrm{O}_{7} \mathrm{~S}$ & 778.5595 & $(\mathrm{M}+\mathrm{Na})^{+}$ & 801.5482 & 801.5482 & -0.14 & $519.2919,121.1020$ & MB \\
\hline 140 & 27.173 & Unidentified & $\mathrm{C}_{37} \mathrm{H}_{67} \mathrm{~N}_{13} \mathrm{O}_{3}$ & 741.5491 & $(\mathrm{M}+\mathrm{Na})^{+}$ & 764.5381 & 764.5382 & 0.15 & 102.0913 & MB, MT \\
\hline
\end{tabular}


Table 1. Cont.

\begin{tabular}{|c|c|c|c|c|c|c|c|c|c|c|}
\hline No. & $\begin{array}{c}t_{R} \\
(\min )\end{array}$ & Compounds & $\begin{array}{l}\text { Molecular } \\
\text { Formula }\end{array}$ & Mass & Adduct Ions & $\begin{array}{c}\text { Observed } \\
m / z\end{array}$ & $\begin{array}{c}\text { Calculated } \\
\mathrm{m} / \mathrm{z}\end{array}$ & $\begin{array}{c}\Delta \\
\text { (ppm) }\end{array}$ & Fragment Ions $(m / z)$ & $\begin{array}{l}\text { Found in } \\
\text { Extracts a }\end{array}$ \\
\hline 141 & 29.246 & Unidentified & $\mathrm{C}_{22} \mathrm{H}_{48} \mathrm{Cl}_{2} \mathrm{~N}_{5} \mathrm{O}_{2} \mathrm{~S}$ & 516.2900 & $(\mathrm{M}+\mathrm{H})^{+}$ & 517.2957 & 517.2979 & 4.07 & $\begin{array}{c}312.0957,244.0374 \\
175.9745\end{array}$ & MB, MT \\
\hline 142 & 29.348 & Unidentified & $\mathrm{C}_{34} \mathrm{H}_{68}$ & 476.5322 & $\left(\mathrm{M}+\mathrm{NH}_{4}\right)^{+}$ & 494.5662 & 494.5659 & -0.53 & $\begin{array}{c}453.3644,271.3170 \\
151.1298 \\
478.6391,447.7013\end{array}$ & MB, MT \\
\hline 143 & 30.655 & Lansiol $[\mathrm{M}, \mathrm{H}]$ & $\mathrm{C}_{33} \mathrm{H}_{56} \mathrm{O}$ & 468.4326 & $\left(\mathrm{M}+\mathrm{CH}_{3} \mathrm{COO}\right)^{-}$ & 527.4465 & 527.4470 & 0.91 & $\begin{array}{c}413.8984,365.2430 \\
305.1114,258.1590 \\
192.0016\end{array}$ & $\mathrm{MB}$ \\
\hline 144 & 32.152 & Unidentified & $\mathrm{C}_{6} \mathrm{H}_{12} \mathrm{~N}_{6} \mathrm{O}_{3}$ & 216.0977 & $(\mathrm{M}+\mathrm{H})^{+}$ & 217.1049 & 217.1044 & -2.52 & $\begin{array}{l}\text { 204.0959, 161.0979, } \\
134.0842,107.0513\end{array}$ & MB \\
\hline
\end{tabular}

a MB: MeOH crude extract of bark; MT: $\mathrm{MeOH}$ crude extract of trunk; DB: $\mathrm{CH}_{2} \mathrm{Cl}_{2}$ crude extract of bark; DT: $\mathrm{CH}_{2} \mathrm{Cl}_{2}$ crude extract of trunk. 


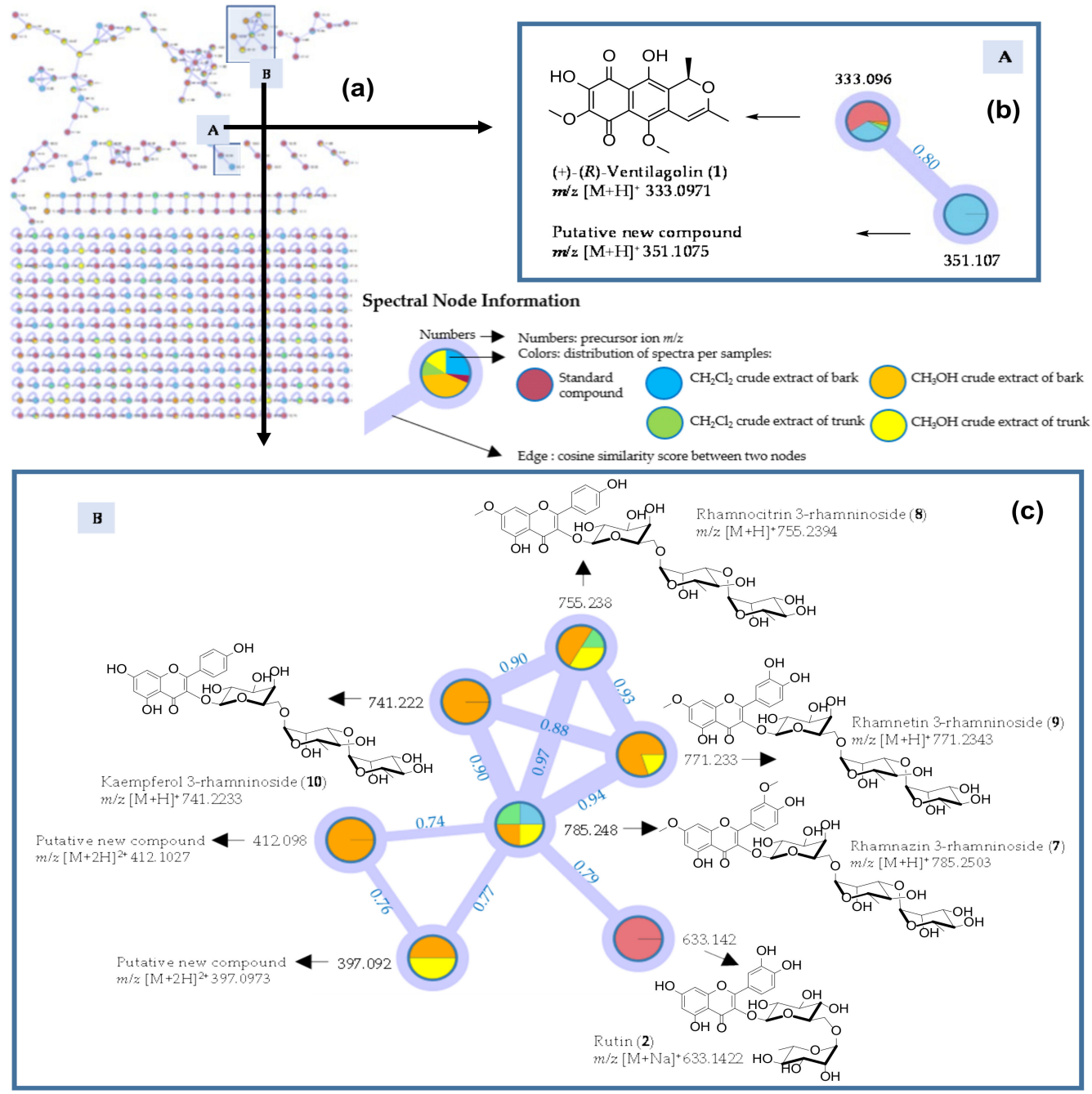

Figure 1. Molecular networking of crude extracts of $V$. denticulata as a complementary method for the dereplication strategy: (a) Molecular networking of crude extracts in a positive ionization mode; (b) Molecular networking connected to (+)- $(R)$-ventilagolin (1) and a putative new naphthalene derivative found in $\mathrm{CH}_{2} \mathrm{Cl}_{2}$ crude extract of bark; (c) Molecular networking connected to rutin (2) and other flavonol glycosides found in $\mathrm{MeOH}$ crude extract of bark.

We employed a molecular networking for the investigation of a profile of chemical constituents in crude extracts of $V$. denticulata, basically with that of crude extracts in a positive ionization mode (Figure 1a). Colors for $\mathrm{MeOH}$ extracts of bark and trunk, as well as three crude extracts of bark and trunk, are depicted in Figure 1. In the present work, $(+)-(R)$-ventilagolin (1), a naphthalene derivative, was used as a standard compound (purple color, Figure $1 \mathrm{~b}$ ) and it found in $\mathrm{MeOH}$ and $\mathrm{CH}_{2} \mathrm{Cl}_{2}$ crude extracts of bark and $\mathrm{CH}_{2} \mathrm{Cl}_{2}$ crude extract of trunk but not in $\mathrm{MeOH}$ crude extract of trunk (Table 1). The molecular networking of $(+)-(R)$-ventilagolin $(\mathbf{1})$ is in a cluster A (Figure 1b). Rutin (2), a flavonol glycoside, was also used as a standard compound and its molecular networking is in a cluster B, as shown in Figure 1c. The dereplication by MS/MS based molecular networking in a positive ionization mode also suggested the presence of a potential new naphthalene derivative (Figure 1b) and flavonol glycoside derivatives (Figure 1c), by inspecting nodes in the clusters connected to $(+)-(R)$-ventilagolin $(\mathbf{1})$ and rutin $(\mathbf{2})$, respectively.

The molecular networking of $(+)-(R)$-ventilagolin (1) $\left(\mathrm{m} / \mathrm{z} 333.0971[\mathrm{M}+\mathrm{H}]^{+}\right)($cluster A; Figure 1b) showed the node of MS/MS spectra related to the ion at $m / z 351.1075[\mathrm{M}+\mathrm{H}]^{+}$with cosine similarity score of 0.80. A putative unknown compound observed at $\mathrm{m} / \mathrm{z} 351.1075[\mathrm{M}+\mathrm{H}]^{+}$had a mass difference of 18 from (+)-(R)-ventilagolin (1) $\left(\mathrm{m} / \mathrm{z} 333.0971[\mathrm{M}+\mathrm{H}]^{+}\right.$, calcd for $\left[\mathrm{C}_{17} \mathrm{H}_{16} \mathrm{O}_{7}+\mathrm{H}\right]^{+}$, 
333.0974, $\left.\Delta_{m / z}=0.90 \mathrm{ppm}\right)$, suggesting that a putative new compound has an additional hydroxyl group. The tentative new derivative had the observed ion at $\mathrm{m} / z 351.1075[\mathrm{M}+\mathrm{H}]^{+}$, calcd for $\left[\mathrm{C}_{17} \mathrm{H}_{18} \mathrm{O}_{8}+\mathrm{H}\right]^{+}, 351.1080, \Delta_{m / z}=1.42 \mathrm{ppm}$ and thus having the molecular formula of $\mathrm{C}_{17} \mathrm{H}_{18} \mathrm{O}_{8}$. MS/MS spectra of both (+)-(R)-ventilagolin (1) and a putative new derivative showed the ions at $m / z 276$ and 259 (Figures S3 and S4, Supplementary Materials); a typical MS/MS fragmentation of $(+)-(R)$-ventilagolin (1) is depicted in Figure 2, showing the ion at $m / z 276.0630$ of $\left[\mathrm{C}_{14} \mathrm{H}_{12} \mathrm{O}_{6}\right]^{+}$, $276.0628, \Delta_{m / z}=0.72 \mathrm{ppm}$. Based upon the typical MS fragmentation of $(+)-(R)$-ventilagolin (1), the tentative structure of a new derivative observed at $\mathrm{m} / \mathrm{z} 351.1075[\mathrm{M}+\mathrm{H}]^{+}$(cluster $\mathrm{A}$; Figure $1 \mathrm{~b}$ ) is proposed to be either 3-hydroxy-ventilagolin (3) or 4-hydroxy-ventilagolin (4), as shown in Figure 2. MS/MS spectrum (Figure S4, Supplementary Materials) of a putative new compound showed that it underwent neutral loss of water, giving a fragment ion at emphm/z $333.0949[\mathrm{M}+\mathrm{H}]^{+}$, calcd for $\left[\mathrm{C}_{17} \mathrm{H}_{16} \mathrm{O}_{7}+\mathrm{H}\right]^{+}, 333.0974, \Delta_{m / z}=7.50 \mathrm{ppm}$ (Figure 2), which is of $(+)-(R)$-ventilagolin (1), which in turn, fragmented to the ion at $m / z 276.0630$, calcd for $\left[\mathrm{C}_{14} \mathrm{H}_{12} \mathrm{O}_{6}\right]^{+}$, 276.0628, $\Delta_{m / z}=0.72 \mathrm{ppm}$ (Figure 2 and Figure 44 ) that is a typical MS fragmentation for this compound class. Unfortunately, we could not isolate the putative new derivative for detailed NMR analysis. It is worth mentioning that 3-hydroxy-ventilagolin (3) has a similar structural feature to a fungal pigment, fusarubin (5) (Figure 2) [26,27], which also has an anhydro derivative, anhydrofusarubin (6) [27] (Figure 2), whose structure is similar to that of (+)-(R)-ventilagolin (1). By analogy to the structures of fusarubin (5) and anhydrofusarubin (6), the putative new compound is possibly 3-hydroxy-ventilagolin (3) (Figure 2).
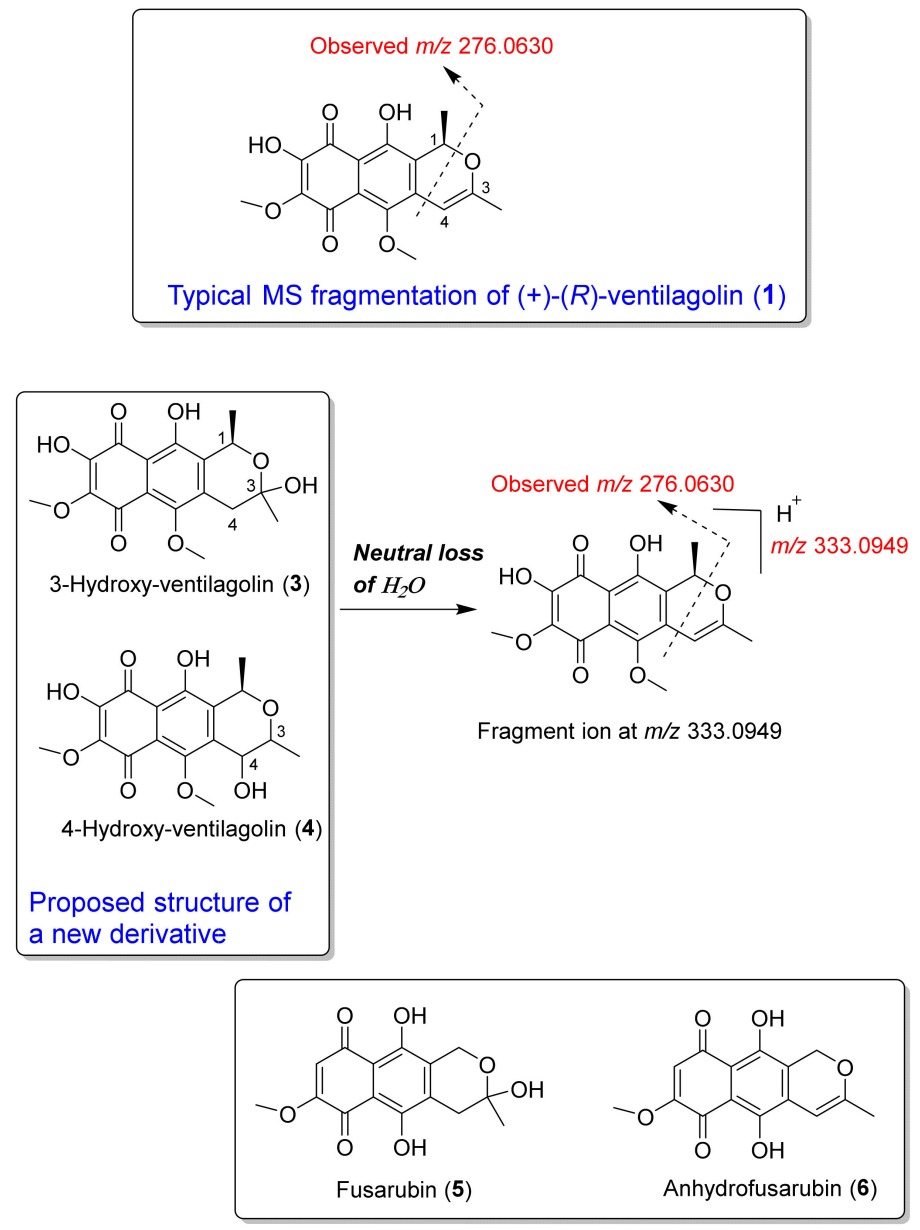

Figure 2. Typical MS fragmentation of (+)-(R)-ventilagolin (1); possible structure and MS fragmentations of a new derivative, 3-hydroxy-ventilagolin (3) or 4-hydroxy-ventilagolin (4); and structures of fusarubin (5) and anhydrofusarubin (6). 
In a cluster B (Figure 1c), node of MS/MS spectra connected to rutin (2) $(\mathrm{m} / \mathrm{z} 633.1422$ $\left.[\mathrm{M}+\mathrm{Na}]^{+}\right)$, a standard compound, possessed a precursor ion of xanthorhamnin $\mathrm{C}$ or rhamnazin 3-rhamninoside (7) (Figures $1 \mathrm{c}$ and 3) at $\mathrm{m} / \mathrm{z} 785.2503[\mathrm{M}+\mathrm{H}]^{+}$with a cosine similarity score of 0.79. Rhamnazin 3-rhamninoside (7) was isolated and characterized by analysis of $1 \mathrm{D}$ and 2D NMR spectroscopy $\left({ }^{1} \mathrm{H},{ }^{13} \mathrm{C}\right.$ NMR and MS spectra are in Figures S10-S12, Supplementary Materials). Spectroscopic data of rhamnazin 3-rhamninoside (7) were in good agreement with those reported in the literature [28]. Rhamnazin 3-rhamninoside (7) had related precursor ions at $m / z 755.2394[\mathrm{M}+\mathrm{H}]^{+}$ with a cosine similarity score of 0.97 , at $m / z 771.2343[\mathrm{M}+\mathrm{H}]^{+}$with a cosine similarity score of 0.94 and at $m / z 741.2233[\mathrm{M}+\mathrm{H}]^{+}$with a cosine similarity score of 0.90 , which are catharticin or rhamnocitrin 3-rhamninoside (8), xanthorhamnin B or rhamnetin 3-rhamninoside (9) and kaempferol 3-rhamninoside (10), respectively (Figures 1c and 3). Flavonol glycosides 8-10 were also isolated and structurally characterized by analysis of $1 \mathrm{D}$ and $2 \mathrm{D}$ NMR spectroscopy $\left({ }^{1} \mathrm{H},{ }^{13} \mathrm{C}\right.$ NMR and MS spectra are in Figures S13-S21, Supplementary Materials). Spectroscopic data of compounds 8-10 were identical to those published in the literature [28-31]. Moreover, flavovilloside or quercetin 3-rhamninoside (11) (Figure 3) was also obtained during the isolation of flavonol glycosides 7-10; its ${ }^{1} \mathrm{H},{ }^{13} \mathrm{C}$ NMR and MS spectra are in Figures S22-S24, Supplementary Materials). Spectroscopic data of quercetin 3-rhamninoside (11) were in good agreement with published values [28]. However, quercetin 3-rhamninoside (11) was not detected by LC-MS/MS analysis; therefore, it is not listed in Table 1 and it does not appear in the molecular networking of a cluster B (Figure 1c) in spite of being a derivative of rutin (2). The sugar in a standard flavonol glycoside, rutin (2), is glucose, while that in the isolated flavonol glycosides $\mathbf{7 - 1 1}$ is galactose (Figure 3). In a cluster B (Figure 1c), compounds with the ions at $m / z 412.1027[\mathrm{M}+2 \mathrm{H}]^{2+}$ and $397.0973[\mathrm{M}+2 \mathrm{H}]^{2+}$ had related precursor ions to rhamnazin 3-rhamninoside (7) and they were considered as potential new compounds. Unfortunately, attempts to isolate these compounds for detailed NMR analysis have met with failure. It is worth mentioning that HPLC-PDA method could be used to distinguish $3^{\prime}, 4^{\prime}$-dihydroxy flavonoid (i.e., flavonol glycosides 7, 9 and 11) from $4^{\prime}$-dihydroxy flavonoid derivative (i.e., flavonol glycosides 8 and 10) (Figure 3). $3^{\prime}, 4^{\prime}$-Dihydroxy flavonoid had a typical $\lambda_{\max }$ at $356 \mathrm{~nm}$ in the UV spectrum, while $4^{\prime}$-dihydroxy flavonoid derivative showed a typical $\lambda_{\max }$ at $348 \mathrm{~nm}$ (Figure 3).

Analysis of MS/MS spectrum (Figure S5, Supplementary Materials) of a standard flavonol glycoside, rutin (2), revealed losses of glucose and rhamnose, showing the ions resulting from the loss of rhamnose (at $m / z 465$ from loss of 146) and of glucose-rhamnose (at $m / z$ 303) (Figure 4). Loss of 146 of rhamnose gave the ion at $m / z 465$ and such loss was previously observed for flavonoid glycosides [25] and triterpene saponins [32]. Interestingly, the ion abundance at $m / z 147.0653$, calcd $\left[\mathrm{C}_{6} \mathrm{H}_{11} \mathrm{O}_{4}+\mathrm{H}\right]^{+}$, which was of a rhamnose fragment, was 4 times lower than that of the ion at $\mathrm{m} / \mathrm{z} 129.0547$ (Figure S5, Supplementary Materials). Careful analysis revealed that the observed ion at $m / z 129.0547$ could be of an oxonium ion of a sugar rhamnose, which was from a neutral loss of water of a rhamnose fragment at $m / z$ 147.0653, as depicted in Figure 4. The observed ion at $m / z 129.0547$ and the calculated $\mathrm{m} / \mathrm{z}$ value of 129.0546 for $\mathrm{C}_{6} \mathrm{H}_{9} \mathrm{O}_{3}{ }^{+}$with the mass difference of $0.26 \mathrm{ppm}$ (Figure 4 ) readily confirmed the structure of an oxonium ion of rhamnose. Normally, oxonium ions of sugar are observed in MS/MS spectra of glycosides [33] and they are useful ions for sugar identification in modern glycoproteomic research $[34,35]$. To the best of our knowledge, this is the first report on the oxonium ion of rhamnose, $\mathrm{C}_{6} \mathrm{H}_{9} \mathrm{O}_{3}{ }^{+}$at $\mathrm{m} / \mathrm{z} \mathrm{ca} 129.05$ and it is possibly used as a characteristic fragment ion for rhamnose in mass spectrometry. 

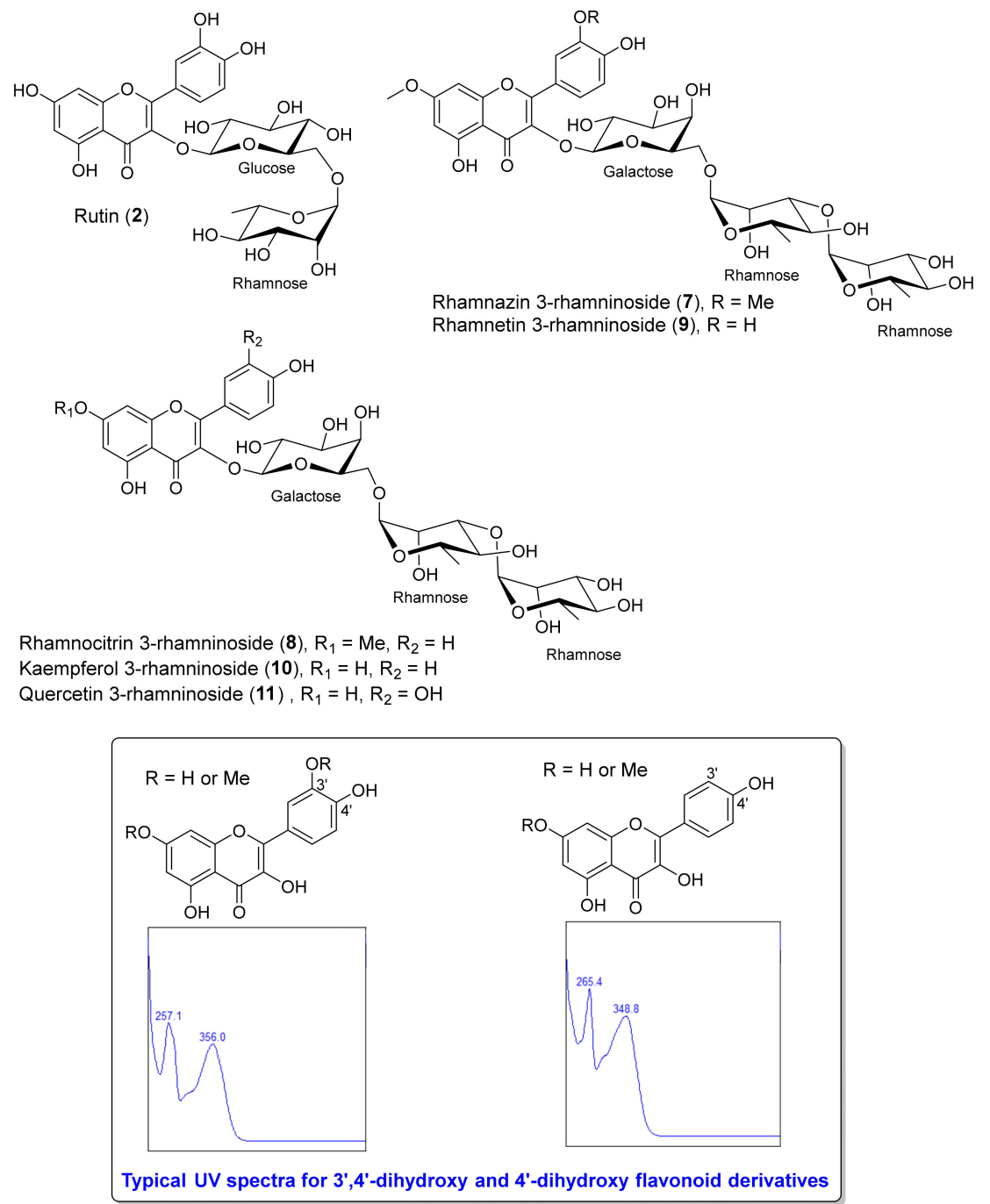

Figure 3. Structures of a standard flavonol glycoside, rutin (2) and flavonol glycosides 7-11; and typical UV spectra for $3^{\prime}, 4^{\prime}$-dihydroxy and $4^{\prime}$-dihydroxy flavonoid derivatives showing $\lambda_{\max }$ at $356 \mathrm{~nm}$ and $348 \mathrm{~nm}$, respectively.

Molecular networking of rutin (2) (cluster B, Figure 1c) had the precursor ion of xanthorhamnin $\mathrm{C}$ or rhamnazin 3-rhamninoside (7) at $\mathrm{m} / \mathrm{z} 785.2503[\mathrm{M}+\mathrm{H}]^{+}$with a cosine similarity score of 0.79. MS/MS spectrum (Figure S6, Supplementary Materials) of rhamnazin 3-rhamninoside (7) showed fragment ions analogous to that of rutin (2), that is, loss of rhamnose giving the oxonium ion at $\mathrm{m} / \mathrm{z} 129.0540$ (Figure 4). The major fragments at $\mathrm{m} / \mathrm{z} 493$ and 331 due to loss of rhamnose-rhamnose followed by loss of galactose were observed in the MS/MS spectrum of rhamnazin 3-rhamninoside (7) (Figure 4 and Figure S6). Unlike rutin (2), the MS/MS spectrum rhamnazin 3-rhamninoside (7) displayed the ion at $m / z 163.0599$ (Figure 4 and Figure $\mathrm{S} 6$ ), $\mathrm{C}_{6} \mathrm{H}_{11} \mathrm{O}_{5}{ }^{+}$, calcd for 163.0601 (mass difference of $1.22 \mathrm{ppm}$ ), which was likely to be a fragment of galactose, $\mathrm{C}_{6} \mathrm{H}_{11} \mathrm{O}_{5}{ }^{+}$. Flavonol glycosides $\mathbf{8 - 1 0}$ have galactose in their molecules; indeed, the MS/MS spectra of these compounds showed a fragment ion of galactose at $m / z 163$ (Figures S7-S9, Supplementary Materials). While glucose in rutin (2) does not have a fragment ion at $m / z$ 163, galactose in flavonol glycosides 8-10 shows the characteristic fragment ion at $m / z$ 163; therefore, the fragment ion at $m / z 163$ might be used for the identification of galactose in mass spectrometry-based analysis of glycosides or oligosaccharide chains attached to biomolecules (i.e., glycoproteins). 


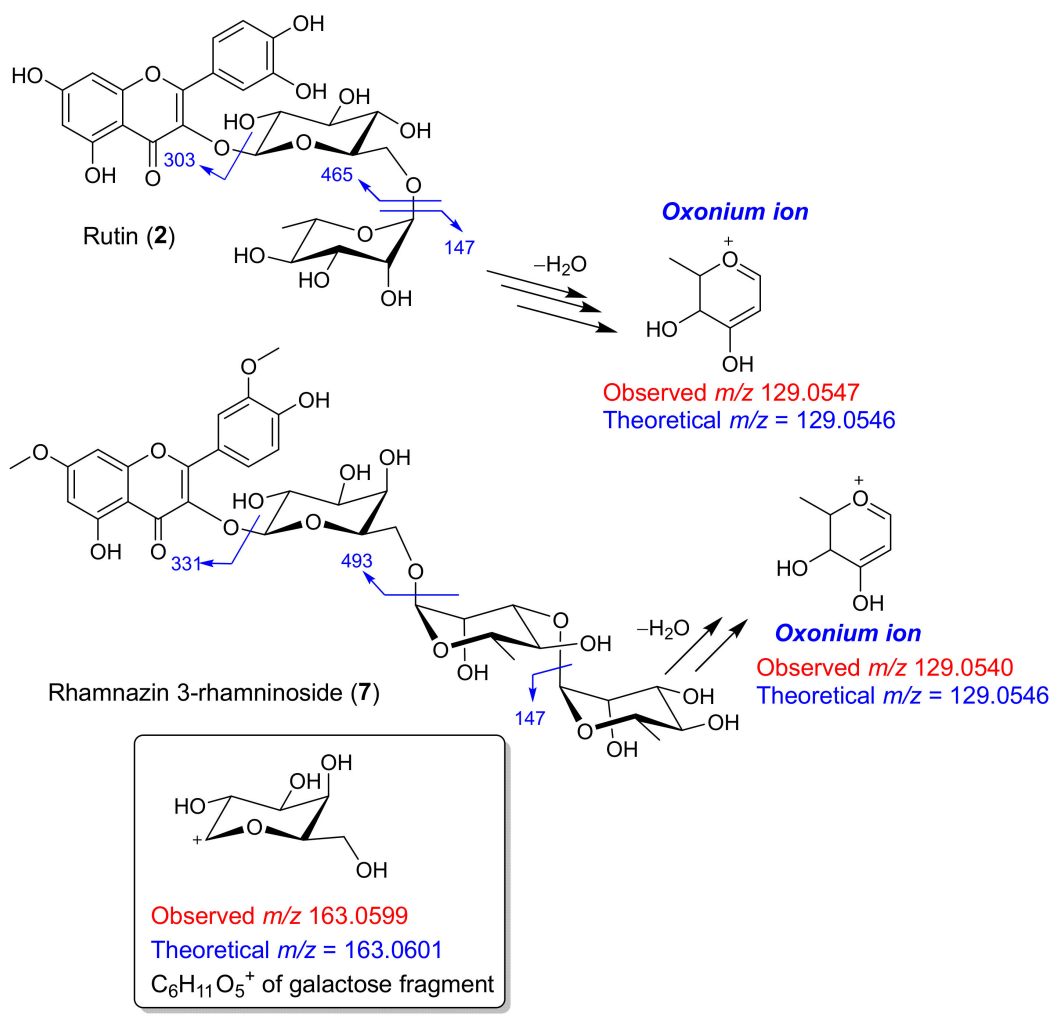

Figure 4. Typical MS fragmentation a standard compound, rutin (2), MS fragmentation of rhamnazin 3-rhamninoside (7) and an oxonium ion of rhamnose at $\mathrm{m} / \mathrm{z} 129.0$ and a galactose fragment ion at $m / z 163$.

In the present study, ventilatone B (12), a triterpene lupeol (13) and ventilatone A (15) (Figure 5) were also isolated from a $\mathrm{CH}_{2} \mathrm{Cl}_{2}$ extract of bark of $V$. denticulata. Ventilatones B (12) and A (15) are benzisochromanquinone, which were previously isolated from $V$. calyculata [36]. Structures of ventilatone B (12), lupeol (13) and ventilatone A (15) were characterized by analysis of NMR spectroscopy $\left({ }^{1} \mathrm{H},{ }^{13} \mathrm{C}\right.$ NMR and MS spectra are in Figures S27-S34, Supplementary Materials); their spectroscopic data were in good agreement with those reported in the literature [36,37]. Lupeol (13) was previously found in the plant genus Ventilago, for example, V. denticulata [38] and V. bombaiensis [39]. Note that rhamnalpinogenin (14) (Figure 5), which has the same molecular formula, $\mathrm{C}_{17} \mathrm{H}_{12} \mathrm{O}_{7}$, as that of ventilatone B (12), was tentatively identified by LC-MS/MS analysis, as revealed by both the Metlin Database and the Human Metabolome Database (Table 1, No. 70), observed at $\mathrm{m} / \mathrm{z} 329.0659$, calcd for $329.0656\left(\Delta_{m / z}=1.09 \mathrm{ppm}\right)$. However, there is a possibility that this putative compound is ventilatone B (12) because this benzisochromanquinone was previously isolated from $V$. calyculata [37], which is the same plant used in this work ( $V$. denticulata formerly known as $V$. calyculata). The MS/MS spectrum (Figure S25, Supplementary Materials) of the compound with the molecular formula $\mathrm{C}_{17} \mathrm{H}_{12} \mathrm{O}_{7}$ suggested that it is more likely to be ventilatone $\mathrm{B}$ (12) because of the loss of $\mathrm{C}_{2} \mathrm{HO}$, giving the fragment ion at $\mathrm{m} / \mathrm{z} 287.0551$ (Figure 5). In the case of rhamnalpinogenin (14), it should undergo a neutral loss of $\mathrm{CO}_{2}(44 \mathrm{amu})$ because it has a carboxylic group in its molecule (Figure 5) but none of the fragment ions were observed from the loss of $\mathrm{CO}_{2}$. Moreover, the molecular networking of ventilatone $B(\mathbf{1 2})$ is related to the compound with the $\mathrm{m} / \mathrm{z} 313.0706$ with a cosine similarity score of 0.84 (Figure 5). Analysis of MS/MS spectrum (Figure S26, Supplementary Materials) of the compound with the $m / z 313.0706$ revealed that this compound is likely to be ventilatone $\mathbf{A}(\mathbf{1 5})$, which undergoes the loss of $\mathrm{C}_{2} \mathrm{HO}$, giving the fragment ion at $\mathrm{m} / z 271.0604$ (Figure 5 ) that is analogous to ventilatone $\mathrm{B}(\mathbf{1 2})$. Note that the compound at the $\mathrm{m} / \mathrm{z} 313.0706$ was also listed in Table 1 (No. 61) and it was proposed to be 
aloe emodin w-acetate by Metlin Database and Human Metabolome Database. However, the MS/MS fragmentation suggested that this compound should be ventilatone A (15), not aloe emodin w-acetate.

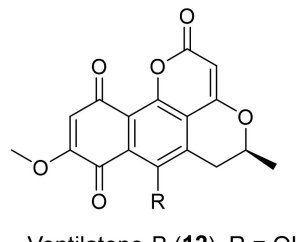

Ventilatone $\mathrm{B}(\mathbf{1 2}) \mathrm{R}=\mathrm{OH}$ Ventilatone $A(\mathbf{1 5}), R=H$
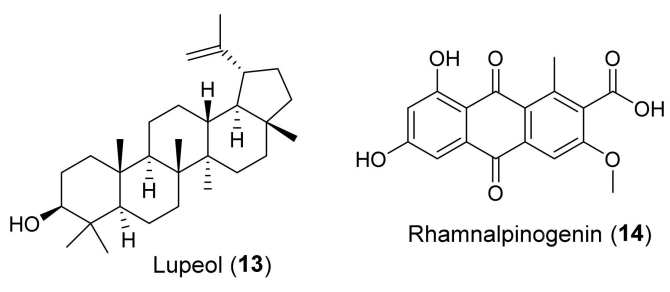

Rhamnalpinogenin (14)
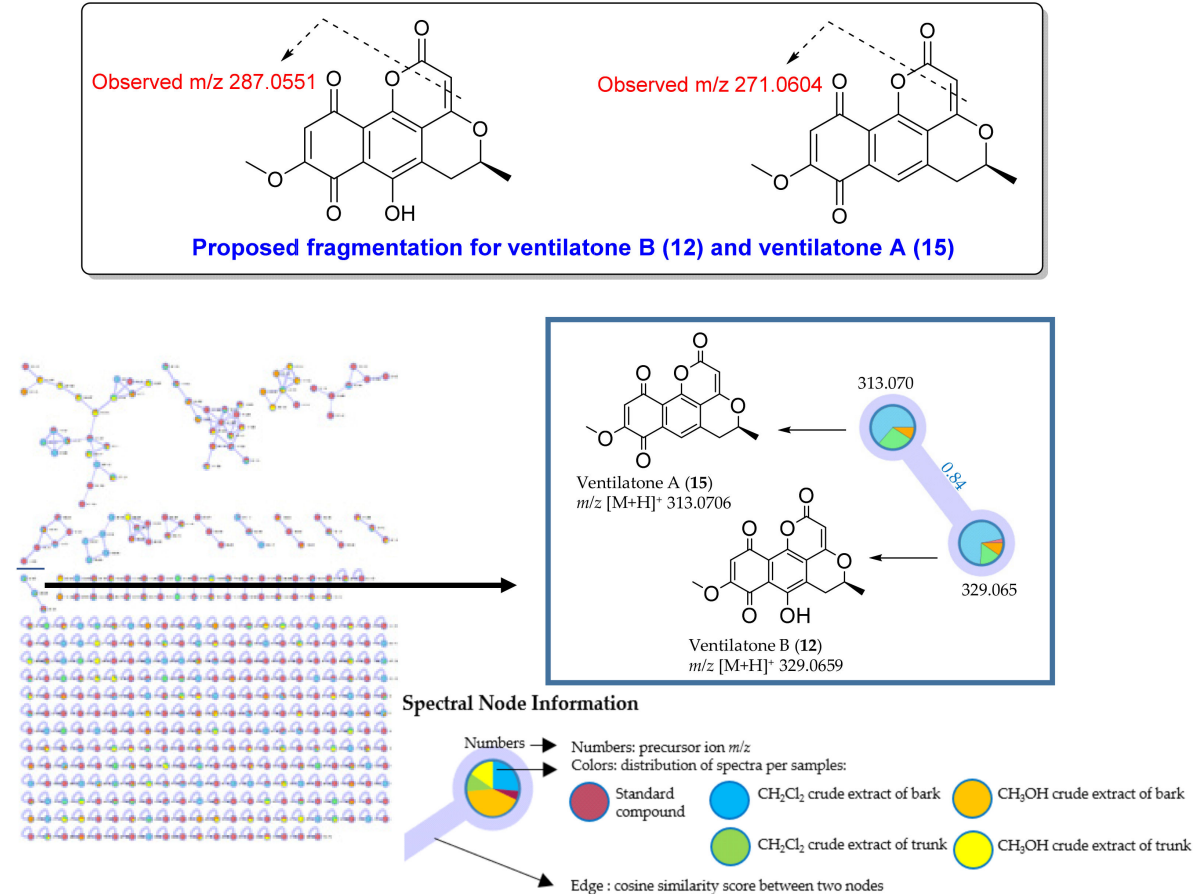

Figure 5. Structures of ventilatone B (12), lupeol (13), rhamnalpinogenin (14) and ventilatone A (15); proposed fragmentations and molecular networking of ventilatone B (12) and ventilatone A (15).

\subsection{Structure Elucidation of Ventilatone C (16)}

In the present work, a new compound-named ventilatone $\mathrm{C}(\mathbf{1 6})$-was isolated from a $\mathrm{CH}_{2} \mathrm{Cl}_{2}$ extract of a bark of $V$. denticulata (Figure 6). Structure elucidation of ventilatone C (16) was performed by analysis of NMR and MS data. Ventilatone $\mathrm{C}(\mathbf{1 6})$ was obtained as yellow amorphous solid and its molecular formula, $\mathrm{C}_{17} \mathrm{H}_{14} \mathrm{O}_{5}$, was obtained from ESI-HRMS, showing a pseudo-molecular ion at $\mathrm{m} / z 299.0917(\mathrm{M}+\mathrm{H})^{+}$, calcd for $\mathrm{C}_{17} \mathrm{H}_{15} \mathrm{O}_{5}, \mathrm{~m} / z$ 299.0919. ${ }^{1} \mathrm{H}$ and ${ }^{13} \mathrm{C}$ NMR spectra of ventilatone $\mathrm{C}$ (16) were similar to those of ventilatones $\mathrm{B}$ (12) and A (15), particularly on the signals for the fragment of 3-Me/H-3/H-4. ${ }^{1} \mathrm{H}$ NMR spectrum in $\mathrm{CDCl}_{3}$ of 16 showed signals of a hydroxyl proton at $\delta_{\mathrm{H}} 8.81(\mathrm{br} \mathrm{s})$, three aromatic protons at $\delta_{\mathrm{H}} 7.24(\mathrm{H}-5), 6.72(\mathrm{H}-6$ and $\mathrm{H}-8)$, one olefinic proton at $\delta_{\mathrm{H}} 5.74$, $\mathrm{sp}^{3}$ methine at $\delta_{\mathrm{H}} 4.53(\mathrm{H}-3)$, non-equivalent methylene at $\delta_{\mathrm{H}} 3.15$ and $3.00(\mathrm{H}-4)$ and two methyl groups at $\delta_{\mathrm{H}} 3.91$ (7-OMe) and 1.56 (3-Me) (Table 2). ${ }^{1} \mathrm{H}$ NMR signals in $\mathrm{CDCl}_{3}$ for $\mathrm{H}-6$ and $\mathrm{H}-8$ were overlapping at $\delta_{\mathrm{H}} 6.72$, however, these signals were clearly observed in acetone- $d_{6}$ as a doublet at $\delta_{\mathrm{H}} 6.94(\mathrm{H}-6)$ and $6.64(\mathrm{H}-8)$ and the $\mathrm{J}=2.3 \mathrm{~Hz}$ (Table 2) indicated the presence of meta coupling aromatic protons in $16 .{ }^{13} \mathrm{C}$ NMR and DEPT spectra of ventilatone $\mathrm{C}(\mathbf{1 6})$ showed seventeen signals attributable to two methyl, five methine, one methylene, nine quaternary carbons. ${ }^{1} \mathrm{H}-{ }^{1} \mathrm{H}$ COSY spectrum of 16 established the fragment of 3-Me/H-3/H-4 (as a bold line in Figure 6). HMBC spectrum of 16 showed the correlations from 3-Me to C-4; H-4 to C-4a; H-5 to C-4, C-5a, C-6, C-9a and C-10a; H-6 to C-5, C-7, C-8 and C-9a; H-8 to C-7 and C-9a; and H-13 to C-1, C10a and C-12 (Figure 6). The HMBC correlation 
from 7-OMe to $\mathrm{C}-7$ placed the methoxy group at the position $\mathrm{C}-7$, while that from 9-OH proton to $\mathrm{C}-8$, $\mathrm{C}-9$ and $\mathrm{C}-9 \mathrm{a}$ assigned the $\mathrm{OH}$ group at $\mathrm{C}-9$. Ventilatone $\mathrm{C}(\mathbf{1 6})$ had a positive optical rotation value $\left([\alpha]_{\mathrm{D}}^{25}+2.60\left(c 0.25, \mathrm{CHCl}_{3}\right)\right)$ similar to those of ventilatones $\mathrm{B}(\mathbf{1 2})\left([\alpha]_{\mathrm{D}}^{25}+30.62\left(c 0.5, \mathrm{CHCl}_{3}\right)\right.$ and $\mathrm{A}(\mathbf{1 5})$ $\left([\alpha]_{\mathrm{D}}^{25}+7.85\left(c 0.2, \mathrm{CHCl}_{3}\right)\right)$, both having $3 S$ stereochemistry, therefore, the $\mathrm{C}-3$ configuration in 16 was assigned to be $S$. Based on these spectroscopic data, the structure of ventilatone $C$ (16) was established as shown in Figure 6. Ventilatone C (16) has a structure closely related to pannorin B (17) [40] (Figure 6). However, pannorin B (17) was previously isolated from an endophytic fungus Penicillium sp. [40] and its biosynthetic pathway was proposed to be related to that of pannorin [41]. Interestingly, a fungal metabolite, pannorin B (17), shares the same chemical skeleton as that of ventilatones B (12) and A (15), which were isolated from tho nlant $V$ saliusulata $\Gamma 261$
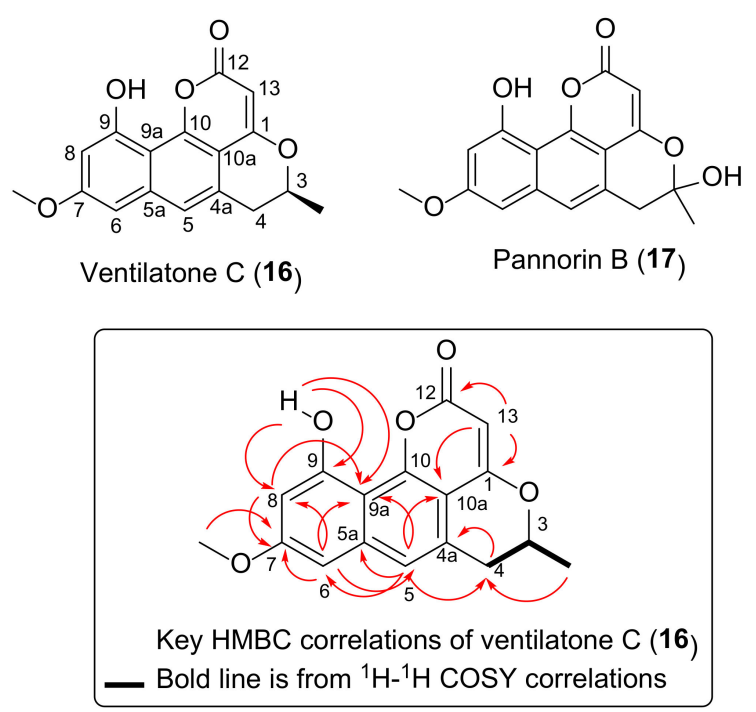

Figure 6. Structures of ventilatone $\mathrm{C}(\mathbf{1 6})$ and pannorin $\mathrm{B}(\mathbf{1 7})$, as well as key $\mathrm{HMBC}$ and ${ }^{1} \mathrm{H}-{ }^{1} \mathrm{H}$ COSY correlations of ventilatone $\mathrm{C}$ (16). HMBC correlations are from proton(s) to carbon.

Table 2. ${ }^{1} \mathrm{H}$ (400 MHz) and ${ }^{13} \mathrm{C}(100 \mathrm{MHz})$ NMR spectroscopic data for ventilatone C (16).

\begin{tabular}{|c|c|c|c|c|}
\hline \multirow{3}{*}{ Position } & \multicolumn{4}{|c|}{ Ventilatone C (16) } \\
\hline & \multicolumn{2}{|c|}{ NMR Data in $\mathrm{CDCl}_{3}$} & \multicolumn{2}{|c|}{ NMR Data in Acetone- $d_{6}$} \\
\hline & $\begin{array}{l}\delta_{\mathrm{H}}, \text { Multiplicity } \\
(\mathrm{J} \text { in Hz) }\end{array}$ & $\delta_{\mathrm{C}}$, Type & $\begin{array}{l}\delta_{\mathrm{H}}, \text { Multiplicity } \\
(J \text { in Hz) }\end{array}$ & $\delta_{\mathrm{C}}$, Type \\
\hline 1 & - & 166.18, C & - & 167.05, C \\
\hline 3 & $4.53, \mathrm{ddq}(10.5,6.3,3.4)$ & $74.97, \mathrm{CH}$ & $4.65, \mathrm{ddq}(10.6,6.3,3.4)$ & 76.06, $\mathrm{CH}$ \\
\hline 4 & $\begin{array}{c}3.00 \text {, ddd }(16.3,10.6,1.4) \\
3.15, \text { ddd }(16.4,3.3,0.8)\end{array}$ & $34.23, \mathrm{CH}_{2}$ & $\begin{array}{c}3.03, \text { ddd }(16.5,10.7,1.6) \\
3.25 \text {, ddd }(16.5,3.1,0.8)\end{array}$ & $34.50, \mathrm{CH}_{2}$ \\
\hline $4 a$ & - & 127.70, C & - & $129.66, \mathrm{C}$ \\
\hline 5 & $7.24, \mathrm{~s}$ & $120.60, \mathrm{CH}$ & $7.43, \mathrm{~s}$ & $121.32, \mathrm{CH}$ \\
\hline $5 a$ & - & $138.35, \mathrm{C}$ & - & $139.79, \mathrm{C}$ \\
\hline 6 & $6.72, \mathrm{~s}$ & $99.96, \mathrm{CH}$ & $6.94, \mathrm{~d}(2.3)$ & $100.52, \mathrm{CH}$ \\
\hline 7 & - & $161.13, \mathrm{C}$ & - & $161.07, \mathrm{C}$ \\
\hline 8 & $6.72, \mathrm{~s}$ & $103.11, \mathrm{CH}$ & 6.64, d (2.3) & $103.44, \mathrm{CH}$ \\
\hline 9 & - & $156.05, \mathrm{C}$ & - & $156.74, \mathrm{C}$ \\
\hline $9 a$ & - & $107.00, \mathrm{C}$ & - & $107.90, \mathrm{C}$ \\
\hline 10 & - & $152.72, \mathrm{C}$ & - & $153.48, \mathrm{C}$ \\
\hline $10 \mathrm{a}$ & - & $104.37, \mathrm{C}$ & - & 105.33, C \\
\hline 12 & - & $161.72, \mathrm{C}$ & - & $162.44, \mathrm{C}$ \\
\hline 13 & $5.74, \mathrm{~s}$ & $90.81, \mathrm{CH}$ & $5.63, \mathrm{~s}$ & $90.82, \mathrm{CH}$ \\
\hline 3-Me & $1.56, \mathrm{~d}(6.4)$ & $20.79, \mathrm{CH}_{3}$ & $1.54, \mathrm{~d}(6.3)$ & $20.83, \mathrm{CH}_{3}$ \\
\hline 7-OMe & $3.91, \mathrm{~s}$ & $55.50, \mathrm{CH}_{3}$ & $3.92, \mathrm{~s}$ & $55.88, \mathrm{CH}_{3}$ \\
\hline 9-OH & 8.81, br s & - & $8.94, \mathrm{~s}$ & - \\
\hline
\end{tabular}




\subsection{Antibacterial and Antifungal Activities of Crude Extracts, Fractions and Isolated Compounds}

As mentioned in the introduction part, local people in the West Midnapore district of West Bengal, the Eastern State of India, use the plant $V$. denticulata for the treatment of wound infection [15]. Bacteria found in wound infection were Staphylococcus aureus, Pseudomonas aeruginosa, Escherichia coli, Bacillus cereus and Salmonella enterica serovar Typhimurium [16-18], while the fungus Candida albicans was found in wound infection in diabetic foot ulcers [19]. Therefore, this research evaluated antibacterial and antifungal activities of crude extracts against S. aureus, P. aeruginosa, E. coli, B. cereus, S. enterica and C. albicans (Table 3). Methanol crude extracts of bark (MB) and trunk (MT) exhibited antibacterial activity against $B$. cereus, S. aureus, E. coli, S. enterica and P. aeruginosa with inhibition zones of 7-13 mm, 14-15 mm, $8 \mathrm{~mm}, 7-14 \mathrm{~mm}$ and 7-10 mm, respectively (Table 3). $\mathrm{A} \mathrm{CH}_{2} \mathrm{Cl}_{2}$ crude extract of bark (DB) displayed antibacterial activity against B. cereus, S. aureus, E. coli, S. enterica and P. aeruginosa with inhibition zones of $21 \mathrm{~mm}, 18 \mathrm{~mm}, 9 \mathrm{~mm}, 19 \mathrm{~mm}$ and $8 \mathrm{~mm}$, respectively (Table 3), while a $\mathrm{CH}_{2} \mathrm{Cl}_{2}$ crude extract of trunk (DT) showed antibacterial activity against $S$. aureus with inhibition zone of $13 \mathrm{~mm}$ (Table 3). Crude extracts, MB, DB and MT exhibited antifungal activity against C. albicans with inhibition zones of $13 \mathrm{~mm}, 16 \mathrm{~mm}$ and $8 \mathrm{~mm}$, respectively (Table 3). Fractions FM1-FM6 obtained from HPLC isolation of $\mathrm{MeOH}$ crude extract of bark were evaluated for antibacterial and antifungal activities. Fractions FM1-FM3 showed antibacterial activity toward the bacterial strains tested with inhibition zones of 8-14 mm, except that the fraction FM1 did not inhibit the growth of S. enterica (Table 3). Fraction FM4 exhibited the activity against $S$. enterica with inhibition zone of $14 \mathrm{~mm}$, while fraction FM6 displayed the activity toward bacteria E. coli and S. enterica with inhibition zone of $9 \mathrm{~mm}$ (Table 3). Fraction FM2 exhibited antifungal activity with inhibition zone of $10 \mathrm{~mm}$. Fractions FD1-FD6 from fractionation of a $\mathrm{CH}_{2} \mathrm{Cl}_{2}$ crude extract of bark displayed antibacterial activities toward the bacterial strains tested with inhibition zones of 9-30 mm, while the fractions FD1 and FD6 showed antifungal activity against $C$. albicans with inhibition zones of 17 and $9 \mathrm{~mm}$, respectively (Table 3).

Flavonoid glycosides 7-11 were isolated from MeOH crude extract of bark of $V$. denticulata and they were evaluated for antibacterial and antifungal activities (Table 3). Rhamnazin 3-rhamninoside (7) exhibited antibacterial activity against $S$. aureus with inhibition zone of $11 \mathrm{~mm}$, while catharticin or rhamnocitrin 3-rhamninoside (8) showed the activity toward B. cereus and E. coli with respective inhibition zones of $10 \mathrm{~mm}$ and $11 \mathrm{~mm}$ (Table 3). Xanthorhamnin B or rhamnetin 3-rhamninoside (9) displayed antibacterial activity against B. cereus, S. aureus and P. aeruginosa with respective inhibition zones of $9 \mathrm{~mm}, 9 \mathrm{~mm}$ and $13 \mathrm{~mm}$ (Table 3). Kaempferol 3-rhamninoside (10) and flavovilloside or quercetin 3-rhamninoside (11) exhibited antibacterial activity against B. cereus, S. aureus and E. coli with inhibition zones of $9 \mathrm{~mm}, 11-14 \mathrm{~mm}$ and 10-12 mm, respectively (Table 3) but they did not possess antifungal activity toward C. albicans. Rhamnazin 3-rhamninoside (7), rhamnocitrin 3-rhamninoside (8) and xanthorhamnin B or rhamnetin 3-rhamninoside (9) displayed antifungal activity against C. albicans with inhibition zones of $8 \mathrm{~mm}, 12 \mathrm{~mm}$ and $6 \mathrm{~mm}$, respectively (Table 3). To our knowledge, this is the first report on antibacterial and antifungal activities of flavonoid glycosides 7-11. Recently, xanthorhamnin B or rhamnetin 3-rhamninoside (9) was found to have antioxidative and radioprotective properties [42]. Rhamnazin 3-rhamninoside (7), rhamnocitrin 3-rhamninoside (8) and rhamnetin 3-rhamninoside (9) were reported to exhibit antioxidant and free radical-scavenging activities [43]. Glycoside derivatives of kaempferol were previously found to exhibit potent antibacterial activity against methicillin-resistant $S$. aureus and vancomycin-resistant enterococci [44]. Previously, kaempferol, an aglycone of 10, was found to exhibit antibacterial activity toward E. coli and it acted as DNA gyrase inhibitor [45], while quercetin, an aglycone of 11, exhibited antibacterial and antioxidant activities [46], targeting D-alanine:D-alanine ligase [47]. Interestingly, quercetin diacylglycoside derivatives displayed antibacterial activity by inhibition of DNA gyrase and topoisomerase IV [48]. 
Table 3. Antibacterial and antifungal activities of crude extracts, fractions and isolated compounds.

\begin{tabular}{|c|c|c|c|c|c|c|}
\hline \multirow{3}{*}{$\begin{array}{c}\text { Crude Extracts/ } \\
\text { Fractions/ } \\
\text { Compounds }\end{array}$} & \multicolumn{6}{|c|}{ Zone of Inhibition (mm) } \\
\hline & \multicolumn{6}{|c|}{ Bacteria/Fungus } \\
\hline & B. cereus & S. aureus & E. coli & S. enterica & P. aeruginosa & C. albicans \\
\hline $\mathrm{MB}^{\mathrm{a}}$ & 13 & 15 & 8 & 14 & 10 & 13 \\
\hline $\mathrm{DB}^{\mathrm{a}}$ & 21 & 18 & 9 & 19 & 8 & 16 \\
\hline $\mathrm{MT}^{\mathrm{a}}$ & 7 & 14 & 8 & 7 & 7 & 8 \\
\hline $\mathrm{DT}^{\mathrm{a}}$ & 0 & 13 & 0 & 0 & 0 & 0 \\
\hline FM1 b & 11 & 14 & 9 & 0 & 10 & 0 \\
\hline $\mathrm{FM} 2 \mathrm{~b}$ & 11 & 12 & 9 & 8 & 8 & 10 \\
\hline FM3 $b$ & 9 & 7 & 12 & 10 & 8 & 0 \\
\hline FM4 ${ }^{b}$ & 0 & 0 & 0 & 14 & 0 & 0 \\
\hline FM5 b & 0 & 0 & 0 & 0 & 0 & 0 \\
\hline FM $^{b}$ & 0 & 0 & 9 & 9 & 0 & 0 \\
\hline $\mathrm{FD} 1^{\mathrm{c}}$ & 18 & 15 & 14 & 18 & 11 & 17 \\
\hline $\mathrm{FD} 2^{\mathrm{c}}$ & 19 & 22 & 17 & 23 & 9 & 0 \\
\hline $\mathrm{FD}^{\mathrm{c}}$ & 26 & 25 & 25 & 30 & 12 & 0 \\
\hline $\mathrm{FD} 4^{\mathrm{c}}$ & 17 & 12 & 13 & 24 & 12 & 0 \\
\hline FD5 ${ }^{c}$ & 14 & 15 & 16 & 14 & 9 & 0 \\
\hline $\mathrm{FD}^{\mathrm{C}}$ & 11 & 17 & 11 & 16 & 10 & 9 \\
\hline 7 & 0 & 11 & 0 & 0 & 0 & 8 \\
\hline 8 & 10 & 0 & 11 & 0 & 0 & 12 \\
\hline 9 & 9 & 9 & 0 & 0 & 13 & 6 \\
\hline 10 & 9 & 11 & 12 & 0 & 0 & 0 \\
\hline 11 & 9 & 14 & 10 & 0 & 0 & 0 \\
\hline 12 & 11 & 11 & 0 & 18 & 0 & 12 \\
\hline 13 & ND & 7 & ND & ND & 0 & 0 \\
\hline 15 & 13 & 17 & ND & 18 & 0 & 0 \\
\hline 16 & 13 & 13 & ND & 14 & 0 & 0 \\
\hline Chloramphenicol $^{\mathrm{d}}$ & 44 & 37 & 50 & 50 & 28 & ND \\
\hline Tetracycline $\mathbf{d}^{\mathbf{d}}$ & 40 & 39 & 40 & 44 & 29 & ND \\
\hline Amphotericin B ${ }^{\mathbf{e}}$ & ND & ND & ND & ND & ND & 23 \\
\hline
\end{tabular}

${ }^{a} \mathrm{MB}: \mathrm{MeOH}$ crude extract of bark; DB: $\mathrm{CH}_{2} \mathrm{Cl}_{2}$ crude extract of bark; MT: MeOH crude extract of trunk; $\mathrm{DT}: \mathrm{CH}_{2} \mathrm{Cl}_{2}$ crude extract of trunk. ${ }^{\mathrm{b}}$ FM1-FM6: Fractions obtained from HPLC isolation of MeOH crude extract of bark eluted at retention times ( $t_{R}$ ) of 1.0-6.0 $\mathrm{min}$ (FM1), 6.0-8.5 $\mathrm{min}$ (FM2), 8.5-12.0 $\mathrm{min}$ (FM3), 12.0-20.0 $\mathrm{min}$ (FM4), 20.0-28.0 $\mathrm{min}$ (FM5) and 28.0-34.0 min (FM6), respectively. HPLC conditions are in the Section 3.9. ${ }^{c}$ FD1-FD6: HPLC fractions from $\mathrm{CH}_{2} \mathrm{Cl}_{2}$ crude extract of bark eluted at retention times ( $\mathrm{t}_{\mathrm{R}}$ ) of 1.0-5.5 $\mathrm{min}$ (FD1), 5.5-6.6 $\mathrm{min}$ (FD2), 6.6-7.1 min (FD3), 7.1-8.3 $\mathrm{min}$ (FD4), 8.3-9.5 $\mathrm{min}$ (FD5) and 9.5-13.0 min (FD6), respectively. HPLC conditions are in the Section 3.9. ${ }^{\mathrm{d}}$ Chloramphenicol and tetracycline are standard drugs for antibacterial activity. ${ }^{\mathrm{e}}$ Amphotericin B is a standard drug for antifungal activity. ND = Not determined.

Ventilatone B (12), lupeol (13), ventilatones A (15) and ventilatone $\mathrm{C}$ (16) isolated from a $\mathrm{CH}_{2} \mathrm{Cl}_{2}$ crude extract of bark of $V$. denticulata displayed antibacterial and antifungal activities (Table 3). Ventilatone B (12) exhibited antibacterial activity against B. cereus, S. aureus and S. enterica with inhibition zones of $11 \mathrm{~mm}, 11 \mathrm{~mm}$ and $18 \mathrm{~mm}$, respectively and it also showed antifungal activity against $C$. albicans with inhibition zone of $12 \mathrm{~mm}$ (Table 3). Lupeol (13) displayed antibacterial activity against $S$. aureus with inhibition zone of $7 \mathrm{~mm}$ (Table 3) but did not exhibit antifungal activity. This is the first report on antibacterial and antifungal activities of ventilatone B (12). Lupeol (13) was previously reported to exhibit antibacterial activity against human pathogenic bacteria [49]. Ventilatones A (15) exhibited antibacterial activity against B. cereus, S. aureus and S. enterica with inhibition zones of $13 \mathrm{~mm}$, $17 \mathrm{~mm}$ and $18 \mathrm{~mm}$, respectively, while ventilatone $C$ (16) displayed antibacterial activity against B. cereus, S. aureus and S. enterica with inhibition zones of $13 \mathrm{~mm}, 13 \mathrm{~mm}$ and $14 \mathrm{~mm}$, respectively (Table 3). 


\subsection{Dereplication of Antibacterial and Antifungal Constituents from HPLC Fractions of V. denticulata}

Fractions FM1-FM3 and FD1-FD4 from HPLC separation showed antibacterial and antifungal activities (Table 3); therefore, efforts have been made to identify the compounds in these HPLC fractions. Since the tentatively identified compounds (Table 1) in V. denticulata were obtained from LC-MS/MS analysis using Metlin Database and Human Metabolome Database, as well as standard compounds, we employed the accurate mass from ESI-HRMS to identify the compounds in fractions possessing antibacterial and antifungal activities. The ranges of mass difference $(\Delta)$ between the observed and calculated $\mathrm{m} / \mathrm{z}$ values for each compound were ca $0.55-2.42 \mathrm{ppm}$, which is less than $5 \mathrm{ppm}$ and thus giving the molecular formula of the compounds. ESI-HRMS analysis revealed that the fraction FM1 contained kaempferol, chrysoeriol, kaempferol 3-rhamninoside (10), isopimpinellin, 3-hydroxyphloretin, rhamnocitrin 3-rhamninoside (8), rhamnetin 3-rhamninoside (9) and rhamnazin 3-rhamninoside (7) (Table 4). Antibacterial and antifungal activities of flavonoid glycosides 7-10 are already presented in Table 3. Kaempferol was previously found to be an antibacterial agent $[45,50]$. Antibacterial activity of a flavonoid, chrysoeriol, was recently reported [51,52], while antibacterial and antifungal activities of isopimpinellin were already established [53]. Therefore, all compounds in the fraction FM1 have antibacterial activity, except 3-hydroxyphloretin. Fraction FM2 contained rhamnetin, luteolin and 3,5,7-trihydroxy-4' ,6-dimethoxyflavanone (Table 4), as revealed by ESI-HRMS analysis. Rhamnetin was previously found to have antifungal activity and it is a phytoalexin in plants [54], while luteolin was formerly found to exhibit antifungal activity [55]. Luteolin is a known antibacterial agent [56,57] and it is a lead compound for the synthesis of antibacterial derivatives [58]. 3,5,7-Trihydroxy-4',6-dimethoxyflavanone was formerly isolated from a plant, Prunus domestica [59] but it has never been evaluated for any biological activity. ESI-HRMS analysis showed that the fraction FM3 had emodin, rhamnocitrin and palmidin A (Table 4). Antibacterial activity of emodin was reported by our group [22] and emodin was previously found to inhibit growth of the bacterium Haemophilus parasuis, a causative agent of Glässer's disease and thus being a potential drug candidate for treating Glässer's disease [60]. Previously, antibacterial activity of rhamnocitrin was reported [61,62], while the activity of palmidin A has never been reported to date. Overall, eleven antibacterial compounds including flavonoid glycosides 7-10, kaempferol, chrysoeriol, isopimpinellin, rhamnetin, luteolin, emodin and rhamnocitrin are tentatively identified from the active fractions FM1-FM3, suggesting that the dereplication technique by LC-MS/MS analysis rapidly identifies antibacterial agents in extracts and fractions. In the present work and our previous report [22], antibacterial glycosides 7-10 and emodin were isolated from $V$. denticulata.

ESI-HRMS analysis for compounds in fractions FD1-FD4 obtained from HPLC separation was performed (Table 4). Fraction FD1 contained eriodyctiol, cartorimine, chrysoeriol, rhamnetin, 3-hydroxyphloretin, xanthotoxol glucoside and furocoumarinic acid glucoside (Table 4); among these compounds, chrysoeriol and rhamnetin were previously found to have antibacterial and antifungal activities [51,52,54]. ESI-HRMS analysis revealed that the fraction FD2 contained ventilagodenin A, physcion, rhamnocitrin, ventilatone A (15), 3',7-dihydroxy-4', 8-dimethoxyisoflavone, rhamnazin, 3,5,7-trihydroxy-4',6-dimethoxyflavanone and ventilatone B (12) (Table 4). Ventilagodenin A was found to be an antibacterial agent by our group [22], while ventilatones B (12) and A (15) were isolated in the present work; their antibacterial and antifungal activities are reported in Table 3 . Rhamnocitrin and rhamnazin were formerly found as antibacterial agents [61,62]. FD3 was found to contain afzelechin, (+)-(R)-ventilagolin and mukurozidiol (Table 4); among these compounds, (+)-(R)-ventilagolin (1) was found to be an antibacterial compound by our research group [22], whereas mukurozidiol or byakangelicin was previously reported to have antibacterial activity [63]. FD4 contained emodin, $6 \alpha$-hydroxymaackiain, 2',3,5-trihydroxy-5',7-dimethoxyflavanone and palmidin A (Table 4); however, only emodin was found to be an antibacterial agent [22,60]. Overall, ten antibacterial compounds including chrysoeriol, rhamnetin, ventilagodenin A, rhamnocitrin, rhamnazin, mukurozidiol, emodin, (+)-(R)-ventilagolin (1) and ventilatones B (12) and A (15) were identified from fractions FD1-FD4. 
Table 4. Antibacterial and antifungal agents from the HPLC fractions of $V$. denticulata, tentatively identified by ESI-HRMS analysis based on the putative compounds listed in Table 1.

\begin{tabular}{|c|c|}
\hline Fraction & Compounds in Fractions \\
\hline FM1 a & $\begin{array}{l}\left.\text { Kaempferol (285.0391 }[\mathrm{M}-\mathrm{H}]^{-}\right) \text {, chrysoeriol }\left(299.0589[\mathrm{M}-\mathrm{H}]^{-}\right) \text {, unidentified } \mathrm{C}_{13} \mathrm{H}_{20} \mathrm{~N}_{3} \mathrm{O}_{8} \mathrm{~S}(377.0851 \\
\left.[\mathrm{M}-\mathrm{H}]^{-}\right) \text {, kaempferol 3-rhamninoside }\left(739.2091[\mathrm{M}-\mathrm{H}]^{-}\right) \text {, isopimpinellin }\left(305.0657\left[\mathrm{M}+\mathrm{CH} \mathrm{H}_{3} \mathrm{COO}\right]^{-}\right) \text {, } \\
\text { 3-hydroxyphloretin }\left(335.0760[\mathrm{M}+\mathrm{HCOO}]^{-}\right) \text {, rhamnocitrin 3-rhamninoside }\left(377.0851[\mathrm{M}-\mathrm{H}]^{-}\right) \text {, } \\
\text { unidentified } \mathrm{C}_{37} \mathrm{H}_{32} \mathrm{~N}_{3} \mathrm{O}_{15}\left(757.1768[\mathrm{M}-\mathrm{H}]^{-}\right) \text {, rhamnetin 3-rhamninoside }\left(769.2162[\mathrm{M}-\mathrm{H}]^{-}\right) \text {, rhamnazin } \\
\text { 3-rhamninoside }\left(783.2310[\mathrm{M}-\mathrm{H}]^{-}\right)\end{array}$ \\
\hline $\mathrm{FM} 2^{\mathrm{a}}$ & $\begin{array}{l}\text { Rhamnetin }\left(315.0475[\mathrm{M}-\mathrm{H}]^{-}\right) \text {, luteolin }\left(285.0391[\mathrm{M}-\mathrm{H}]^{-}\right), 3,5,7 \text {-trihydroxy-4', 6-dimethoxyflavanone } \\
\left(377.0846\left[\mathrm{M}+\mathrm{CH}_{3} \mathrm{COO}^{-}\right)\right.\end{array}$ \\
\hline $\mathrm{FM}^{\mathrm{a}}$ & $\begin{array}{l}\text { Emodin }\left(269.0445[\mathrm{M}-\mathrm{H}]^{-}\right) \text {, rhamnocitrin }\left(299.0563[\mathrm{M}-\mathrm{H}]^{-}\right) \text {, palmidin A }\left(509.1218[\mathrm{M}-\mathrm{H}]^{-}\right) \\
\text {unidentified }\left(523.1351[\mathrm{M}-\mathrm{H}]^{-}\right)\end{array}$ \\
\hline $\mathrm{FD} 1^{\mathrm{b}}$ & $\begin{array}{l}\text { Eriodyctiol }\left(287.0554[\mathrm{M}-\mathrm{H}]^{-}\right) \text {, cartorimine }\left(289.0705[\mathrm{M}-\mathrm{H}]^{-}\right) \text {, chrysoeriol }\left(299.0558[\mathrm{M}-\mathrm{H}]^{-}\right) \text {, } \\
\text { rhamnetin }\left(315.0507[\mathrm{M}-\mathrm{H}]^{-}\right) \text {, 3-hydroxyphloretin }\left(335.0771[\mathrm{M}+\mathrm{HCOO}]^{-}\right) \text {, xanthotoxol glucoside } \\
\left(363.0709[\mathrm{M}-\mathrm{H}]^{-}\right) \text {, furocoumarinic acid glucoside }\left(365.0875[\mathrm{M}-\mathrm{H}]^{-}\right)\end{array}$ \\
\hline $\mathrm{FD} 2{ }^{\mathrm{b}}$ & $\begin{array}{l}\text { Ventilagodenin A }\left(275.0846[\mathrm{M}-\mathrm{H}]^{-}\right) \text {, physcion }\left(283.0650[\mathrm{M}-\mathrm{H}]^{-}\right) \text {, rhamnocitrin }\left(299.0613[\mathrm{M}-\mathrm{H}]^{-}\right) \text {, } \\
\text { ventilatone A }\left(311.0602[\mathrm{M}-\mathrm{H}]^{-}\right), 3^{\prime}, 7 \text {-dihydroxy-4 } 4^{\prime}, 8 \text {-dimethoxyisoflavone }\left(313.0761[\mathrm{M}-\mathrm{H}]^{-}\right) \text {, } \\
\text { rhamnazin }\left(329.0726[\mathrm{M}-\mathrm{H}]^{-}\right), 3,5,7-\text { trihydroxy-4 } 4^{\prime}, 6 \text {-dimethoxyflavanone }\left(331.0803[\mathrm{M}-\mathrm{H}]^{-}\right) \text {, ventilatone } \\
\text { B }\left(327.0556[\mathrm{M}-\mathrm{H}]^{-}\right) \text {, unidentified } \mathrm{C}_{17} \mathrm{H}_{18} \mathrm{O}_{8}\left(349.0965[\mathrm{M}-\mathrm{H}]^{-}\right)\end{array}$ \\
\hline FD3 ${ }^{b}$ & Afzelechin $\left(273.0727[\mathrm{M}-\mathrm{H}]^{-}\right),(+)-(R)$-ventilagolin $\left(331.0827[\mathrm{M}-\mathrm{H}]^{-}\right)$, mukurozidiol $\left(333.0968[\mathrm{M}-\mathrm{H}]^{-}\right)$ \\
\hline $\mathrm{FD} 4{ }^{\mathrm{b}}$ & $\begin{array}{l}\text { Emodin }\left(269.0450[\mathrm{M}-\mathrm{H}]^{-}\right), 6 \alpha \text {-hydroxymaackiain }\left(299.0550[\mathrm{M}-\mathrm{H}]^{-}\right) \\
2^{\prime}, 3,5 \text {-trihydroxy-5 } \\
\text { unidentified } \mathrm{C}_{15} \mathrm{H}_{11}, 7 \text {-dimethoxyflavanone }\left(331.0816[\mathrm{M}-\mathrm{H}]_{4}^{-}\right) \text {, palmidin } \mathrm{A}\left(509.1261[\mathrm{M}-\mathrm{H}]^{-}\right)\end{array}$ \\
\hline
\end{tabular}

${ }^{a}$ FM1-FM3 = Fractions obtained from HPLC isolation of MeOH crude extract of bark eluted at retention times $\left(\mathrm{t}_{\mathrm{R}}\right)$ of 1.0-6.0 $\mathrm{min}$ (FM1), 6.0-8.5 $\mathrm{min}$ (FM2) and 8.5-12.0 $\mathrm{min}$ (FM3), respectively. ${ }^{\mathrm{b}}$ FD1-FD4 = Fractions from HPLC isolation of $\mathrm{CH}_{2} \mathrm{Cl}_{2}$ crude extract of bark eluted at retention times ( $\mathrm{t}_{\mathrm{R}}$ ) of 1.0-5.5 $\mathrm{min}$ (FD1), 5.5-6.6 min (FD2), 6.6-7.1 $\mathrm{min}$ (FD3) and 7.1-8.3 min (FD4), respectively. HPLC conditions are in the Section 3.9.

\section{Materials and Methods}

\subsection{General Experimental Procedures}

UHPLC-MS/MS was carried out using Agilent 1290 infinity II connected to Agilent 6545 QTOF. HPLC column is ACE Excel $C_{18}$ AR $(100 \times 2.1 \mathrm{~mm}, 1.7 \mu \mathrm{m})$ column. MS data were processed using MassHunter data acquisition software. ESI-HRMS spectra were acquired from Bruker MicroTOF mass spectrometer processed using Bruker daltonics data analysis 3.3 software. HPLC was performed by Waters 1525 binary pump connected to a 2998 photodiode array detector. A semi-preparative column is SunFire $C_{18}(19 \times 250 \mathrm{~mm}, 5.0 \mu \mathrm{m})$; the HPLC chromatogram was processed by Empower 2 software. NMR spectra were obtained from Bruker Avance $400 \mathrm{MHz}$ NMR spectrometer, processed by TopSpin software. Sephadex LH-20 was packed for column chromatography. Specific optical rotation of compound 16 was obtained from a JASCO P-1020 polarimeter.

Methanol hypergrade LiChrosolv (LC-MS grade) and formic acid LiChropur (LC-MS grade) were used as the mobile phase for LC-MS analysis. Methanol- $d_{4}, \mathrm{CDCl}_{3}$, acetone- $d_{6}$ were used as solvents for NMR analysis.

\subsection{Plant Materials and Extraction of Plant}

The plant Ventilago denticulata was collected from Prachin Buri province, Thailand. It was characterized by Forest Herbarium, Bangkok, Thailand, in April 2019. V. denticulata (Voucher specimen number: CRI712) was deposited at Chulabhorn Research Institute (CRI), Thailand. Fresh trunks of $V$. denticulata were separated from their barks and then cut into small pieces (around $\pm 0.5 \mathrm{~cm}$ ). Fresh plant samples were sequentially with $\mathrm{MeOH}$ and $\mathrm{CH}_{2} \mathrm{Cl}_{2}$; this is because fresh samples contain water and $\mathrm{MeOH}$, a water-miscible solvent, was first used as a solvent. Trunk $(1.7 \mathrm{~kg})$ was macerated sequentially with $\mathrm{MeOH}(2 \times 1.5 \mathrm{~L})$ and $\mathrm{CH}_{2} \mathrm{Cl}_{2}(2 \times 1.5 \mathrm{~L})$ at room temperature for 2 days to give $34.66 \mathrm{~g}$ of $\mathrm{MeOH}$ crude extract of trunk and $15.03 \mathrm{~g}$ of $\mathrm{CH}_{2} \mathrm{Cl}_{2}$ crude extract of trunk. Bark $(0.5 \mathrm{~kg})$ was macerated sequentially with $\mathrm{MeOH}(2 \times 1.0 \mathrm{~L})$ and $\mathrm{CH}_{2} \mathrm{Cl}_{2}(2 \times 1.0 \mathrm{~L})$ at room temperature for 2 days to give $24.38 \mathrm{~g}$ of $\mathrm{MeOH}$ crude extract of bark and $1.24 \mathrm{~g}$ of $\mathrm{CH}_{2} \mathrm{Cl}_{2}$ crude extract of bark. All crude extracts were stored and kept in a freezer $\left(-18^{\circ} \mathrm{C}\right)$. 


\subsection{Crude Extract and Preparation of Standard Compounds for LC-MS/MS Analysis}

$1 \mathrm{mg}$ of each crude extract was dissolved in $1 \mathrm{~mL}$ of methanol to make a stock solution with a concentration of $1 \mathrm{mg} / \mathrm{mL} .100 \mu \mathrm{L}$ of each stock solution was diluted with $900 \mu \mathrm{L} \mathrm{MeOH}$ to obtain the final concentration of $100 \mu \mathrm{g} / \mathrm{mL}$. This solution was filtered through $0.22 \mu \mathrm{m}$ and transferred into 2 mL-LC vial.

Stock solutions of the following compounds (1 mg each), ((+)-R-ventilagolin, emodin, rutin, naringenin, 6-hydroxy flavone, chrysin and (+)-catechin) were dissolved in $1 \mathrm{~mL}$ of methanol. $100 \mu \mathrm{L}$ of each stock solution was diluted with $900 \mu \mathrm{L}$ methanol to obtain the final concentration of $100 \mu \mathrm{g} / \mathrm{mL}$. These solutions were filtered through $0.22 \mu \mathrm{m}$ filter and transferred into $2 \mathrm{~mL}-\mathrm{LC}$ vial.

\subsection{UHPLC-ESI-QTOF-MS/MS Conditions}

Crude extracts and standard compounds were analyzed by UHPLC connected to Q-TOF MS. UHPLC column was ACE Excel $C_{18}$ AR $(100 \times 2.1 \mathrm{~mm}, 1.7 \mu \mathrm{m})$ and a flow rate was $0.2 \mathrm{~mL} / \mathrm{min}$ with an injection volume of $0.5 \mu \mathrm{L}$. The gradient elution was performed using the following conditions: (i) linear gradient from $40 \% \mathrm{CH}_{3} \mathrm{CN}\left(0.1 \%\right.$ formic acid) in $\mathrm{H}_{2} \mathrm{O}\left(0.1 \%\right.$ formic acid) to $100 \% \mathrm{CH} \mathrm{CN}_{3}$ (0.1\% formic acid) for 0-25 min, (ii) isocratic elution of $100 \% \mathrm{CH}_{3} \mathrm{CN}(0.1 \%$ formic acid) for $5 \mathrm{~min}$ (at time of 25-30 min), (iii) a linear gradient from $100 \% \mathrm{CH}_{3} \mathrm{CN}(0.1 \%$ formic acid) to $40 \% \mathrm{CH} 3 \mathrm{CN}$ ( $0.1 \%$ formic acid) in $\mathrm{H}_{2} \mathrm{O}(0.1 \%$ formic acid) for $4 \mathrm{~min}$ (at time of 30-34 min) and (iv) equilibrium time by isocratic elution with $40 \% \mathrm{CH}_{3} \mathrm{CN}\left(0.1 \%\right.$ formic acid) in $\mathrm{H}_{2} \mathrm{O}(0.1 \%$ formic acid) for 6 min (at time of 34-40 $\mathrm{min})$. The total run time was $40 \mathrm{~min}$.

Dual AJS (Agilent Jet Stream) ESI was used as an ion source arranged with sheath gas flow of $12 \mathrm{~L} / \mathrm{min}$, capillary temperature at $325^{\circ} \mathrm{C}$, the gas flow rate of $10 \mathrm{~L} / \mathrm{min}$, sheath gas temperature of $250{ }^{\circ} \mathrm{C}$, sheath gas flow of $12 \mathrm{~L} / \mathrm{min}$, nebulizer of 45 psig, capillary voltage of $3.5 \mathrm{kV}$, fragmentor of $150 \mathrm{~V}$, skimmer of $65 \mathrm{~V}$ and nozzle voltage of $1 \mathrm{kV}$. MS relative threshold and MS absolute threshold were set to $0.010 \%$ and 100 , respectively.

LC-MS scan total ion chromatogram (TIC) and base peak chromatogram (BPC) with a scan range of $100-1100 \mathrm{~m} / \mathrm{z}$ and the analysis was performed in both positive and negative ionization modes. $\mathrm{MS}$ scan rate is 2 spectra per min. Auto-MS ${ }^{2}$ was performed using fixed collision energy at $20 \mathrm{keV}$, at which the most predominant $\mathrm{MS}^{1}$ ions are chosen for $\mathrm{MS}^{2}$ fragmentation. Auto-MS ${ }^{2}$ acquisition shows MS/MS data around 80-95\% of precursor ions. The MS/MS data were acquired with a scan rate of 3 spectra per second with MS/MS scan range at 100-1100 m/z. Isolation width MS/MS was set at medium ( $c a 4 \mathrm{amu}$ ). The maximum precursor was 3 per cycle. The MS/MS relative threshold was set to $0.01 \%$ and MS/MS absolute threshold was set to 5 .

The reference mass correction was performed and set as auto recalibration using a reference solution with minimal height of 1000 counts and the detection window of $100 \mathrm{~m} / \mathrm{z}$. The ions at $m / z 121.0509$ (purine) and $m / z 922.0098$ (HP-0921) were selected as standard ion peaks in a positive ion mode, while the ions at $m / z 112.9856$ (TFA anion) and $m / z 1033.9881$ (HP-0921 + TFA anion) were selected as standard ion peaks in a negative ion mode. In the auto MS/MS preferred/exclude table, these reference masses must be written as exclusion mass [64].

\subsection{Molecular Networking}

\subsubsection{Converting MS/MS Data}

All acquired MS/MS data was converted into MzXML format for further analysis in the GNPS website by ProteoWizard supported by NET Framework 3.5 SP1 using the following parameters [7]:

- 32-Bit was selected for binary encoding precision and zip compression was unchecked.

- $\quad$ Peak picking was set as a filter to make the output data become centroid.

- MS-Levels 1 and 2 should be checked. 


\subsubsection{Molecular Networking by GNPS (Global Natural Products Social Molecular Networking)}

FTP client, WinSCP, was used to upload the converted MS/MS data to the MzXML format using the host ost ccms-ftp01.ucsd.edu; these data to system were then transferred automatically to the GNPS system. The uploaded data were available in GNPS website readily for uploading data to create the molecular networking on the GNPS website (http://gnps.ucsd.edu).

In the basic option setting, precursor ion and fragment ion mass tolerance were set to 0.5 and 0.02 , respectively. The advanced network setting systems were set to minimum pairs cos of 0.7, network TopK of 10, maximum connected component size of 100, minimum matched fragment ions of 4 , the minimum cluster size of 2 [64].

For further analysis, the spectra were searched and matched toward GNP spectral library. They were set to the library search minimum matched of 4, search analog of "do search," score threshold of 0.7, maximum analog search mass difference of 100. Cosine similarity score that shows closer score to 1 indicates higher similarity matched with the library spectra or representing identical spectra, whereas the score closer to 0 indicates no similarity. The calculation of cosine similarity was considered based on fragment ions, precursor ions and peak intensities [64].

\subsubsection{Visualization of Molecular Networking Using Cytoscape}

The molecular networking data obtained from the GNPS system were imported to Cytoscape 3.7.2 to visualize and simplify molecular networking in one display. Cytoscape was used for analyzing the whole profile of metabolites in all crude extracts and correlation between standard compounds and their analogs [7].

3.6. Isolation of (+)-(R)-Ventilagolin (1), Flavonoid Glycosides (7-11), Ventilatone B (12), Lupeol (13), Ventilatone A (15) and Ventilatone C (16)

A MeOH crude extract of bark (10.23 g) was subjected to Sephadex LH-20 $(5 \times 55 \mathrm{~cm})$ column chromatography (CC), eluted with $\mathrm{MeOH}$ to give 65 fractions. Fraction 9 (271 mg) and fraction 10 (206 mg) containing flavonol glycosides and they were further purified using semi-preparative $\mathrm{C}_{18} \mathrm{HPLC}$ column (Sunfire $5 \mu \mathrm{m}, 19 \times 250 \mathrm{~mm}$ ). The gradient elution was performed using the following conditions: (i) isocratic elution of $30 \% \mathrm{MeOH} / \mathrm{H}_{2} \mathrm{O}$ for $0-10 \mathrm{~min}$, (ii) a linear gradient from $30 \% \mathrm{MeOH} / \mathrm{H}_{2} \mathrm{O}$ to $60 \% \mathrm{MeOH} / \mathrm{H}_{2} \mathrm{O}$ over $60 \mathrm{~min}$ (at time of $10-70 \mathrm{~min}$ ), (iii) a linear gradient from $60 \%$ $\mathrm{MeOH} / \mathrm{H}_{2} \mathrm{O}$ to $100 \% \mathrm{MeOH} / \mathrm{H}_{2} \mathrm{O}$ for $15 \mathrm{~min}$ (at time of $70-85 \mathrm{~min}$ ), (iv) a further linear gradient from $100 \% \mathrm{MeOH} / \mathrm{H}_{2} \mathrm{O}$ to $30 \% \mathrm{MeOH} / \mathrm{H}_{2} \mathrm{O}$ for $5 \mathrm{~min}$ (at time of $85-90 \mathrm{~min}$ ) and (v) an isocratic elution with $30 \% \mathrm{MeOH} / \mathrm{H}_{2} \mathrm{O}$ over $10 \mathrm{~min}$ (at time of $90-100 \mathrm{~min}$ ). The total run time was $100 \mathrm{~min}$. UV detector was set at $276 \mathrm{~nm}$ and a flow rate was $10 \mathrm{~mL} / \mathrm{min}$. The injection volume was $400 \mu \mathrm{L}$. This HPLC purification yielded quercetin 3-rhamninoside (11, $\left.\mathrm{t}_{\mathrm{R}} 41 \mathrm{~min}, 8.6 \mathrm{mg}\right)$, kaempferol 3-rhamninoside (10, $\mathrm{t}_{\mathrm{R}} 46 \mathrm{~min}$, $13.4 \mathrm{mg})$, rhamnetin 3-rhamninoside $\left(9, \mathrm{t}_{\mathrm{R}} 60 \mathrm{~min}, 25.5 \mathrm{mg}\right)$, rhamnocitrin 3-rhamninoside $\left(8 \mathrm{t}_{\mathrm{R}} 66 \mathrm{~min}\right.$, $18.2 \mathrm{mg})$, rhamnazin 3-rhamninoside $\left(7, \mathrm{t}_{\mathrm{R}} 70 \mathrm{~min}, 35.9 \mathrm{mg}\right)$.

A $\mathrm{CH}_{2} \mathrm{Cl}_{2}$ crude extract of bark $(782 \mathrm{mg})$ was subjected to Sephadex LH-20 $(2 \times 132 \mathrm{~cm}) \mathrm{CC}$, eluted with $\mathrm{MeOH}$ to give 33 fractions. Fraction 7 was identified as lupeol (13, $3.0 \mathrm{mg})$. Fraction 23 was identified as (+)-ventilatone B (12,3.7 mg). Fraction $12(58.9 \mathrm{mg})$ containing naphthalene derivatives was further purified by semi-preparative $\mathrm{C}_{18}$ HPLC using a reversed-phase column (Sunfire $5 \mu \mathrm{m}$, $19 \times 250 \mathrm{~mm}$ ). UV detector was set at $276 \mathrm{~nm}$ and a flow rate was $10 \mathrm{~mL} / \mathrm{min}$. The injection volume was $400 \mu \mathrm{L}$. The isocratic elution was performed using $60 \% \mathrm{MeOH} / \mathrm{H}_{2} \mathrm{O}$ at a flowrate of $10 \mathrm{~mL} / \mathrm{min}$ to give (+)-(R)-ventilagolin (1, $\left.\mathrm{t}_{\mathrm{R}} 9 \mathrm{~min}, 2.3 \mathrm{mg}\right)$. An insoluble part of fraction 12 was also identified as $(+)-(R)$-ventilagolin $(\mathbf{1}, 24.9 \mathrm{mg})$.

An insoluble $\mathrm{CH}_{2} \mathrm{Cl}_{2}$ crude extract of bark (189 mg) was purified by semi-preparative $\mathrm{C}_{18}$ HPLC (Sunfire $5 \mu \mathrm{m}, 19 \times 250 \mathrm{~mm}$ ), eluted with an isocratic elution with $70 \%$ of $\mathrm{CH}_{3} \mathrm{CN} / \mathrm{H}_{2} \mathrm{O}$ and a flow rate was $10 \mathrm{~mL} / \mathrm{min}$. This HPLC purification gave ventilatone $\mathrm{A}\left(\mathbf{1 5}, \mathrm{t}_{\mathrm{R}} 6 \mathrm{~min}, 4.1 \mathrm{mg}\right)$, ventilatone $\mathrm{B}$ $\left(\mathbf{1 2}, \mathrm{t}_{\mathrm{R}} 7 \mathrm{~min}, 10.5 \mathrm{mg}\right)$ and ventilatone $\mathrm{C}\left(\mathbf{1 6}, \mathrm{t}_{\mathrm{R}} 9 \mathrm{~min}, 5.0 \mathrm{mg}\right)$. 


\subsection{Spectroscopic Data of a New Compound, Ventilatone C (16)}

Yellow amorphous solid; $[\alpha]_{\mathrm{D}}^{25}+2.60$ ( c 0.25, $\mathrm{CHCl}_{3}$ ); UV (LC-UV, $\left.\mathrm{H}_{2} \mathrm{O}: \mathrm{CH}_{3} \mathrm{CN}, 30: 70\right) \lambda_{\max } 364.1$, 288.0 and $233.5 \mathrm{~nm}$; ESI-HRMS: $m / z 299.0917(\mathrm{M}+\mathrm{H})^{+}$, calcd $m / z 299.0919$ for $\mathrm{C}_{17} \mathrm{H}_{15} \mathrm{O}_{5} ;{ }^{1} \mathrm{H}$ and ${ }^{13} \mathrm{C}$ NMR spectroscopic data, see Table 2.

\subsection{Structure Elucidation of the Isolated Compounds}

Structures of isolated compounds 7-13, 15 and 16 were elucidated by analysis of spectroscopic data (1D and 2D NMR, UV and ESI-HRMS spectroscopic techniques). ${ }^{1} \mathrm{H}$ and ${ }^{13} \mathrm{C}$ NMR spectra of compounds 7-13 and 15, as well as $1 \mathrm{D}$ and 2D NMR of a new compound, ventilatone C (16), are in the Supplementary Materials.

\subsection{HPLC Fractionation of V. denticulata Extracts}

$100 \mathrm{mg}$ of $\mathrm{MeOH}$ crude extract of bark of $V$. denticulata was dissolved in $60 \% \mathrm{MeOH}$ and filtered through $0.45 \mu \mathrm{m}$ filter before HPLC fractionation. A semi-preparative HPLC column, SunFire $\mathrm{C}_{18}$ $(19 \times 250 \mathrm{~mm}, 5.0 \mu \mathrm{m})$, was used. A gradient elution was performed using the following conditions: (i) linear gradient from $40 \% \mathrm{CH}_{3} \mathrm{CN}\left(0.1 \%\right.$ formic acid) in $\mathrm{H}_{2} \mathrm{O}\left(0.1 \%\right.$ formic acid) to $100 \% \mathrm{CH}_{3} \mathrm{CN}$ ( $0.1 \%$ formic acid) for $0-25 \mathrm{~min}$, (ii) isocratic elution of $100 \% \mathrm{CH}_{3} \mathrm{CN}(0.1 \%$ formic acid) for $5 \mathrm{~min}$ (at time of $25-30 \mathrm{~min})$, (iii) a linear gradient from $100 \% \mathrm{CH}_{3} \mathrm{CN}\left(0.1 \%\right.$ formic acid) to $40 \% \mathrm{CH}_{3} \mathrm{CN}$ ( $0.1 \%$ formic acid) in $\mathrm{H}_{2} \mathrm{O}(0.1 \%$ formic acid) for $4 \mathrm{~min}$ (at time of 30-34 min and (iv) an isocratic elution with $40 \% \mathrm{CH}_{3} \mathrm{CN}\left(0.1 \%\right.$ formic acid) in $\mathrm{H}_{2} \mathrm{O}(0.1 \%$ formic acid) for $6 \mathrm{~min}$ (at time of 34-40 min). The total run time was $40 \mathrm{~min}$. The flow rate was $10 \mathrm{~mL} / \mathrm{min}$. The injection volume was $400 \mu \mathrm{L}$. The UV detector was set at wavelength of 200-400 nm, monitoring at $276 \mathrm{~nm}$. This process yielded fractions FM1-FM7, which were obtained from HPLC fractionation of a MeOH crude extract of bark eluted at retention times $\left(t_{R}\right)$ of 1.0-6.0 $\mathrm{min}$ (FM1), 6.0-8.5 $\mathrm{min}$ (FM2), 8.5-12.0 $\mathrm{min}$ (FM3), 12.0-20.0 min (FM4), 20.0-28.0 $\mathrm{min}$ (FM5) and 28.0-34.0 $\mathrm{min}$ (FM6), respectively. Weights of fractions FM1-FM6 were $23.9 \mathrm{mg}, 15.7 \mathrm{mg}, 13.8 \mathrm{mg}, 6.9 \mathrm{mg}, 6.6 \mathrm{mg}$ and $6.5 \mathrm{mg}$, respectively. The fractions FM1-FM6 were subsequently tested for antibacterial and antifungal activities and results are shown in Table 3.

Fractionation of $\mathrm{CH}_{2} \mathrm{Cl}_{2}$ crude extract $(100 \mathrm{mg}$ ) of bark of $V$. denticulata was carried out in the same manner as that of a MeOH crude extract, giving fractions FD1-FD10 with retention times $\left(t_{R}\right)$ of 1.0-5.5 $\mathrm{min}$ (FD1), 5.5-6.6 $\mathrm{min}$ (FD2), 6.6-7.1 $\mathrm{min}$ (FD3), 7.1-8.3 $\mathrm{min}$ (FD4), 8.3-9.5 $\mathrm{min}$ (FD5) and 9.5-13.0 min (FD6), respectively. Weights of fractions FD1-FD6 were $15.6 \mathrm{mg}, 14.6 \mathrm{mg}, 12.7 \mathrm{mg}, 8.4 \mathrm{mg}$, $6.7 \mathrm{mg}$ and $6.4 \mathrm{mg}$, respectively. Fractions FD1-FD6 were tested for antibacterial and antifungal activities and results are shown in Table 3.

\subsection{ESI-HRMS Analysis for the Identification of Compounds in HPLC Fractions}

The fractions FM1-FM3 and FD1-FD4 from HPLC separation showing antibacterial and antifungal activities were subsequently analyzed by ESI-HRMS. The compounds in these fractions were tentatively identified by ESI-HRMS analysis based on the putative compounds listed in Table 1. The parameters setting were capillary exit of $-110.0 \mathrm{~V}$, skimmer of $-35.0 \mathrm{~V}$, hexapol RF of $-110.0 \mathrm{~V}$, hexapol 1 of $-24 \mathrm{~V}$, set corrector fill of $63 \mathrm{~V}$, set pulsar pull of $405 \mathrm{~V}$, set pulsar push of $405 \mathrm{~V}$, set reflector of $1.3 \mathrm{kV}$, set flight tube of $9 \mathrm{kV}$, set detector TOF of $1.99 \mathrm{kV}$ and scan range 100-1000 m/z. Results are displayed in Table 4.

\subsection{In-Vitro Antibacterial and Antifungal Assays}

\subsubsection{Preparation of Bacteria and Fungi for Bioassay}

The bacterial strains used for an antibacterial assay were P. aeruginosa (TISTR No. 357), E. coli (TISTR No. 117), S. enterica serovar Typhimurium (TISTR No. 1470), S. aureus (TISTR No. 746) and B. cereus (TISTR No. 035). C. albicans (TISTR No. 5554) was the fungal strain used in an antifungal assay. All bacteria and C. albicans were purchased from the Thailand Institute of Scientific and Technological 
Research (Pathum Thani, Thailand). The stocks of bacteria and fungus were stored and kept in the freezer $\left(-20^{\circ} \mathrm{C}\right)$. Each of the bacterial and fungal strains was taken from the stocks and cultivated in a nutrient agar plate at temperature $37^{\circ} \mathrm{C}$ for $24 \mathrm{~h}$ for bacteria and $48 \mathrm{~h}$ for C. albicans. A single colony of bacteria and C. albicans was selected and transferred into $10 \mathrm{~mL} 0.85 \%$ normal saline. Suspension of bacteria and fungi were adjusted to make the same turbidity with a $0.5 \mathrm{McF}$ arland standard using spectrophotometer UV-Vis at wavelength of $600 \mathrm{~nm}$ [21].

\subsubsection{Disk Diffusion Method for Antibacterial and Antifungal Assays}

The disk diffusion method was performed to screen antibacterial and antifungal activities. Amphotericin B was used as a standard drug for the antifungal test. Tetracycline and chloramphenicol were used as standard drugs for antibacterial assay. $20 \%$ ethanol in DMSO was used as a negative control. Sample and standard solutions were prepared at a concentration of $10 \mathrm{mg} / 100 \mu \mathrm{L}$ in the solvent $(20 \%$ ethanol in DMSO). Each sample $(10 \mu \mathrm{L})$ was impregnated in a sterile disk (Whatman antibiotic assay disk, diameter $6 \mathrm{~mm}$ ) to give $1 \mathrm{mg} / 10 \mu \mathrm{L}$ as a final concentration of each disk. However, compounds 12, 13, 15 and 16 were tested at a concentration of $0.5 \mathrm{mg} / 10 \mu \mathrm{L}$ due to the limited amount of the compounds obtained. Bacterial or fungal solution ( $\mathrm{ca} 10^{8} \mathrm{CFU} / \mathrm{mL}$ ) was spread on a nutrient agar plate. Each disk was carefully placed on the plate containing bacteria or fungal solution and the plate was then incubated at $37^{\circ} \mathrm{C}$ for $24 \mathrm{~h}$. The diameter of a clear zone was measured as an indicator of inhibition toward bacteria or fungi [21]. Standard drugs for antibacterial activity were tetracycline and chloramphenicol and a standard drug for antifungal activity was amphotericin B; their activities are shown in Table 3.

\section{Conclusions}

Nine antibacterial and antifungal natural products in the plant, $V$. denticulata, were isolated using UHPLC-ESI-QTOF-MS/MS-Based molecular networking guided isolation and dereplication. Five antimicrobial flavonoid glycosides (7-11), two benzisochromanquinone, ventilatones B (12) and A (15), a new naphthopyrone ventilatone C (16) and a triterpene lupeol (13) were isolated from $V$. denticulata. Dereplication technique also tentatively identified antimicrobial compounds in $V$. denticulata, including kaempferol, chrysoeriol, isopimpinellin, rhamnetin, luteolin, emodin, rhamnocitrin, ventilagodenin $\mathrm{A}$, rhamnazin and mukurozidiol. The present work suggests that the molecular networking guided isolation and dereplication could assist the identification of antibacterial and antifungal agents in extracts of a plant. The presence of many antibacterial and antifungal compounds in the plant, $V$. denticulata, supports the traditional use of this plant as an herbal medicine for the treatment of wound infection.

Mass spectrometry-based molecular networking is a powerful dereplication strategy; it not only identifies known metabolites in complex mixtures but also suggests the presence of related analogues [6]. This work demonstrates that the molecular networking effectively assists the identification of antimicrobial compounds in plant extracts.

Supplementary Materials: The following supporting materials are available online at http://www.mdpi.com/20796382/9/9/606/s1 (Figures S1-S46); Overlay of TIC chromatograms of $\mathrm{MeOH}$ and $\mathrm{CH}_{2} \mathrm{Cl}_{2}$ crude extracts (Figure S1); molecular networking of crude extracts in a negative ionization mode (Figure S2); MS/MS spectra of compounds 1 and 2-8 (Figures S3-S9); ${ }^{1} \mathrm{H},{ }^{13} \mathrm{C}$ NMR and MS spectra of compounds 7-11 (Figures S10-S24); MS/MS spectra of ventilatone B (12) and ventilatone A (15) (Figures S25 and S26); ${ }^{1} \mathrm{H},{ }^{13} \mathrm{C}$ NMR and MS spectra of compounds 12, 13 and 15 (Figures S27-S34); and 1D and 2D NMR spectra, MS and UV spectra of ventilatone C (16) (Figures S35-S46).

Author Contributions: M.A.: Investigation, Formal analysis, Writing-original draft; P.P.: Formal analysis; T.T.: Formal analysis; C.M.: Supervision; S.R.: Supervision, Funding acquisition; P.K.: Conceptualization, Writing-original draft, Writing-review \& editing. All authors have read and agreed to the published version of the manuscript.

Funding: This research received no external funding.

Acknowledgments: This work is supported by the Center of Excellence on Environmental Health and Toxicology, Science \& Technology Postgraduate Education and Research Development Office (PERDO), Ministry of Education. 
M.A acknowledges Chulabhorn Graduate Institute and ASEAN Foundation Joint Post-graduate Scholarship Supported by Thailand International Cooperation Agency (TICA). The authors thank Poramet Nachalaem, the Scientific and Technological Instruments Center, Mae Fah Luang University, for UHPLC-MS/MS operation.

Conflicts of Interest: The authors declare no conflict of interest.

\section{References}

1. Over, B.; Wetzel, S.; Grütter, C.; Nakai, Y.; Renner, S.; Rauh, D.; Waldmann, H. Natural-product-derived fragments for fragment-based ligand discovery. Nat. Chem. 2013, 5, 21-28. [CrossRef] [PubMed]

2. Newman, D.J.; Cragg, G.M. Natural products as sources of new drugs from 1981 to 2014. J. Nat. Prod. 2016, 79, 629-661. [CrossRef] [PubMed]

3. Patridge, E.; Gareiss, P.; Kinch, M.S.; Hoyer, D. An analysis of FDA-approved drugs: Natural products and their derivatives. Drug Discov. Today 2016, 21, 204-207. [CrossRef]

4. Cragg, G.M.; Newman, D.J. Natural products: A continuing source of novel drug leads. Biochim. Biophys. Acta 2013, 1830, 3670-3695. [CrossRef]

5. Wibowo, M.; Forster, P.I.; Guymer, G.P.; Hofmann, A.; Davis, R.A. Using UHPLC-MS profiling for the discovery of new dihydro-beta-agarofurans from Australian Celastraceae plant extracts. Molecules 2019, $24,859$. [CrossRef] [PubMed]

6. Yang, J.Y.; Sanchez, L.M.; Rath, C.M.; Liu, X.; Boudreau, P.D.; Bruns, N.; Glukhov, E.; Wodtke, A.; de Felicio, R.; Fenner, A.; et al. Molecular networking as a dereplication strategy. J. Nat. Prod. 2013, 76, 1686-1699. [CrossRef]

7. Fox Ramos, A.E.; Evanno, L.; Poupon, E.; Champy, P.; Beniddir, M.A. Natural products targeting strategies involving molecular networking: Different manners, one goal. Nat. Prod. Rep. 2019, 36, 960-980. [CrossRef]

8. Zani, C.L.; Carroll, A.R. Database for rapid dereplication of known natural products using data from MS and fast NMR experiments. J. Nat. Prod. 2017, 80, 1758-1766. [CrossRef]

9. Mohimani, H.; Gurevich, A.; Shlemov, A.; Mikheenko, A.; Korobeynikov, A.; Cao, L.; Shcherbin, E.; Nothias, L.F.; Dorrestein, P.C.; Pevzner, P.A. Dereplication of microbial metabolites through database search of mass spectra. Nat. Commun. 2018, 9, 4035. [CrossRef]

10. Quinn, R.A.; Nothias, L.F.; Vining, O.; Meehan, M.; Esquenazi, E.; Dorrestein, P.C. Molecular networking as a drug discovery, drug metabolism, and precision medicine strategy. Trends Pharmacol. Sci. 2017, 38, 143-154. [CrossRef]

11. Wang, M.; Carver, J.J.; Phelan, V.V.; Sanchez, L.M.; Garg, N.; Peng, Y.; Nguyen, D.D.; Watrous, J.; Kapono, C.A.; Luzzatto-Knaan, T.; et al. Sharing and community curation of mass spectrometry data with global natural products social molecular networking. Nat. Biotechnol. 2016, 34, 828-837. [CrossRef] [PubMed]

12. Zang, Y.; Gong, Y.; Gong, J.; Liu, J.; Chen, C.; Gu, L.; Zhou, Y.; Wang, J.; Zhu, H.; Zhang, Y. Fungal polyketides with three distinctive ring skeletons from the fungus Penicillium canescens uncovered by OSMAC and molecular networking strategies. J. Org. Chem. 2020, 85, 4973-4980. [CrossRef]

13. Woo, S.; Kang, K.B.; Kim, J.; Sung, S.H. Molecular networking reveals the chemical diversity of selaginellin derivatives, natural phosphodiesterase-4 inhibitors from Selaginella tamariscina. J. Nat. Prod. 2019, 82, 1820-1830. [CrossRef] [PubMed]

14. Gross, M. The race against antibiotics resistance. Curr. Biol. 2019, 29, R859-R861. [CrossRef]

15. Kadchumsang, S.; Sirisa-Ard, P.; Sookkhee, S.; Chansakaow, S. Antibacterial and antioxidant activities of lanna medicinal plants used in mahoog formula. Int. J. Pharm. Pharm. Res. 2015, 7, 366-370.

16. Bessa, L.J.; Fazii, P.; Di Giulio, M.; Cellini, L. Bacterial isolates from infected wounds and their antibiotic susceptibility pattern: Some remarks about wound infection. Int. Wound J. 2015, 12, 47-52. [CrossRef]

17. Michelotti, F.; Bodansky, H.J. Bacillus cereus causing widespread necrotising skin infection in a diabetic person. Pract. Diabetes 2015, 32, 169-170a. [CrossRef]

18. Sfeir, M.; Youssef, P.; Mokhbat, J.E. Salmonella typhi sternal wound infection. Am. J. Infect. Control 2013, 41, e123-e124. [CrossRef]

19. Kalan, L.; Grice, E.A. Fungi in the wound microbiome. Adv. Wound Care (New Rochelle) 2018, 7, $247-255$. [CrossRef]

20. Tambe, A.B.; Dhage, O.L.; Bhalerao, P.B.; Bhalerao, S.S.; Thokal, S.H.; Shende, V.S.; Bansode, A.S.; Devhadrao, N.V. In-vitro anti-fungal activity and phytochemical screening of stem bark extracts from Ventilago denticulata. J. Drug Deliv. Ther. 2019, 9, 359-362. [CrossRef] 
21. Venkata, S.P.; Murali, M.C.; da Silva, J.A.T.; Raju, B.A.; Sravani, R. Screening the antimicrobial and antioxidant potential of Ventilago denticulata, Scolopia crenata and Rivea hypocrateriformis from maredumilli forest, india. Med. Aromat. Plant Sci. Biotechnol. 2012, 6, 58-62.

22. Molee, W.; Phanumartwiwath, A.; Kesornpun, C.; Sureram, S.; Ngamrojanavanich, N.; Ingkaninan, K.; Mahidol, C.; Ruchirawat, S.; Kittakoop, P. Naphthalene derivatives and quinones from Ventilago denticulata and their nitric oxide radical scavenging, antioxidant, cytotoxic, antibacterial, and phosphodiesterase inhibitory activities. Chem. Biodivers. 2018, 15, e1700537. [CrossRef] [PubMed]

23. Jang, J.Y.; Shin, H.; Lim, J.W.; Ahn, J.H.; Jo, Y.H.; Lee, K.Y.; Hwang, B.Y.; Jung, S.J.; Kang, S.Y.; Lee, M.K. Comparison of antibacterial activity and phenolic constituents of bark, lignum, leaves and fruit of Rhus verniciflua. PLoS ONE 2018, 13, e0200257. [CrossRef] [PubMed]

24. Ruan, J.; Yan, J.; Zheng, D.; Sun, F.; Wang, J.; Han, L.; Zhang, Y.; Wang, T. Comprehensive chemical profiling in the ethanol extract of Pluchea indica aerial parts by liquid chromatography/mass spectrometry analysis of its silica gel column chromatography fractions. Molecules 2019, 24, 2784. [CrossRef] [PubMed]

25. Kachlicki, P.; Piasecka, A.; Stobiecki, M.; Marczak, Ł. Structural characterization of flavonoid glycoconjugates and their derivatives with mass spectrometric techniques. Molecules 2016, 21, 1494. [CrossRef]

26. Medentsev, A.G.; Arinbasarova, A.Y.; Akimenko, V.K. Biosynthesis of naphthoquinone pigments by fungi of the genus Fusarium. Appl. Biochem. Microbiol. 2005, 41, 503-507. [CrossRef]

27. Adorisio, S.; Fierabracci, A.; Muscari, I.; Liberati, A.M.; Cannarile, L.; Thuy, T.T.; Sung, T.V.; Sohrab, H.; Hasan, C.M.; Ayroldi, E.; et al. Fusarubin and anhydrofusarubin isolated from a Cladosporium species inhibit cell growth in human cancer cell lines. Toxins 2019, 11, 503. [CrossRef]

28. Riess-Maurer, I.; Wagner, H. Struktur und synthese von flavonol-triosiden aus rhamnus-arten. Tetrahedron 1982, 38, 1269-1278. [CrossRef]

29. Dutton, G.G.S.; Merrifield, E.H.; Laffite, C.; Pratviel-Sosa, F.; Wylde, R. Comparative NMR study of rhamnobioses-applications. Org. Magn. Reson. 1982, 20, 154-158. [CrossRef]

30. Wang, J.; Kasai, R.; Sakimori, M.; Miyakoshi, M.; Tanaka, O.; Jia, M.-R.; Ling, Y.-K. Flavonol glycosides from the fruits of Rhamus leptophylla. Phytochemistry 1988, 27, 3995-3996. [CrossRef]

31. Riess-Maurer, I.; Wagner, H.; Lipták, A. Synthese und ${ }^{13} \mathrm{C}-\mathrm{NMR}-$ untersuchung von flavonol-3-O-(dirhamnosyl) -galactosiden der xanthorhamnin-, catharticin- und alaterin-reihe. Tetrahedron Lett. 1979, 20,3695-3698. [CrossRef]

32. Zheng, Y.F.; Qi, L.W.; Zhou, J.L.; Li, P. Structural characterization and identification of oleanane-type triterpene saponins in Glycyrrhiza uralensis Fischer by rapid-resolution liquid chromatography coupled with time-of-flight mass spectrometry. Rapid Commun. Mass Spectrom. 2010, 24, 3261-3270. [CrossRef] [PubMed]

33. Peterman, S.M.; Mulholland, J.J. A novel approach for identification and characterization of glycoproteins using a hybrid linear ion trap/FT-ICR mass spectrometer. J. Am. Soc. Mass Spectrom. 2006, 17, 168-179. [CrossRef] [PubMed]

34. Halim, A.; Westerlind, U.; Pett, C.; Schorlemer, M.; Rüetschi, U.; Brinkmalm, G.; Sihlbom, C.; Lengqvist, J.; Larson, G.; Nilsson, J. Assignment of saccharide identities through analysis of oxonium ion fragmentation profiles in LC-MS/MS of glycopeptides. J. Proteome Res. 2014, 13, 6024-6032. [CrossRef]

35. Rath, C.B.; Schirmeister, F.; Figl, R.; Seeberger, P.H.; Schaffer, C.; Kolarich, D. Flagellin glycoproteomics of the periodontitis associated pathogen Selenomonas sputigena reveals previously not described $O$-glycans and rhamnose fragment rearrangement occurring on the glycopeptides. Mol. Cell. Proteom. 2018, 17, 721-736. [CrossRef] [PubMed]

36. Hanumaiah, T.; Marshall, D.S.; Rao, B.K.; Rao, J.U.M.; Rao, K.V.J.; Thomson, R.H. Naphthoquinone-lactones and extended quinones from Ventilago calyculata. Phytochemistry 1985, 24, 2669-2672. [CrossRef]

37. Asha, R.; Gayathri Devi, V.; Abraham, A. Lupeol, a pentacyclic triterpenoid isolated from Vernonia cinerea attenuate selenite induced cataract formation in Sprague Dawley rat pups. Chem. Biol. Interact. 2016, 245, $20-29$. [CrossRef]

38. Pongjanta, A.; Pangjit, K.; Srichairatanakool, S. Antioxidant activity and cytotoxic effect of Ventilago denticulata Willd leaves extracts. J. Med. Assoc. Thai. 2016, 99 (Suppl. 1), S51-S57.

39. Pepalla, S.B.; Jammula, S.R.; Rao, K.V.J.; Thomson, R.H. Quinones and tetracosanolide in Ventilago bombaiensis. Phytochemistry 1992, 31, 2103-2104. [CrossRef]

40. Kaur, A.; Raja, H.A.; Deep, G.; Agarwal, R.; Oberlies, N.H. Pannorin B, a new naphthopyrone from an endophytic fungal isolate of Penicillium sp. Magn. Reson. Chem. 2016, 54, 164-167. [CrossRef] 
41. Vagstad, A.L.; Newman, A.G.; Storm, P.A.; Belecki, K.; Crawford, J.M.; Townsend, C.A. Combinatorial domain swaps provide insights into the rules of fungal polyketide synthase programming and the rational synthesis of non-native aromatic products. Angew. Chem. Int. Ed. Engl. 2013, 52, 1718-1721. [CrossRef] [PubMed]

42. Chaudhri, A.A.; Nadeem, M.; Rahman, A.U.; Alam, T.; Sajjad, W.; Hasan, F.; Badshah, M.; Khan, S.; Rehman, F.; Shah, A.A. Antioxidative and radioprotective properties of glycosylated flavonoid, xanthorhamnin from radio-resistant bacterium Bacillus indicus strain TMC-6. Curr. Microbiol. 2020, 77, 1245-1253. [CrossRef]

43. Ammar, R.B.; Bhouri, W.; Sghaier, M.B.; Boubaker, J.; Skandrani, I.; Neffati, A.; Bouhlel, I.; Kilani, S.; Mariotte, A.-M.; Chekir-Ghedira, L.; et al. Antioxidant and free radical-scavenging properties of three flavonoids isolated from the leaves of Rhamnus alaternus L. (Rhamnaceae): A structure-activity relationship study. Food Chem. 2009, 116, 258-264. [CrossRef]

44. Otsuka, N.; Liu, M.H.; Shiota, S.; Ogawa, W.; Kuroda, T.; Hatano, T.; Tsuchiya, T. Anti-methicillin resistant Staphylococcus aureus (MRSA) compounds isolated from Laurus nobilis. Biol. Pharm. Bull. 2008, 31, 1794-1797. [CrossRef] [PubMed]

45. Wu, T.; Zang, X.; He, M.; Pan, S.; Xu, X. Structure-activity relationship of flavonoids on their anti-Escherichia coli activity and inhibition of DNA gyrase. J. Agric. Food Chem. 2013, 61, 8185-8190. [CrossRef]

46. Ramos, F.A.; Takaishi, Y.; Shirotori, M.; Kawaguchi, Y.; Tsuchiya, K.; Shibata, H.; Higuti, T.; Tadokoro, T.; Takeuchi, M. Antibacterial and antioxidant activities of quercetin oxidation products from yellow onion (Allium cepa) skin. J. Agric. Food Chem. 2006, 54, 3551-3557. [CrossRef]

47. Wu, D.; Kong, Y.; Han, C.; Chen, J.; Hu, L.; Jiang, H.; Shen, X. D-Alanine:D-alanine ligase as a new target for the flavonoids quercetin and apigenin. Int. J. Antimicrob. Agents 2008, 32, 421-426. [CrossRef]

48. Hossion, A.M.L.; Zamami, Y.; Kandahary, R.K.; Tsuchiya, T.; Ogawa, W.; Iwado, A.; Sasaki, K. Quercetin diacylglycoside analogues showing dual inhibition of DNA gyrase and topoisomerase IV as novel antibacterial agents. J. Med. Chem. 2011, 54, 3686-3703. [CrossRef]

49. Obey, J.K.; von Wright, A.; Orjala, J.; Kauhanen, J.; Tikkanen-Kaukanen, C. Antimicrobial activity of Croton macrostachyus stem bark extracts against several human pathogenic bacteria. J. Pathog. 2016, 2016, 1453428. [CrossRef]

50. del Valle, P.; García-Armesto, M.R.; de Arriaga, D.; González-Donquiles, C.; Rodríguez-Fernández, P.; Rúa, J. Antimicrobial activity of kaempferol and resveratrol in binary combinations with parabens or propyl gallate against Enterococcus faecalis. Food Control 2016, 61, 213-220. [CrossRef]

51. Bashyal, P.; Parajuli, P.; Pandey, R.P.; Sohng, J.K. Microbial biosynthesis of antibacterial chrysoeriol in recombinant Escherichia coli and bioactivity assessment. Catalysts 2019, 9, 112. [CrossRef]

52. Nascimento, P.L.; Nascimento, T.C.; Ramos, N.S.; Silva, G.R.; Gomes, J.E.; Falcao, R.E.; Moreira, K.A.; Porto, A.L.; Silva, T.M. Quantification, antioxidant and antimicrobial activity of phenolics isolated from different extracts of Capsicum frutescens (Pimenta Malagueta). Molecules 2014, 19, 5434-5447. [CrossRef] [PubMed]

53. Mohammed, A.A. In-vitro antibacterial, antifungal, antibiofilm, and antioxidant potentials of isopimpinellin recovered from Citrullus colocynthis. Int. J. Pharm. Pharm. Sci. 2016, 8, 117-122.

54. Fawe, A.; Abou-Zaid, M.; Menzies, J.G.; Belanger, R.R. Silicon-mediated accumulation of flavonoid phytoalexins in cucumber. Phytopathology 1998, 88, 396-401. [CrossRef] [PubMed]

55. Báidez, A.G.; Gómez, P.; Del Río, J.A.; Ortuño, A. Antifungal capacity of major phenolic compounds of Olea europaea L. against Phytophthora megasperma Drechsler and Cylindrocarpon destructans (Zinssm.) Scholten. Physiol. Mol. Plant Pathol. 2006, 69, 224-229. [CrossRef]

56. Basile, A.; Giordano, S.; Lopez-Saez, J.A.; Cobianchi, R.C. Antibacterial activity of pure flavonoids isolated from mosses. Phytochemistry 1999, 52, 1479-1482. [CrossRef]

57. Mori, A.; Nishino, C.; Enoki, N.; Tawata, S. Antibacterial activity and mode of action of plant flavonoids against Proteus vulgaris and Staphylococcus aureus. Phytochemistry 1987, 26, 2231-2234. [CrossRef]

58. Lv, P.C.; Li, H.Q.; Xue, J.Y.; Shi, L.; Zhu, H.L. Synthesis and biological evaluation of novel luteolin derivatives as antibacterial agents. Eur. J. Med. Chem. 2009, 44, 908-914. [CrossRef]

59. Parmar, V.S.; Vardhan, A.; Nagarajan, G.R.; Jain, R. Dihydroflavonols from Prunus domestica. Phytochemistry 1992, 31, 2185-2186. [CrossRef] 
60. Li, L.; Song, X.; Yin, Z.; Jia, R.; Li, Z.; Zhou, X.; Zou, Y.; Li, L.; Yin, L.; Yue, G.; et al. The antibacterial activity and action mechanism of emodin from Polygonum cuspidatum against Haemophilus parasuis in vitro. Microbiol. Res. 2016, 186-187, 139-145. [CrossRef]

61. Martini, N.D.; Katerere, D.R.; Eloff, J.N. Biological activity of five antibacterial flavonoids from Combretum erythrophyllum (Combretaceae). J. Ethnopharmacol. 2004, 93, 207-212. [CrossRef] [PubMed]

62. Jiang, H.; Hu, J.R.; Zhan, W.Q.; Liu, X. Screening for fractions of Oxytropis falcata Bunge with antibacterial activity. Nat. Prod. Res. 2009, 23, 953-959. [CrossRef] [PubMed]

63. Kwon, Y.-S.; Kobayashi, A.; Kajiyama, S.-I.; Kawazu, K.; Kanzaki, H.; Kim, C.-M. Antimicrobial constituents of Angelica dahurica roots. Phytochemistry 1997, 44, 887-889. [CrossRef]

64. Dorrestein, P.; Wang, M.; Bandeira, N.; Truman, A.W.; Schmid, R.; Jarmusch, A.K.; Aksenov, A.A.; Ndlovu, N.; Tugizimana, F.; Keyzers, R.A.; et al. Reproducible molecular networking of untargeted mass spectrometry data using GNPS. Nat. Protoc. 2020, 15, 1954-1991. [CrossRef]

(C) 2020 by the authors. Licensee MDPI, Basel, Switzerland. This article is an open access article distributed under the terms and conditions of the Creative Commons Attribution (CC BY) license (http://creativecommons.org/licenses/by/4.0/). 